\title{
Qualitative Studies of Infant and Young Child Feeding in Lower-Income Countries: A Systematic Review and Synthesis of Dietary Patterns
}

\author{
Alessandra N. Bazzano ${ }^{1, *}$ (D), Aiko Kaji ${ }^{1}$, Erica Felker-Kantor ${ }^{1}$, Lydia A. Bazzano ${ }^{2}$ and \\ Kaitlin S. Potts ${ }^{1}$ \\ 1 Department of Global Community Health and Behavioral Sciences, Tulane University School of Public \\ Health and Tropical Medicine, New Orleans, LA 70112, USA; akaji@tulane.edu (A.K.); \\ efelkerk@tulane.edu (E.F.-K.); kstorck@tulane.edu (K.S.P.) \\ 2 Department of Epidemiology, Tulane University School of Public Health and Tropical Medicine, \\ New Orleans, LA 70112, USA; lbazzano@tulane.edu \\ * Correspondence: abazzano@tulane.edu; Tel.: +1-504-988-2338
}

Received: 7 August 2017; Accepted: 12 October 2017; Published: 18 October 2017

\begin{abstract}
Continued high rates of both under- and over-nutrition in low- and low-middle-income countries highlight the importance of understanding dietary practices such as early and exclusive breastfeeding, and dietary patterns such as timely, appropriate complementary feeding-these behaviors are rooted in complex cultural ecologies. A systematic review and synthesis of available qualitative research related to infant and young child dietary patterns and practices from the perspective of parents and families in low income settings is presented, with a focus on barriers and facilitators to achieving international recommendations. Data from both published and grey literature from 2006 to 2016 was included in the review. Quality assessment consisted of two phases (Critical Appraisal Skills Program (CASP) guidelines and assessment using GRADE-CERQual), followed by synthesis of the studies identified, and subsequent thematic analysis and interpretation. The findings indicated several categories of both barriers and facilitators, spanning individual and system level factors. The review informs efforts aimed at improving child health and nutrition, and represents the first such comprehensive review of the qualitative literature, uniquely suited to understanding complex behaviors leading to infant and young child dietary patterns.
\end{abstract}

Keywords: qualitative research; infant nutritional physiological phenomena; breast feeding; feeding behavior

\section{Introduction}

Early nutrition is crucially important for children to survive, grow and develop into healthy adults who can lead rewarding lives and productively contribute to their communities, and improving nutrition among young children has widely been recognized as an international priority [1]. Nonetheless, almost half of all childhood deaths (those under age 5) continue to be linked to nutritional causes [2]. In addition to morbidity and mortality, the impacts of poor nutrition, both in the form of under- and overnutrition, include strong, negative intergenerational health consequences for descendants [3]. Alongside maternal nutrition and intrauterine exposures [4], nutritionally-based caring behaviors of families, including breastfeeding and complementary feeding, form the basis of child nutrition. As a result, the potential impact of improved dietary practices on child nutrition has been extensively studied and subsequently promoted for potential to greatly improve health [5-8].

The United Nations Children's Fund (UNICEF) and the World Health Organization (WHO) concur that optimal nutrition practices for childhood include early initiation of breastfeeding, exclusive 
breastfeeding for the first 6 months of life, followed by the addition of nutritionally adequate, safe, and appropriate complementary foods with continuation of breastfeeding for 1 year and longer $[9,10]$. Markers of appropriate infant and young child feeding include these practices, as well as the timely introduction of solid and semi-solid foods, adequately frequent provision of daily meals, dietary diversity, and consumption of iron rich foods. In response to the need for simple, practical indicators of appropriate feeding practices in children aged 6-23 months, WHO published a set of population-level indicators that could be obtained from large-scale survey data on feeding practices in varied international settings [11].

However, characterizing feeding practices through quantitative methods such as large-scale surveys can be challenging, particularly because these practices constitute multidimensional and often interrelated behaviors rooted in family systems and socioeconomic conditions, and feeding patterns also change rapidly within short intervals of age $[12,13]$. Nonetheless, information on infant and young child feeding (IYCF) practices is required to improve nutrition and health during the first 2 years of life. Over the last decade, numerous studies on improvement of nutrition-related behaviors for infants and young children have relied on qualitative research methods, well-suited to exploring complex behaviors and their underlying psychosocial and cultural drivers, to investigate infant and young child feeding [14-18]. This body of research is likely to contain useful information to further the understanding of behavioral approaches to improve child nutrition. A comprehensive summary of qualitative data on feeding practices in this age group from lower-income settings, including a synthesis of barriers and facilitators to recommended practices, is currently lacking in the biomedical literature.

The primary objective of the proposed study was to systematically review qualitative literature related to family experiences (particularly parental ones) of infant and young child feeding in low-income countries, synthesizing information on the barriers and facilitators that may relate to interventions to impact nutrition, survival, growth and development. The results provide an overview of qualitative studies relating parental perspectives on infant and child dietary patterns, in the interest of providing insights for developing, improving, and scaling nutrition interventions.

\section{Materials and Methods}

This systematic review was registered with the International Prospective Register of Systematic Reviews (PROSPERO): registration number CRD42016035677. The review followed guidelines from the Enhancing Transparency in Reporting the Synthesis of Qualitative Research (ENTREQ) statement. Due to the exclusive focus on qualitative research, the review employed the ENTREQ guidelines [19] in lieu of the Preferred Reporting Items for Systematic Reviews and Meta-Analyses guidelines, which are more specific to the requirements of quantitative literature reviews [20]. The Supplementary Material to this article contains the full ENTREQ checklist.

Studies were only reviewed if their results were directly obtained from participants who were parents or close family members of an infant or young child (0-2 years of age) at the time of the study. Family members, for the purposes of this review, were defined to include mothers, fathers or other caregivers living in the child's household who routinely engage in infant or young child feeding. Infant and child feeding practices were defined as all actions taken to meet the physiological nutritional needs of children in this age group, including but not limited to: breast feeding; introduction of solid, semi-solid, and/or family foods (known as complementary feeding); and continued breastfeeding of children alongside provision of solid/semi-solid food.

\subsection{Inclusion and Exclusion Criteria}

Included studies used widely accepted qualitative data collection methods, with well-described methodology, including for example: interviews, focus groups, direct observation, and participatory action research. Included studies also needed to have provided a clear description of recognized qualitative data analysis methods (e.g., Grounded Theory, narrative analysis, content analysis, thematic analysis). 
Excluded studies were those for which it was difficult to extract qualitative data, e.g., mixed methods studies without clearly labeled data, or studies in settings where perceptions of parents or caregivers around infant and young child feeding could not be clearly identified, such as summaries or aggregated data. Commentaries, protocols, and systematic reviews were not included in the analysis. Additionally, as the focus was on research from resource limited settings, studies from countries other than those defined by the World Bank as low-income countries and lower-middle income countries (which have a Gross National Income per capita of less than \$4125) were excluded [21].

\subsection{Search Strategy}

The following electronic databases were considered to be the most relevant for the topic and were searched: MEDLINE (PubMed); Embase; Cumulative Index to Nursing and Allied Health Literature (CINAHL: EBSCOhost). A health sciences librarian was consulted in the development of the database searching strategy.

The initial search strategy was developed for MEDLINE and then adapted for the other databases. Medical Subject Headings were initially used followed by free-text terms using controlled vocabulary (see Appendix A for a detailed description of the search strategy). Results were restricted to English language publications from the last 10 years, due to potential difficulties in translating and interpreting foreign language qualitative data by native English-speaking reviewers, and to ensure that the review identifies literature relating to the most current infant and young child feeding practices. In addition, reference lists of included studies were manually searched to identify any additional studies that fit the inclusion criteria.

The included grey literature was initially identified through listing of relevant websites to search for organizations working in nutrition in lower-income countries (in consultation with experts working in the field who use and disseminate data through websites for related nutrition research). A custom search engine was created using Google Custom Search. Within the Custom Search Engine, the relevant websites were searched using search strategies adapted from those used in the databases to reflect relevant keywords related to qualitative studies of infant and young child feeding practices.

The review also included documents identified through a manual review of organizational reports and reports from relevant meetings related to nutrition of young children in low income countries. Results were similarly limited to publications in English from the last 10 years.

A flow diagram using PRISMA guidelines for reporting of systematic reviews is presented in Figure 1 in reporting of the selection process and results [20]. For organization of initial search results, Endnote reference management software (EndNoteX8, Clarivate Analytics, Boston, MA, USA) was used, and results of searches imported to the software. At the first stage, duplicates and irrelevant studies were removed. Two independent reviewers then screened study titles and abstracts for suitability against inclusion and exclusion criteria. The decision to include or exclude a study was required to be agreed on by both reviewers. If after consultation a decision was not reached by the two reviewers, a third reviewer made the final decision. 


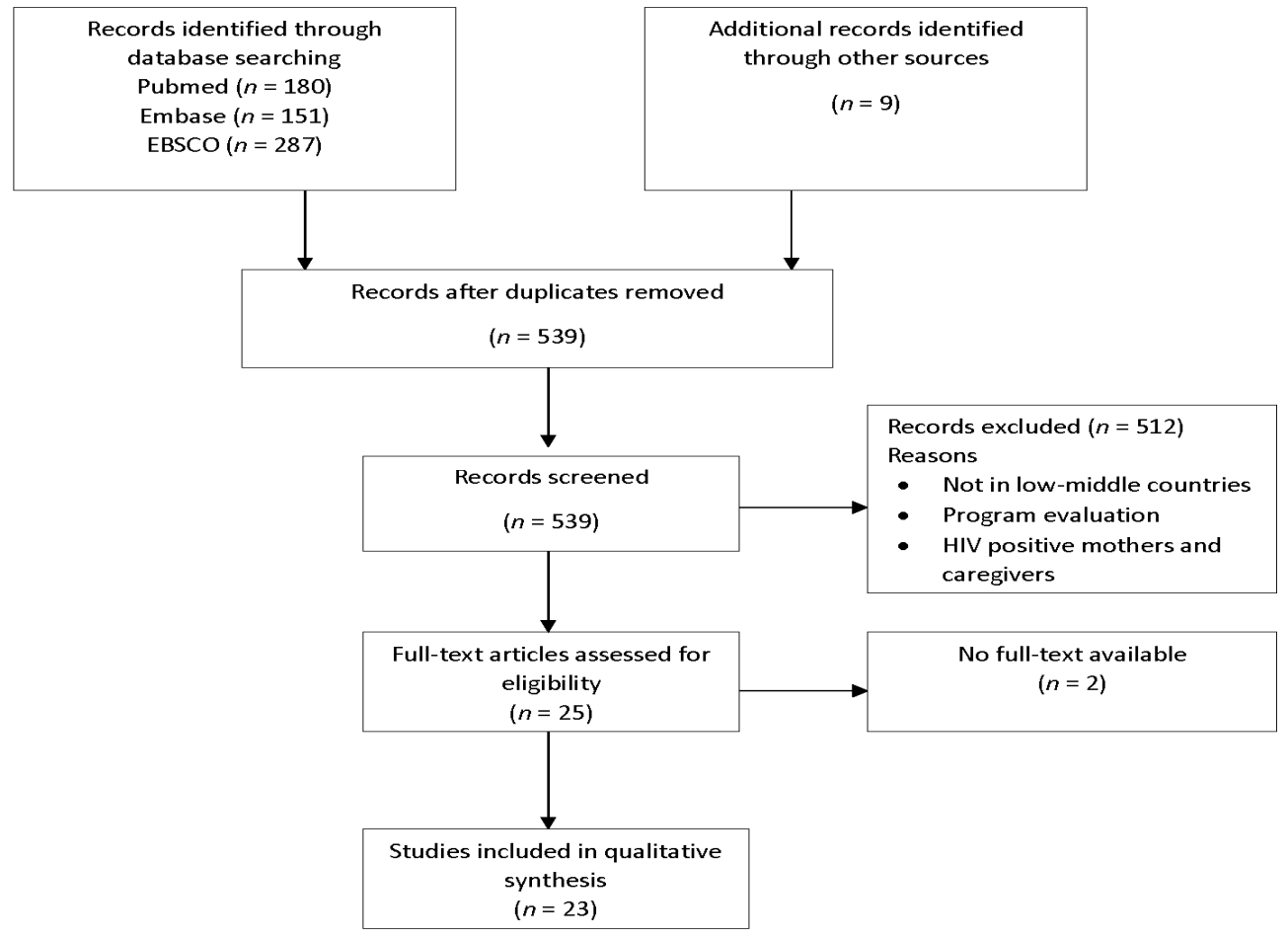

Figure 1. Flow diagram of search results and selection process.

\subsection{Data Extraction}

For organization of extracted data, a unified matrix was utilized to record specific characteristics of included studies. Extracted data included: reference details (author, year, title, journal/publisher); country/region of study; objectives or aims of the study; study design including methodological approaches (e.g., interviews/focus groups) and conceptual basis underlying the study (e.g., Grounded Theory); analysis method(s); sampling methodology and sample size; and initial assessment of the methodological limitations of the study. The initial results of the selection process and data abstraction are presented in Table 1.

Additional steps were taken in the data extraction phase that involved expanding the matrix (Table 1) to include participant characteristics, summaries of key outcomes/results reported, and the emergent review findings for which the study contributes evidence. These details are given in Appendix B. 
Table 1. Data extraction of studies included in review including bibliographic information, study details and assessment of methodological limitations.

\begin{tabular}{|c|c|c|c|c|c|c|c|}
\hline No. & $\begin{array}{l}\text { Author, Year, and } \\
\text { Country of Study }\end{array}$ & Title & Source Type & Aims of the Study & $\begin{array}{l}\text { Study Design, Analysis Method } \\
\text { (AM) }\end{array}$ & Sampling Method, Sample Size & $\begin{array}{l}\text { Assessment of Methodological } \\
\text { Limitations of Study (Numbers Refer } \\
\text { to CASP Questions) }\end{array}$ \\
\hline \multicolumn{8}{|c|}{ Studies Identified from Search Databases } \\
\hline 1 & $\begin{array}{l}\text { LM Babington } \\
\text { 2006 } \\
\text { Dominican Republic }\end{array}$ & $\begin{array}{l}\text { Understanding Beliefs, } \\
\text { Knowledge, and Practices of } \\
\text { Mothers in the Dominican } \\
\text { Republic Related to Feeding } \\
\text { Infants and Young Children }\end{array}$ & $\begin{array}{l}\text { Journal: } \\
\text { Hispanic } \\
\text { Health Care } \\
\text { International }\end{array}$ & $\begin{array}{l}\text { To expand knowledge regarding } \\
\text { feeding practices and nutritional } \\
\text { beliefs of mothers living in the } \\
\text { Dominican Republic. }\end{array}$ & $\begin{array}{l}\text { Qualitative study (focus } \\
\text { group discussion) } \\
\text { AM: Modified constant } \\
\text { comparative method }\end{array}$ & $\begin{array}{l}\text { Convenience sampling, } 10 \text { mothers aged } \\
16-45 \text { years with at least one child }\end{array}$ & $\begin{array}{l}\text { 5. no discussion of data } \\
\text { adequacy/saturation; limited } \\
\text { participants; ilimited explanation of } \\
\text { results; few illustrative quotes } \\
\text { 6. no discussion about the researcher's } \\
\text { role, potential bias and influence } \\
\text { 8. limited data to support the findings }\end{array}$ \\
\hline 2 & $\begin{array}{l}\text { A Omer-Salim, Persson, } \\
\text { P Olsson } \\
2007 \\
\text { Tanzania }\end{array}$ & $\begin{array}{l}\text { Whom can I rely on? } \\
\text { Mothers' approaches to } \\
\text { support for feeding: An } \\
\text { interview study in suburban } \\
\text { Dar es Salaam, Tanzania }\end{array}$ & $\begin{array}{l}\text { Journal: } \\
\text { Midwifery }\end{array}$ & $\begin{array}{l}\text { To explore and describe } \\
\text { mothers' perception of baby feeding } \\
\text { and approaches to support for baby } \\
\text { feeding \& to describe and explore } \\
\text { mothers' experiences of support for } \\
\text { baby feeding, with a focus on the } \\
\text { transition from exclusive to } \\
\text { non-exclusive breast feeding, in } \\
\text { suburban Dar es Salaam, Tanzania. }\end{array}$ & $\begin{array}{l}\text { Qualitative study } \\
\text { (semi-structured interviews) } \\
\text { AM: Qualitative content analysis }\end{array}$ & $\begin{array}{l}\text { Purposive sampling from a clinic, } 8 \\
\text { mothers with babies under } 6 \text { months of age }\end{array}$ & $\begin{array}{l}\text { 5. no discussion regarding saturation of } \\
\text { data; limited participants } \\
6 \text {. no discussion about the researcher's } \\
\text { role, potential bias and influence }\end{array}$ \\
\hline 3 & $\begin{array}{l}\text { C Tawiah-Agyemang, } \\
\text { BR Kirkwood, } \\
\text { K Edmond, A Bazzano, } \\
\text { Z Hill } \\
2008 \\
\text { Ghana } \\
\end{array}$ & $\begin{array}{l}\text { Early initiation of } \\
\text { breast-feeding in Ghana: } \\
\text { barriers and facilitators }\end{array}$ & $\begin{array}{l}\text { Journal: } \\
\text { Journal of } \\
\text { Perinatology }\end{array}$ & $\begin{array}{l}\text { To explore why women in Ghana } \\
\text { initiate breast-feeding early or late, } \\
\text { who gives advice about initiation, } \\
\text { and what foods or fluids are given to } \\
\text { babies when breast-feeding initiation } \\
\text { is late. }\end{array}$ & $\begin{array}{l}\text { Qualitative study } \\
\text { AM: Thematic analysis }\end{array}$ & $\begin{array}{l}\text { Purposive sampling, } 52 \text { semi-structured } \\
\text { interviews with recently delivered mothers } \\
8 \text { focus group discussions (FGD) with } \\
\text { women of child-bearing age }\end{array}$ & $\begin{array}{l}\text { 6. no discussion about the researcher's } \\
\text { role, potential bias and influence } \\
\text { 7. no explicit information about approval } \\
\text { from an ethics committee }\end{array}$ \\
\hline 4 & $\begin{array}{l}\text { GE Otoo, A. Lartey, } \\
\text { R Pérez-Escamilla } \\
2 \text { 2009 } \\
\text { Ghana }\end{array}$ & $\begin{array}{l}\text { Perceived Incentives and } \\
\text { Barriers to Exclusive } \\
\text { Breastfeeding Among } \\
\text { Peri-urban Ghanaian Women }\end{array}$ & $\begin{array}{l}\text { Journal: } \\
\text { Journal of } \\
\text { Human } \\
\text { Lactation }\end{array}$ & $\begin{array}{l}\text { To explore Ghanaian women's } \\
\text { knowledge and attitudes toward } \\
\text { exclusive breastfeeding }\end{array}$ & $\begin{array}{l}\text { Qualitative study (focus } \\
\text { group discussions) } \\
\text { AM: Thematic analysis }\end{array}$ & $\begin{array}{l}\text { Convenience sampling, } 4 \text { focus group } \\
\text { discussions with } 7 \text { to } 10 \text { mothers who had } \\
\text { at least one child less than } 4 \text { months }\end{array}$ & $\begin{array}{l}\text { 6. no discussion about the researcher's } \\
\text { role, potential bias and influence }\end{array}$ \\
\hline 5 & $\begin{array}{l}\text { L Hernandez, } \\
\text { ML Vasquez } \\
2010 \\
\text { Colombia }\end{array}$ & $\begin{array}{l}\text { Practices and beliefs about } \\
\text { exclusive breastffeeding by } \\
\text { women living in Commune } 5 \\
\text { in Cali, Colombia }\end{array}$ & $\begin{array}{l}\text { Journal: } \\
\text { Colombia } \\
\text { Médica }\end{array}$ & $\begin{array}{l}\text { To describe the practices and beliefs } \\
\text { about breastfeeding during the first } 6 \\
\text { months after delivery }\end{array}$ & $\begin{array}{l}\text { Qualitative study (Ethnonursing) } \\
\text { AM: Spradley's method; } \\
\text { (identification of domains, } \\
\text { taxonomies, componential } \\
\text { analysis, and finally themes) }\end{array}$ & $\begin{array}{l}\text { Purposive sampling, } 15 \text { key informant } \\
\text { participants (three interviews for each } \\
\text { participant: } 45 \text { interviews total); other } \\
\text { participants (relatives of the key } \\
\text { informant) }\end{array}$ & $\begin{array}{l}\text { 6. no discussion about the researcher's } \\
\text { role, potential bias and influence } \\
\text { 7. no explicit information about approval } \\
\text { from an ethics committee }\end{array}$ \\
\hline 6 & $\begin{array}{l}\text { S Rasheed, R Haider, } \\
\text { N Hassan, H Pachon, } \\
\text { S Islam, CS. Jalal, } \\
\text { TG Sanghvi } \\
2011 \\
\text { Bangladesh }\end{array}$ & $\begin{array}{l}\text { Why does nutrition } \\
\text { deteriorate rapidly among } \\
\text { children under } 2 \text { years of age? } \\
\text { Using qualitative methods to } \\
\text { understand community } \\
\text { perspectives on } \\
\text { complementary feeding } \\
\text { practices in Bangladesh }\end{array}$ & $\begin{array}{l}\text { Journal: Food } \\
\text { and Nutrition } \\
\text { Bulletin }\end{array}$ & $\begin{array}{l}\text { To understand community } \\
\text { perspectives on complementary } \\
\text { feeding practices in order to inform } \\
\text { the design of future interventions for } \\
\text { improved complementary feeding }\end{array}$ & $\begin{array}{l}\text { Mixed method study } \\
\text { (semi-structured interviews, food } \\
\text { attributes exercises, opportunistic } \\
\text { observations, trials of improved } \\
\text { practices, key informant } \\
\text { interviews, focus group } \\
\text { discussions) } \\
\text { AM: } \\
\text { Thematic analysis; data } \\
\text { triangulation of different data } \\
\text { sources identified themes to } \\
\text { understand phenomena }\end{array}$ & $\begin{array}{l}\text { Opportunistic sampling, } 42 \\
\text { semi-structured interviews with mothers; } 6 \\
\text { interviews with key informants; } 4 \text { focus } \\
\text { group discussions with } 2 \text { grandmothers } \\
\text { and fathers; food attribute exercises with } \\
24 \text { mothers; and } 21 \text { opportunistic } \\
\text { observations of feeding times involving } \\
\text { meals or snacks }\end{array}$ & $\begin{array}{l}\text { 6. no discussion about the researcher's } \\
\text { role, potential bias and influence }\end{array}$ \\
\hline 7 & $\begin{array}{l}\text { PC Lundberg, } \\
\text { TT Ngoc Thu } \\
2011 \text {. } \\
\text { Vietnam }\end{array}$ & $\begin{array}{l}\text { Breast-feeding attitudes and } \\
\text { practices among Vietnamese } \\
\text { mothers in Ho Chi Minh City }\end{array}$ & $\begin{array}{l}\text { Journal: } \\
\text { Midwifery }\end{array}$ & $\begin{array}{l}\text { To describe breast-feeding attitudes } \\
\text { and practices among Vietnamese } \\
\text { women in Ho Chi Minh City. }\end{array}$ & $\begin{array}{l}\text { Qualitative study } \\
\text { (semi-structured, in-depth } \\
\text { interviews and observation) } \\
\text { AM: Qualitative content analysis }\end{array}$ & $\begin{array}{l}\text { Purposive sampling, } 23 \text { mothers who had } \\
\text { given birth to a child within the last } \\
\text { two years }\end{array}$ & $\begin{array}{l}\text { 6. no discussion about the researcher's } \\
\text { role, potential bias and influence }\end{array}$ \\
\hline
\end{tabular}


Table 1. Cont.

\begin{tabular}{|c|c|c|c|c|c|c|c|}
\hline No. & $\begin{array}{l}\text { Author, Year, and } \\
\text { Country of Study }\end{array}$ & Title & Source Type & Aims of the Study & $\begin{array}{l}\text { Study Design, Analysis Method } \\
\text { (AM) }\end{array}$ & Sampling Method, Sample Size & $\begin{array}{c}\text { Assessment of Methodological } \\
\text { Limitations of Study (Numbers Refer } \\
\text { to CASP Questions) }\end{array}$ \\
\hline 8 & $\begin{array}{l}\text { Y Afiyanti, } \\
\text { D Juliastuti } \\
\text { 2012 } \\
\text { Indonesia } \\
\end{array}$ & $\begin{array}{l}\text { Exclusive breastfeeding } \\
\text { practice in Indonesia }\end{array}$ & $\begin{array}{l}\text { Journal: Birth } \\
\text { Journal of } \\
\text { Midwifery }\end{array}$ & $\begin{array}{l}\text { To explore cultural practices and } \\
\text { behaviors of breastfeeding among } \\
\text { Indonesian mothers }\end{array}$ & $\begin{array}{l}\text { Qualitative study } \\
\text { (semi-structured interviews) } \\
\text { AM: Grounded Theory, Constant } \\
\text { comparative analysis }\end{array}$ & $\begin{array}{l}\text { Purposive and theoretical sampling, } 8 \text { key } \\
\text { participants (breastfeeding mothers) } \\
10 \text { general participants (family members } \\
\text { and healthcare cadre) }\end{array}$ & $\begin{array}{l}\text { 5. no explanation regarding the approach } \\
\text { for research } \\
\text { 6. no discussion about the researcher's } \\
\text { role, potuntial bias and influence }\end{array}$ \\
\hline 9 & $\begin{array}{l}\text { HMH Lee, J Durham, } \\
\text { J Booth, V Sychareun } \\
2013 \\
\text { Lao PDR }\end{array}$ & $\begin{array}{l}\text { A qualitative study on the } \\
\text { breastfeeding experiences of } \\
\text { first-time mothers in } \\
\text { Vientiane, } \\
\text { Lao PDR }\end{array}$ & $\begin{array}{l}\text { Journal: BMC } \\
\text { Pregnancy and } \\
\text { Childbirth }\end{array}$ & $\begin{array}{l}\text { To identify the reasons and } \\
\text { influences for feeding decisions of } \\
\text { first-time mothers in Lao PDR. } \\
\text { Focusing on decisions around when } \\
\text { to initiate breast feeding, timing of } \\
\text { the introduction of complementary } \\
\text { foods and breast feeding duration. }\end{array}$ & $\begin{array}{l}\text { Qualitative study (interviews and } \\
\text { focus group discussions) } \\
\text { AM: Thematic analysis }\end{array}$ & $\begin{array}{l}\text { Purposive sampling, In-depth interviews } \\
\text { with16 first--ime mothers and } 7 \text { key } \\
\text { informants; focus group discussions with } \\
24 \text { first-time mothers }\end{array}$ & $\begin{array}{l}\text { 6. no discussion about the researcher's } \\
\text { role, potential bias and influence }\end{array}$ \\
\hline 10 & $\begin{array}{l}\text { C Bomer-Norton } \\
2013 \\
\text { Haiti }\end{array}$ & $\begin{array}{l}\text { Timing of breastfeeding } \\
\text { initiation in rural Haiti: } \mathrm{A} \\
\text { focused ethnography }\end{array}$ & $\begin{array}{l}\text { Dissertation: } \\
\text { The University } \\
\text { of Texas at } \\
\text { Arlington }\end{array}$ & $\begin{array}{l}\text { To describe factors affecting the } \\
\text { timing of breastfeeding initiation } \\
\text { among Haitian mothers, particularly } \\
\text { the specific cultural beliefs } \\
\text { and practices }\end{array}$ & $\begin{array}{l}\text { Qualitative study (ethnography) } \\
\text { AM: Ethnography }\end{array}$ & $\begin{array}{l}\text { Purposive sampling, } 25 \text { mothers with } \\
\text { children less than } 12 \text { months old }\end{array}$ & $\begin{array}{l}\text { 6. no discussion about the researcher's } \\
\text { role, potential bias and influence }\end{array}$ \\
\hline 11 & $\begin{array}{l}\text { A Laterra, MA Ayoya, } \\
\text { JM Beauliere, M Bienfait, } \\
\text { H Pachon } \\
2014 \\
\text { Haiti }\end{array}$ & $\begin{array}{l}\text { Infant and young child } \\
\text { feeding in four departments } \\
\text { in Haiti; mixed-method study } \\
\text { on prevalence of } \\
\text { recommended practices and } \\
\text { related attitudes, beliefs, and } \\
\text { other determinants }\end{array}$ & $\begin{array}{l}\text { Journal: Pan } \\
\text { American } \\
\text { Journal of } \\
\text { Public Health }\end{array}$ & $\begin{array}{l}\text { To determine and describe the } \\
\text { prevalence and patterns of three } \\
\text { recommended practices for infant } \\
\text { and young child feeding-exclusive } \\
\text { breastfeeding, continued } \\
\text { breastfeeding, and achievement of } \\
\text { minimum dietary diversity } \\
\text { To identify the attitudes and beliefs } \\
\text { that inform these practices and any } \\
\text { other factors that may facilitate or } \\
\text { impede their implementation }\end{array}$ & $\begin{array}{l}\text { Mixed method study (focus } \\
\text { group discussions) } \\
\text { AM: Thematic content analysis }\end{array}$ & $\begin{array}{l}\text { Convenience sampling of survey } \\
\text { respondents, } 12 \text { focus group discussions } \\
\text { among women } \geq 18 \text { years old with } \\
\text { children } \leq 2 \text { years old }\end{array}$ & $\begin{array}{l}\text { 5. no information regarding the format of } \\
\text { data collection (e.g., tape recordings, } \\
\text { notes etc.); no discussion about } \\
\text { saturation of data } \\
6 \text { 6. no discussion about the researcher's } \\
\text { role, potential bias and influence }\end{array}$ \\
\hline 12 & $\begin{array}{l}\text { G Pelto, } \\
\text { M Armar-Klemesu } \\
2015 \\
\text { Kenya }\end{array}$ & $\begin{array}{l}\text { Identifying interventions to } \\
\text { help rural Kenyan mothers } \\
\text { cope with food insecurity: } \\
\text { results of a focused } \\
\text { ethnographic study }\end{array}$ & $\begin{array}{l}\text { Journal: } \\
\text { Maternal and } \\
\text { Child } \\
\text { Nutrition }\end{array}$ & $\begin{array}{l}\text { To identify potential interventions to } \\
\text { improve the quality, availability, and } \\
\text { affordability of foods consumed by } \\
\text { infants and young children }\end{array}$ & $\begin{array}{l}\text { Qualitative study } \\
\text { (semi-structured interviews, } \\
\text { community observations, key } \\
\text { informants). } \\
\text { AM: Focused ethnographic study }\end{array}$ & $\begin{array}{l}\text { Random selection of } 32 \text { caregivers, } 8-10 \\
\text { key informants (no description of how } \\
\text { informants were selected). }\end{array}$ & $\begin{array}{l}\text { 5. no explanation regarding the sampling } \\
\text { or collection format of data; no } \\
\text { discussion about saturation of data } \\
\text { 6. no discussion about the researcher's } \\
\text { role, potential bias and influence }\end{array}$ \\
\hline 13 & $\begin{array}{l}\text { AN Bazzano, } \\
\text { RA Oberhelman, } \\
\text { KS Potts, LD Taub, } \\
\text { C Var } \\
2015 \\
\text { Cambodia }\end{array}$ & $\begin{array}{l}\text { What health service support } \\
\text { do families need for optimal } \\
\text { breastfeeding? An in-depth } \\
\text { exploration of young infant } \\
\text { feeding practices in } \\
\text { Cambodia }\end{array}$ & $\begin{array}{l}\text { Journal: } \\
\text { International } \\
\text { Journal of } \\
\text { Women's } \\
\text { Health }\end{array}$ & $\begin{array}{l}\text { To provide an in-depth } \\
\text { understanding of breastfeeding } \\
\text { practices in order to appropriately } \\
\text { design a scalable newborn health } \\
\text { intervention incorporating expanded } \\
\text { breastfeeding counseling and } \\
\text { support }\end{array}$ & $\begin{array}{l}\text { Qualitative study } \\
\text { (semi-structured interviews with } \\
\text { caregivers \& healthcare } \\
\text { providers) } \\
\text { AM: Formative research for } \\
\text { intervention development }\end{array}$ & $\begin{array}{l}\text { Purposive sampling, } 27 \text { interviews, } \\
14 \text { observations }\end{array}$ & $\begin{array}{l}\text { 5. no explanation regarding the approach } \\
\text { for research; no discussion about } \\
\text { saturation of data } \\
\text { 6. no discussion about the researcher's } \\
\text { role, potential bias and influence }\end{array}$ \\
\hline 14 & $\begin{array}{l}\text { J Burns, JA Emerson, } \\
\text { K Amundson, S Doocy, } \\
\text { LE Caulfield, } \\
\text { RD Klemm } \\
2016 \\
\text { Democratic Republic } \\
\text { of Congo }\end{array}$ & $\begin{array}{l}\text { A Qualitative Analysis of } \\
\text { Barriers and Facilitators to } \\
\text { Optimal Breastfeeding and } \\
\text { Complementary Feeding } \\
\text { Practices in South Kivu, } \\
\text { Democratic Republic } \\
\text { of Congo }\end{array}$ & $\begin{array}{l}\text { Journal: Food } \\
\text { and Nutrition } \\
\text { Bulletin }\end{array}$ & $\begin{array}{l}\text { To characterize IYCF practices and } \\
\text { barriers and enablers to optimal child } \\
\text { feeding in South Kivu }\end{array}$ & $\begin{array}{l}\text { Qualitative study (focus group } \\
\text { discussions, structured and } \\
\text { in-depth interviews) } \\
\text { AM: Thematic analysis }\end{array}$ & $\begin{array}{l}\text { Convenience sampling, } 188 \text { participants in } \\
20 \text { focus group discussions (mothers of } \\
\text { children < }<4 \text { months of age and women of } \\
\text { reproductive age), } 19 \text { structured } \\
\text { interviews (mothers with children }<24 \\
\text { months of age), } 43 \text { in-depth interviews } \\
\text { (lead mothers). }\end{array}$ & $\begin{array}{l}\text { 6. no discussion about the researcher's } \\
\text { role, potential bias and influence }\end{array}$ \\
\hline
\end{tabular}


Table 1. Cont.

\begin{tabular}{|c|c|c|c|c|c|c|c|}
\hline No. & $\begin{array}{l}\text { Author, Year, and } \\
\text { Country of Study }\end{array}$ & Title & Source Type & Aims of the Study & $\begin{array}{l}\text { Study Design, Analysis Method } \\
\text { (AM) }\end{array}$ & Sampling Method, Sample Size & $\begin{array}{c}\text { Assessment of Methodological } \\
\text { Limitations of Study (Numbers Refer } \\
\text { to CASP Questions) }\end{array}$ \\
\hline \multicolumn{8}{|c|}{ Grey Literature } \\
\hline 15 & $\begin{array}{l}\text { Alive and } \\
\text { Thrive-Ethiopia } \\
\text { 2010 } \\
\text { Ethiopia }\end{array}$ & $\begin{array}{l}\text { IYCF Practices, Beliefs, and } \\
\text { Influences in Tigray Region, } \\
\text { Ethiopia }\end{array}$ & $\begin{array}{l}\text { Alive and } \\
\text { Thrive }\end{array}$ & $\begin{array}{l}\text { To understand infant and young } \\
\text { child feeding practices and the role of } \\
\text { service providers in the } \\
\text { study communities. }\end{array}$ & $\begin{array}{l}\text { Qualitative study (focus group } \\
\text { discussion, interviews, } \\
\text { observations) } \\
\text { AM: Interpretative approach that } \\
\text { involved eliciting meanings from } \\
\text { the collected information }\end{array}$ & $\begin{array}{l}\text { Random selection of respondents from the } \\
\text { health post, and door to door sampling of } \\
\text { households with children in the required } \\
\text { age group; ; } 5 \text { interviews; FGD } \\
\text { (grandmothers and fathers) }\end{array}$ & $\begin{array}{l}\text { 6. no discussion about the researcher's } \\
\text { role, potential bias and influence } \\
\text { 8. lack of an in-depth description of the } \\
\text { analysis process }\end{array}$ \\
\hline 16 & $\begin{array}{l}\text { Alive and Thrive } \\
2010 \\
\text { Ethiopia }\end{array}$ & $\begin{array}{l}\text { IYCF Practices, Beliefs, and } \\
\text { Influences in SNNP Region, } \\
\text { Ethiopia }\end{array}$ & $\begin{array}{l}\text { Alive and } \\
\text { Thrive }\end{array}$ & $\begin{array}{l}\text { To understand infant and young } \\
\text { child feeding practices and the role of } \\
\text { service providers in the } \\
\text { study communities }\end{array}$ & $\begin{array}{l}\text { Qualitative study (focus group } \\
\text { discussion, interviews, } \\
\text { observations) } \\
\text { AM: Interpretative approach that } \\
\text { involved eliciting meanings from } \\
\text { the collected information }\end{array}$ & $\begin{array}{l}\text { Respondents were randomly selected in } \\
\text { each area from a list provided by the } \\
\text { health post, and the study interviewers } \\
\text { also went door to door to identify } \\
\text { households with children in the required } \\
\text { age group; } 47 \text { interviews (mothers); FGD } \\
\text { (grandmothers and fathers) }\end{array}$ & $\begin{array}{l}\text { 6. no discussion about the researcher's } \\
\text { role, potential bias and influence } \\
\text { 8. lack of an in-depth description of the } \\
\text { analysis process }\end{array}$ \\
\hline 17 & $\begin{array}{l}\text { United States Agency } \\
\text { for International } \\
\text { Development } \\
2010\end{array}$ & $\begin{array}{l}\text { Formative assessment of } \\
\text { infant and young child } \\
\text { feeding practices at the } \\
\text { community level in Zambia }\end{array}$ & $\begin{array}{l}\text { United States } \\
\text { Agency for } \\
\text { International } \\
\text { Development }\end{array}$ & $\begin{array}{l}\text { The objectives of this study were to: } \\
\text { describe the key influencers of infant } \\
\text { and young child feeding practices in } \\
\text { targeted areas in Zambiaia examine } \\
\text { the barriers and constraints to the } \\
\text { uptake of recommended feeding and } \\
\text { caring practices; learn more about the } \\
\text { types of foods used for } \\
\text { complementary feeding and the age } \\
\text { of children when these foods are } \\
\text { introduced; identify feasible and } \\
\text { effective channels for promoting } \\
\text { recommended infant and young } \\
\text { child feeding practices. }\end{array}$ & $\begin{array}{l}\text { Qualitative study (focus group } \\
\text { discussion and in-depth } \\
\text { interviews) } \\
\text { AM: Formative research, } \\
\text { Thematic analysis }\end{array}$ & $\begin{array}{l}\text { Targeted recruitment and purposive } \\
\text { sampling, } 24 \text { total FGDs with } 6 \text { FGDS in } 4 \\
\text { groups (mothers with children }<6 \text { months; } \\
\text { mothers with children } 6 \text { to } 23 \text { months of } \\
\text { age; fathers with children } \leq 23 \text { months; } \\
\text { grandmothers with children } \leq 23 \text { months }\end{array}$ & $\begin{array}{l}\text { 5. no explanation regarding the data } \\
\text { collection setting } \\
\text { 6. no discussion about the researcher's } \\
\text { role, potential bias and influence }\end{array}$ \\
\hline 18 & $\begin{array}{l}\text { United States Agency } \\
\text { for International } \\
\text { Development } \\
2011 \\
\text { Nigeria }\end{array}$ & $\begin{array}{l}\text { Formative assessment of } \\
\text { infant and young child } \\
\text { feeding practices-Federal } \\
\text { Capital Territory, Nigeria }\end{array}$ & $\begin{array}{l}\text { United States } \\
\text { Agency for } \\
\text { International } \\
\text { Development }\end{array}$ & $\begin{array}{l}\text { “(1) To conduct a cross-sectional } \\
\text { investigation of breastfeeding habits } \\
\text { of caregivers among target groups. } \\
\text { (2) To investigate complementary } \\
\text { feeding practices among } \\
\text { target troups. } \\
\text { (3) To document responsive feeding } \\
\text { practices, food hygiene, and feeding } \\
\text { the sick child practices in the } \\
\text { study areas. } \\
\text { (4) To identify prospects of health } \\
\text { communication interventions } \\
\text { through the use of relevant media for } \\
\text { reaching the respective target groups. } \\
\text { (5) To identify existing alternative } \\
\text { platforms for communication } \\
\text { interventions and information } \\
\text { sharing and dialogue among } \\
\text { target groups." }\end{array}$ & $\begin{array}{l}\text { Qualitative study (focus group } \\
\text { discussion with care givers) } \\
\text { AM: Formative research, } \\
\text { Thematic content analysis }\end{array}$ & $\begin{array}{l}\text { Purposive sampling (from health facility), } \\
\text { Focus group discussions ( } 91 \text { mothers of } \\
\text { children < } 6 \text { months old; } 81 \text { mothers of } \\
\text { children } 6 \text { to } 24 \text { months old } 75 \\
\text { grandmothers of childdren } \\
\text { under-24-month-olds; and } 82 \text { fathers of } \\
\text { children under-24- month-olds) }\end{array}$ & $\begin{array}{l}\text { 6. no discussion about the researcher's } \\
\text { role, potential bias and influence } \\
\text { 7. no information about approval from } \\
\text { the ethics committee }\end{array}$ \\
\hline
\end{tabular}


Table 1. Cont.

\begin{tabular}{|c|c|c|c|c|c|c|c|}
\hline No. & $\begin{array}{l}\text { Author, Year, and } \\
\text { Country of Study }\end{array}$ & Title & Source Type & Aims of the Study & $\begin{array}{l}\text { Study Design, Analysis Method } \\
\text { (AM) }\end{array}$ & Sampling Method, Sample Size & $\begin{array}{c}\text { Assessment of Methodological } \\
\text { Limitations of Study (Numbers Refer } \\
\text { to CASP Questions) }\end{array}$ \\
\hline 19 & $\begin{array}{l}\text { United States Agency } \\
\text { for International } \\
\text { Development } \\
2011 \\
\text { Malawi }\end{array}$ & $\begin{array}{l}\text { Consulting with caregivers } \\
\text {-formative research to } \\
\text { determine the barriers and } \\
\text { facilitators to optimal infant } \\
\text { and young child feeding in } \\
\text { three regions of Malawi }\end{array}$ & $\begin{array}{l}\text { United States } \\
\text { Agency for } \\
\text { International } \\
\text { Development }\end{array}$ & $\begin{array}{l}\text { Identify specific dietary problems } \\
\text { among children less than } 2 \text { years of } \\
\text { age, including determining the } \\
\text { energy density and nutrient density } \\
\text { (particularly iron, zinc, and vitamin } \\
\text { A) of common foods given to } \\
\text { children in different age groups. } \\
\text { Gain a further understanding of } \\
\text { current feeding practices and feeding } \\
\text { problems that Impede adequate } \\
\text { dietary intake in children less than } 2 \\
\text { years of age. } \\
\text { Describe maternal/child behaviors } \\
\text { related to responsive feeding. } \\
\text { Identify the positive and negative } \\
\text { social, cultural, and economic factors } \\
\text { that influence current practices, } \\
\text { including the influence of other } \\
\text { family and community members. }\end{array}$ & $\begin{array}{l}\text { Qualitative study (In-depth } \\
\text { interviews with care givers, } \\
\text { observations, and trials of } \\
\text { improved practices) } \\
\text { AM: Formative research, } \\
\text { Thematic content analysis }\end{array}$ & $\begin{array}{l}\text { Purposive sampling, Interviews with } 60 \\
\text { mothers/caregivers of children } 6-23 \\
\text { months and } 18 \text { key informants }\end{array}$ & $\begin{array}{l}\text { 6. no discussion about the researcher's } \\
\text { role, potential bias and influence }\end{array}$ \\
\hline 20 & $\begin{array}{l}\text { Academy for } \\
\text { Educational } \\
\text { Development } \\
\text { (AED)/Alive and Thrive } \\
\text { Ethiopia Country Office } \\
\text { 2011 } \\
\text { Ethiopia }\end{array}$ & $\begin{array}{l}\text { Initial Insight Mining and } \\
\text { Pretest Research for Alive and } \\
\text { Thrive Ethiopia }\end{array}$ & $\begin{array}{l}\text { Academy for } \\
\text { Educational } \\
\text { Development } \\
\text { (AED) / } \\
\text { Alive and } \\
\text { Thrive } \\
\text { Ethiopia } \\
\text { Country Office }\end{array}$ & $\begin{array}{l}\text { To assess IYCF practices as well as an } \\
\text { analysis of the specific information } \\
\text { gaps that needed to be explored } \\
\text { through the insight mining and } \\
\text { pretest research. }\end{array}$ & $\begin{array}{l}\text { Qualitative study (focus group } \\
\text { discussion with } \\
\text { mothers/caregivers) } \\
\text { AM: Not stated }\end{array}$ & $\begin{array}{l}\text { Multi-stage purposive sampling, } 32 \text { focus } \\
\text { group discussions among mothers, fathers } \\
\text { and mothers in law }\end{array}$ & $\begin{array}{l}\text { 5. no explanation of data collection } \\
\text { setting and the format of data } \\
\text { 6. no discussion about the researcher's } \\
\text { role, potential bias and influence } \\
\text { 7. no explicit information about approval } \\
\text { from the ethics committee }\end{array}$ \\
\hline 21 & $\begin{array}{l}\text { United States Agency } \\
\text { for International } \\
\text { Development } \\
2011 \\
\text { Kenya }\end{array}$ & $\begin{array}{l}\text { Engaging grandmothers and } \\
\text { men in infant and young } \\
\text { child feeing and maternal } \\
\text { nutrition-Report of a } \\
\text { formative assessment in } \\
\text { Eastern and Western Kenya }\end{array}$ & $\begin{array}{l}\text { United States } \\
\text { Agency for } \\
\text { International } \\
\text { Development }\end{array}$ & $\begin{array}{l}\text { Assess complementary feeding } \\
\text { practices of children younger than } 2 \\
\text { years and community beliefs that } \\
\text { influence these. } \\
\text { Determine maternal dietary practices } \\
\text { during pregnancy and lactation and } \\
\text { household factors that } \\
\text { influence these. } \\
\text { Establish the roles and } \\
\text { responsibilities of men and } \\
\text { grandmothers in the family and in } \\
\text { maternal dietary and infant and } \\
\text { young child feeding practices. } \\
\text { Determine cultural influencers of } \\
\text { maternal, infant, and young } \\
\text { child feeding. } \\
\text { Document available services and } \\
\text { support for nutrition in the } \\
\text { intervention areas. }\end{array}$ & $\begin{array}{l}\text { Qualitative study (focus group } \\
\text { discussion with } \\
\text { mothers/caregivers) } \\
\text { AM: Formative research, } \\
\text { Thematic content analysis }\end{array}$ & $\begin{array}{l}\text { Purposive sampling, } 16 \text { focus group } \\
\text { discussions groups: (fathers of children }<2 \\
\text { years; } 9 \text { mothers of children }<2 \text { years; } \\
\text { grandmothers of children }<2 \text { years; } \\
\text { mothers of children }<2 \text { years; and on } \\
\text { maternal nutrition for mothers of children } \\
<2 \text { years) }\end{array}$ & $\begin{array}{l}\text { 6. no discussion about the researcher's } \\
\text { role, potential bias and influence }\end{array}$ \\
\hline
\end{tabular}


Table 1. Cont.

\begin{tabular}{|c|c|c|c|c|c|c|c|}
\hline No. & $\begin{array}{l}\text { Author, Year, and } \\
\text { Country of Study }\end{array}$ & Title & Source Type & Aims of the Study & $\begin{array}{l}\text { Study Design, Analysis Method } \\
\text { (AM) }\end{array}$ & Sampling Method, Sample Size & $\begin{array}{c}\text { Assessment of Methodological } \\
\text { Limitations of Study (Numbers Refer } \\
\text { to CASP Questions) }\end{array}$ \\
\hline 22 & $\begin{array}{l}\text { United States Agency } \\
\text { for International } \\
\text { Development } \\
2011 \\
\text { Ghana }\end{array}$ & $\begin{array}{l}\text { Ghana promotion of } \\
\text { complementary feeding } \\
\text { practices project-baseline } \\
\text { survey report }\end{array}$ & $\begin{array}{l}\text { United States } \\
\text { Agency for } \\
\text { International } \\
\text { Development }\end{array}$ & $\begin{array}{l}\text { To provide an in-depth analysis of } \\
\text { infant and young child feeding } \\
\text { knowledge, practices, and behaviors } \\
\text { as a abeline to guide the } \\
\text { implementation of the Ghana } \\
\text { Promotion of Complementary } \\
\text { Feeding Practices Project, and to } \\
\text { provide a basis for monitoring and } \\
\text { evaluating the effectiveness of the } \\
\text { behavior change } \\
\text { communication campaign }\end{array}$ & $\begin{array}{l}\text { Mixed method study (in-depth } \\
\text { interviews and focus } \\
\text { group discussion) } \\
\text { AM: Thematic analysis; } \\
\text { triangulation of different } \\
\text { data sources }\end{array}$ & $\begin{array}{l}\text { Purposive and convenience selection, } 20 \\
\text { focus group discussions with fathers of } \\
\text { children 6-24 months and } 30 \text { focus group } \\
\text { discussions with mothers/caregivers of } \\
\text { children 6-24 months. }\end{array}$ & $\begin{array}{l}\text { 5. no explanation regarding the form } \\
\text { of data) } \\
\text { 6. no discussion about the researcher's } \\
\text { role, potential bias and influence } \\
\text { 7. no information about approval from } \\
\text { the ethics committee }\end{array}$ \\
\hline 23 & $\begin{array}{l}\text { Alive and Thrive } \\
\text { Bangladesh } \\
2012 \\
\text { Bangladesh }\end{array}$ & $\begin{array}{l}\text { Perceptions, practices, and } \\
\text { promotion of infant and } \\
\text { young child feeding-results } \\
\text { and program implications of } \\
\text { assessments in Bangladesh }\end{array}$ & $\begin{array}{l}\text { Alive and } \\
\text { Thrive } \\
\text { Bangladesh }\end{array}$ & $\begin{array}{l}\text { "To determine the perceptions of } \\
\text { mothers, influential family and } \\
\text { community members and } \\
\text { understanding of community-level } \\
\text { health care providers of IYCF." to } \\
\text { assess constraints and motivators as } \\
\text { well as sources of information } \\
\text { on IYCF }\end{array}$ & $\begin{array}{l}\text { Mixed method Study (in-depth } \\
\text { interviews and focus } \\
\text { group discussion) } \\
\text { AM: Not stated }\end{array}$ & $\begin{array}{l}\text { Purposive convenience sampling } \\
\text { Semi-structured interviews with } 42 \\
\text { mothers of children } 0-23.9 \text { months and } 28 \\
\text { focus group discussions with fathers and } \\
\text { grandmothers }\end{array}$ & $\begin{array}{l}\text { 5. no explanation regarding the form } \\
\text { of data } \\
\text { 6. no discussion about the researcher's } \\
\text { role, potential bias and influence } \\
\text { 7. no information about approval from } \\
\text { the ethics committee } \\
\text { 8. no information about the process } \\
\text { of analysis }\end{array}$ \\
\hline
\end{tabular}




\subsection{Quality Appraisal}

Each selected document was initially assessed for quality and internal validity according to the Critical Appraisal Skills Program (CASP) checklist for qualitative research [22]. The CASP checklist includes 10 questions to appraise the quality of qualitative research. These assessments for each study can be seen in the final column of Table 1 with reference to the CASP appraisal question number where the study presented potential quality limitations. Selected studies met minimum criteria defined through the checklist including domains such as appropriateness of study design, data collection techniques, and analysis methods used. CASP questions include the following:

1. Was there a clear statement of the aims of the research?

2. Is a qualitative methodology appropriate?

3. Was the research design appropriate to address the aims of the research?

4. Was the recruitment strategy appropriate to the aims of the research?

5. Was the data collected in a way that addressed the research issue?

6. Has the relationship between researcher and participants been adequately considered?

7. Have critical issues been taken into consideration?

8. Was the data analysis sufficiently rigorous?

9. Is there a clear statement of findings?

10. How valuable is the research?

At the second level of appraisal, the GRADE-CERQual (Grade Conidence Evidencerom Reviews Qualitative research) guidance [23] was used to differentiate emergent findings strongly supported or less well supported. Two reviewers independently reviewed studies. Reviewers used guidance derived from GRADE-CERQual to reach consensus of the quality of findings emergent from included studies. The results of this appraisal are presented in Table 2. During this process, methodological limitations of emergent findings were considered and categorized as minor, moderate or major. These categorizations were based on the presence or absence of description of key elements in methodology (guided by the CASP assessment in Table 1) in the document(s) which support the finding, including the following: approach used to recruit or sample participants, how potential for researcher bias was addressed, how analysis was done or, in the case of mixed methods research, how statistical sampling and analysis was done. Minor methodological limitations were those with one element missing, moderate limitations were those with two elements missing, and major limitations were those with three or more missing. Full details of which elements were missing from each document are available in Appendix C. Additional assessments of emergent findings were conducted based on the quality of the studies or documents supporting the finding. These included assessments of relevance, coherence and adequacy guided by the GRADE CERQual methodology. Assessments of relevance and coherence were not conducted for emergent findings with minimal (usually one) studies or grey literature contributing to the finding. These assessments for each emergent finding are given in Table 2 with full details of the how assessments were made found in Appendix $C$. The overall assessment of confidence in emergent findings was based upon the assessments of methodological limitations, relevance, coherence and adequacy and was guided by the GRADE CERQual method. These are given for each emergent finding in Table 2 along with an explanation of judgment describing how the level of confidence was reached. 
Table 2. CERQual qualitative evidence profile of identified barriers and facilitators.

\begin{tabular}{|c|c|c|c|c|c|c|c|c|}
\hline $\begin{array}{l}\text { Finding } \\
\text { No. }\end{array}$ & Review Finding & $\begin{array}{l}\text { Studies } \\
\text { Contributing to the } \\
\text { Review Finding } \\
\end{array}$ & $\begin{array}{l}\text { Assessment of } \\
\text { Methodological } \\
\text { Limitations }\end{array}$ & $\begin{array}{c}\text { Assessment of } \\
\text { Relevance }\end{array}$ & $\begin{array}{l}\text { Assessment of } \\
\text { Coherence }\end{array}$ & $\begin{array}{l}\text { Assessment of } \\
\text { Adequacy }\end{array}$ & $\begin{array}{l}\text { Overall CERQual } \\
\text { Assessment of } \\
\text { Confidence } \\
\end{array}$ & Explanation of Judgement \\
\hline \multicolumn{9}{|c|}{ Barriers } \\
\hline \multicolumn{9}{|c|}{$\begin{array}{l}\text { Barriers to Recommended Breastfeeding } \\
\text { Key Theme: Beliefs and Perceptions }\end{array}$} \\
\hline 1 & $\begin{array}{l}\text { Breastfeeding alters a } \\
\text { woman's breasts in an } \\
\text { undesirable way }\end{array}$ & $1 ; 4 ; 13$ & $\begin{array}{l}\text { Minor } \\
\text { methodological } \\
\text { limitations }\end{array}$ & $\begin{array}{r}\text { Key Theme } \\
\begin{array}{l}\text { Minor concerns } \\
\text { about relevance }\end{array}\end{array}$ & $\begin{array}{l}\text { Beliefs and Perceptio } \\
\text { Minor concerns } \\
\text { about coherence }\end{array}$ & $\begin{array}{l}\text { No concern about } \\
\text { adequacy of data }\end{array}$ & High confidence & $\begin{array}{l}\text { This finding was graded as high confidence because of } \\
\text { few concerns about coherence and relevance and } \\
\text { moderate methodological limitations. }\end{array}$ \\
\hline 2 & $\begin{array}{l}\text { Breastfeeding for more than } \\
\text { one year makes children } \\
\text { susceptible to infection }\end{array}$ & 11 & $\begin{array}{l}\text { Minor } \\
\text { methodological } \\
\text { limitations }\end{array}$ & NA & NA & $\begin{array}{l}\text { Minor concern } \\
\text { about adequacy }\end{array}$ & NA & NA \\
\hline 3 & $\begin{array}{l}\text { Breastmilk is unhealthy for } \\
\text { baby if produced while a } \\
\text { mother is angry }\end{array}$ & 11 & $\begin{array}{l}\text { Minor } \\
\text { methodological } \\
\text { limitations }\end{array}$ & NA & NA & $\begin{array}{l}\text { Minor concern } \\
\text { about adequacy }\end{array}$ & NA & NA \\
\hline 4 & $\begin{array}{l}\text { Breastmilk is unhealthy for } \\
\text { baby if produced while a } \\
\text { mother has an illness }\end{array}$ & 11 & $\begin{array}{l}\text { Minor } \\
\text { methodological } \\
\text { limitations }\end{array}$ & NA & NA & $\begin{array}{l}\text { Minor concern } \\
\text { about adequacy }\end{array}$ & NA & NA \\
\hline 5 & $\begin{array}{l}\text { Breastmilk is not enough for } \\
\text { a small baby }\end{array}$ & $8 ; 16$ & $\begin{array}{l}\text { Substantial } \\
\text { methodological } \\
\text { limitations }\end{array}$ & $\begin{array}{l}\text { Moderate } \\
\text { concerns about } \\
\text { relevance }\end{array}$ & $\begin{array}{l}\text { Minor concerns } \\
\text { about coherence }\end{array}$ & $\begin{array}{l}\text { Moderate } \\
\text { concerns about } \\
\text { adequacy }\end{array}$ & Low confidence & $\begin{array}{l}\text { This finding was graded as low confidence because of } \\
\text { moderate concerns about relevance and adequacy of data } \\
\text { and substantial methodological limitations. }\end{array}$ \\
\hline 6 & $\begin{array}{l}\text { When baby belches on the } \\
\text { breast during breastfeeding } \\
\text { the mother can develop } \\
\text { swollen breasts }\end{array}$ & 4 & $\begin{array}{l}\text { Moderate } \\
\text { methodological } \\
\text { limitations }\end{array}$ & NA & NA & $\begin{array}{l}\text { No concern about } \\
\text { adequacy of data }\end{array}$ & NA & NA \\
\hline 7 & $\begin{array}{l}\text { Colostrum is not healthy for } \\
\text { baby, so mothers discard } \\
\text { colostrum }\end{array}$ & $3 ; 7 ; 10 ; 18$ & $\begin{array}{l}\text { Moderate } \\
\text { methodological } \\
\text { limitations }\end{array}$ & $\begin{array}{l}\text { Minor concerns } \\
\text { about relevance }\end{array}$ & $\begin{array}{l}\text { Minor concerns } \\
\text { about coherence }\end{array}$ & $\begin{array}{l}\text { Minor concerns } \\
\text { about adequacy }\end{array}$ & $\begin{array}{l}\text { Moderate } \\
\text { confidence }\end{array}$ & $\begin{array}{l}\text { This finding was graded as moderate confidence because } \\
\text { of minor concerns regarding relevance, coherence, and } \\
\text { adequacy and moderate methodological limitations. }\end{array}$ \\
\hline 8 & $\begin{array}{l}\text { Baby remains hungry after } \\
\text { breastfeeding (e.g., a baby is } \\
\text { fussy even after } \\
\text { breastfeeding) }\end{array}$ & $\begin{array}{c}5 ; 6 ; 8 ; 9 ; 14 ; 15 ; 16 ; 17 \\
19 ; 21\end{array}$ & $\begin{array}{l}\text { Moderate } \\
\text { methodological } \\
\text { limitations }\end{array}$ & $\begin{array}{l}\text { Minor concerns } \\
\text { about relevance }\end{array}$ & $\begin{array}{l}\text { Minor concerns } \\
\text { about coherence }\end{array}$ & $\begin{array}{l}\text { Moderate } \\
\text { concerns about } \\
\text { adequacy }\end{array}$ & $\begin{array}{l}\text { Moderate } \\
\text { confidence }\end{array}$ & $\begin{array}{l}\text { This finding was graded as moderate confidence because } \\
\text { of moderate concerns regarding methodological } \\
\text { limitations and adequacy of data and minor concerns } \\
\text { about relevance and coherence. }\end{array}$ \\
\hline 9 & $\begin{array}{l}\text { Breast milk substitutes can } \\
\text { make a baby healthier }\end{array}$ & 8 & $\begin{array}{l}\text { Moderate } \\
\text { methodological } \\
\text { limitations }\end{array}$ & NA & NA & $\begin{array}{l}\text { No concern about } \\
\text { adequacy }\end{array}$ & NA & NA \\
\hline
\end{tabular}


Table 2. Cont.

\begin{tabular}{|c|c|c|c|c|c|c|c|c|}
\hline $\begin{array}{l}\text { Finding } \\
\text { No. }\end{array}$ & Review Finding & $\begin{array}{c}\text { Studies } \\
\text { Contributing to the } \\
\text { Review Finding }\end{array}$ & $\begin{array}{l}\text { Assessment of } \\
\text { Methodological } \\
\text { Limitations }\end{array}$ & $\begin{array}{l}\text { Assessment of } \\
\text { Relevance }\end{array}$ & $\begin{array}{l}\text { Assessment of } \\
\text { Coherence }\end{array}$ & $\begin{array}{l}\text { Assessment of } \\
\text { Adequacy }\end{array}$ & $\begin{array}{l}\text { Overall CERQual } \\
\text { Assessment of } \\
\text { Confidence }\end{array}$ & Explanation of Judgement \\
\hline 10 & $\begin{array}{l}\text { Mothers do not produce } \\
\text { sufficient breastmilk }\end{array}$ & $\begin{array}{l}3 ; 4 ; 5 ; 6 ; 7 ; 8 ; 9 ; 13 ; 14 ; \\
\quad 16 ; 17 ; 19 ; 21 ; 23\end{array}$ & $\begin{array}{l}\text { Moderate } \\
\text { methodological } \\
\text { limitations }\end{array}$ & $\begin{array}{l}\text { Minor concerns } \\
\text { about relevance }\end{array}$ & $\begin{array}{l}\text { Minor concerns } \\
\text { about coherence }\end{array}$ & $\begin{array}{l}\text { Minor concerns } \\
\text { about adequacy }\end{array}$ & High confidence & $\begin{array}{l}\text { This finding was graded as high confidence because of } \\
\text { minor concerns about relevance, coherence and adequacy } \\
\text { and moderate methodological limitations. }\end{array}$ \\
\hline 11 & $\begin{array}{l}\text { Babies are thirsty and } \\
\text { need water }\end{array}$ & $\begin{array}{c}2 ; 7 ; 9 ; 13 ; 14 ; 16 ; 17 ; \\
19 ; 21 ; 23\end{array}$ & $\begin{array}{l}\text { Moderate } \\
\text { methodological } \\
\text { limitations }\end{array}$ & $\begin{array}{l}\text { Minor concerns } \\
\text { about relevance }\end{array}$ & $\begin{array}{l}\text { Minor concerns } \\
\text { about coherence }\end{array}$ & $\begin{array}{l}\text { Moderate } \\
\text { concerns about } \\
\text { adequacy }\end{array}$ & $\begin{array}{l}\text { Moderate } \\
\text { confidence }\end{array}$ & $\begin{array}{l}\text { This finding was graded as moderate confidence because } \\
\text { of moderate concerns regarding methodological } \\
\text { limitations and adequacy of data and minor concerns } \\
\text { about relevance and coherence. }\end{array}$ \\
\hline 12 & $\begin{array}{l}\text { Other liquids given shortly } \\
\text { after birth to clean the infant, } \\
\text { believing the insides need to } \\
\text { be purged }\end{array}$ & 11 & $\begin{array}{l}\text { Minor } \\
\text { methodological } \\
\text { limitations }\end{array}$ & NA & $\mathrm{NA}$ & $\begin{array}{l}\text { Substantial } \\
\text { concerns about } \\
\text { adequacy }\end{array}$ & NA & $\mathrm{NA}$ \\
\hline 13 & $\begin{array}{l}\text { Delay in breastmilk "coming } \\
\text { in" following the birth } \\
\text { (delayed onset of } \\
\text { Lactogenesis II) }\end{array}$ & 3 & $\begin{array}{l}\text { Moderate } \\
\text { methodological } \\
\text { limitations }\end{array}$ & $\mathrm{NA}$ & NA & $\begin{array}{l}\text { No concern about } \\
\text { adequacy of data }\end{array}$ & $\mathrm{NA}$ & NA \\
\hline 14 & $\begin{array}{l}\text { Giving traditional herbs to } \\
\text { newborns or young babies is } \\
\text { a deeply rooted practice }\end{array}$ & 20 & $\begin{array}{l}\text { Moderate } \\
\text { methodological } \\
\text { limitations }\end{array}$ & NA & NA & $\begin{array}{l}\text { Minor concerns } \\
\text { about adequacy }\end{array}$ & NA & $\mathrm{NA}$ \\
\hline 15 & $\begin{array}{l}\text { Mothers do not have } \\
\text { confidence that they are } \\
\text { producing sufficient breast } \\
\text { milk to meet baby's needs }\end{array}$ & 5 & $\begin{array}{l}\text { Moderate } \\
\text { methodological } \\
\text { limitations }\end{array}$ & NA & NA & $\begin{array}{l}\text { No concern about } \\
\text { adequacy of data }\end{array}$ & NA & NA \\
\hline 16 & $\begin{array}{l}\text { Baby still cries even after } \\
\text { mother breastfeeds }\end{array}$ & 21 & $\begin{array}{l}\text { Moderate } \\
\text { methodological } \\
\text { limitations }\end{array}$ & NA & NA & $\begin{array}{l}\text { No concern about } \\
\text { adequacy of data }\end{array}$ & NA & NA \\
\hline 17 & $\begin{array}{l}\text { Herbal infusions in water } \\
\text { prevent a baby from } \\
\text { getting ill }\end{array}$ & 18 & $\begin{array}{l}\text { Moderate } \\
\text { methodological } \\
\text { limitations }\end{array}$ & NA & NA & $\begin{array}{l}\text { No concern about } \\
\text { adequacy of data }\end{array}$ & NA & $\mathrm{NA}$ \\
\hline 18 & $\begin{array}{l}\text { Exclusive breastfeeding is } \\
\text { not part of local tradition }\end{array}$ & $\begin{array}{c}2 ; 7 ; 14 ; 16 ; 17 ; 18 ; 19 ; \\
21 ; 22\end{array}$ & $\begin{array}{l}\text { Moderate } \\
\text { methodological } \\
\text { limitations }\end{array}$ & $\begin{array}{l}\text { Minor concerns } \\
\text { about relevance }\end{array}$ & $\begin{array}{l}\text { Moderate } \\
\text { concerns about } \\
\text { coherence }\end{array}$ & $\begin{array}{l}\text { Moderate } \\
\text { concerns about } \\
\text { adequacy of data }\end{array}$ & $\begin{array}{l}\text { Moderate } \\
\text { confidence }\end{array}$ & $\begin{array}{l}\text { This finding was graded as moderate confidence because } \\
\text { of minor concerns about relevance and moderate } \\
\text { concerns regarding methodological limitations and } \\
\text { coherence and adequacy of data. }\end{array}$ \\
\hline 19 & $\begin{array}{l}\text { Mothers attribute their } \\
\text { inability to produce enough } \\
\text { breastmilk to their own diet }\end{array}$ & $\begin{array}{l}11 ; 13 ; 14 ; 17 ; 18 ; 21 ; \\
23\end{array}$ & $\begin{array}{l}\text { Moderate } \\
\text { methodological } \\
\text { limitations }\end{array}$ & $\begin{array}{l}\text { Minor concerns } \\
\text { about relevance }\end{array}$ & $\begin{array}{l}\text { Minor concerns } \\
\text { about coherence }\end{array}$ & $\begin{array}{l}\text { Minor concerns } \\
\text { about adequacy of } \\
\text { data }\end{array}$ & High confidence & $\begin{array}{l}\text { This finding was graded as high confidence because of } \\
\text { minor concerns about relevance, coherence and adequacy } \\
\text { and moderate methodological limitations. }\end{array}$ \\
\hline 20 & $\begin{array}{l}\text { Women should cease } \\
\text { breastfeeding if they become } \\
\text { pregnant before nursling is } 2 \\
\text { years old }\end{array}$ & $15 ; 17 ; 18 ; 21$ & $\begin{array}{l}\text { Moderate } \\
\text { methodological } \\
\text { limitations }\end{array}$ & $\begin{array}{l}\text { Minor concerns } \\
\text { about relevance }\end{array}$ & $\begin{array}{l}\text { Minor concerns } \\
\text { about coherence }\end{array}$ & $\begin{array}{l}\text { Moderate } \\
\text { concerns about } \\
\text { adequacy of data }\end{array}$ & $\begin{array}{l}\text { Moderate } \\
\text { confidence }\end{array}$ & $\begin{array}{l}\text { This finding was graded as moderate confidence because } \\
\text { of minor concerns about relevance, coherence and } \\
\text { moderate concerns about methodological limitations and } \\
\text { adequacy of data. }\end{array}$ \\
\hline
\end{tabular}


Table 2. Cont

\begin{tabular}{|c|c|c|c|c|c|c|c|c|}
\hline $\begin{array}{l}\text { Finding } \\
\text { No. }\end{array}$ & Review Finding & $\begin{array}{c}\text { Studies } \\
\text { Contributing to the } \\
\text { Review Finding }\end{array}$ & $\begin{array}{c}\text { Assessment of } \\
\text { Methodological } \\
\text { Limitations } \\
\end{array}$ & $\begin{array}{c}\text { Assessment of } \\
\text { Relevance }\end{array}$ & $\begin{array}{l}\text { Assessment of } \\
\text { Coherence }\end{array}$ & $\begin{array}{l}\text { Assessment of } \\
\text { Adequacy }\end{array}$ & $\begin{array}{l}\text { Overall CERQual } \\
\text { Assessment of } \\
\text { Confidence }\end{array}$ & Explanation of Judgement \\
\hline \multicolumn{9}{|c|}{ Key Theme: Lack of Support from Families, Health Workers (HWs) and others } \\
\hline 21 & $\begin{array}{l}\text { Family members do not } \\
\text { support ideal } \\
\text { breastfeeding practices }\end{array}$ & $4 ; 19 ; 20 ; 21$ & $\begin{array}{l}\text { Moderate } \\
\text { methodological } \\
\text { limitations }\end{array}$ & $\begin{array}{l}\text { Minor concerns } \\
\text { about relevance }\end{array}$ & $\begin{array}{l}\text { Minor concerns } \\
\text { about coherence }\end{array}$ & $\begin{array}{l}\text { Substantial } \\
\text { concerns about } \\
\text { adequacy }\end{array}$ & $\begin{array}{l}\text { Moderate } \\
\text { confidence }\end{array}$ & $\begin{array}{l}\text { This finding was graded as moderate confidence because } \\
\text { of minor concerns about coherence and relevance; } \\
\text { moderate methodological limitations; and substantial } \\
\text { concerns about adequacy. }\end{array}$ \\
\hline 22 & $\begin{array}{l}\text { Mothers do not have enough } \\
\text { time to breastfeed their } \\
\text { infants due to work }\end{array}$ & $\begin{array}{c}4 ; 6 ; 7 ; 8 ; 9 ; 13 ; 14 ; 15 ; \\
16 ; 1 ; ; 18 ; 20\end{array}$ & $\begin{array}{l}\text { Moderate } \\
\text { methodological } \\
\text { limitations }\end{array}$ & $\begin{array}{l}\text { Minor concerns } \\
\text { about relevance }\end{array}$ & $\begin{array}{l}\text { Minor concerns } \\
\text { about } \\
\text { coherence) }\end{array}$ & $\begin{array}{l}\text { Moderate } \\
\text { concerns about } \\
\text { adequacy }\end{array}$ & $\begin{array}{l}\text { Moderate } \\
\text { confidence }\end{array}$ & $\begin{array}{l}\text { This finding was graded as moderate confidence because } \\
\text { of minor concerns about relevance and moderate } \\
\text { concerns regarding methodological limitations and } \\
\text { coherence and adequacy of data. }\end{array}$ \\
\hline 23 & $\begin{array}{l}\text { HWs do not reassure women } \\
\text { about their ability } \\
\text { to breastfeed }\end{array}$ & 3 & $\begin{array}{l}\text { Moderate } \\
\text { methodological } \\
\text { limitations }\end{array}$ & NA & NA & $\begin{array}{l}\text { No concern about } \\
\text { adequacy of data }\end{array}$ & NA & NA \\
\hline 24 & $\begin{array}{l}\text { HWs do not have adequate } \\
\text { knowledge and skills to } \\
\text { support mothers }\end{array}$ & $2 ; 9 ; 23$ & $\begin{array}{l}\text { Moderate } \\
\text { methodological } \\
\text { limitations }\end{array}$ & $\begin{array}{l}\text { Moderate } \\
\text { concerns about } \\
\text { relevance }\end{array}$ & $\begin{array}{l}\text { Minor concerns } \\
\text { about coherence }\end{array}$ & $\begin{array}{l}\text { Minor concerns } \\
\text { about adequacy }\end{array}$ & $\begin{array}{l}\text { Moderate } \\
\text { confidence }\end{array}$ & $\begin{array}{l}\text { This finding was graded as moderate confidence because } \\
\text { of minor concerns about coherence and adequacy; } \\
\text { moderate concern about relevance and methodological } \\
\text { limitations. }\end{array}$ \\
\hline 25 & $\begin{array}{l}\text { Mothers do not receive } \\
\text { advice from HWs }\end{array}$ & $3 ; 9 ; 10 ; 13$ & $\begin{array}{l}\text { Minor } \\
\text { methodological } \\
\text { limitations }\end{array}$ & $\begin{array}{l}\text { Minor concerns } \\
\text { about relevance }\end{array}$ & $\begin{array}{l}\text { Minor concerns } \\
\text { about coherence }\end{array}$ & $\begin{array}{l}\text { Minor concerns } \\
\text { about adequacy }\end{array}$ & High confidence & $\begin{array}{l}\text { This finding was graded as high confidence because of } \\
\text { few concerns about methodological limitations, } \\
\text { relevance, coherence and adequacy. }\end{array}$ \\
\hline 26 & $\begin{array}{l}\text { Media has a negative impact } \\
\text { on breastfeeding (promoting } \\
\text { breastmilk substitutes as } \\
\text { healthy for babies) }\end{array}$ & $7 ; 8 ; 13$ & $\begin{array}{l}\text { Moderate } \\
\text { methodological } \\
\text { limitations }\end{array}$ & $\begin{array}{l}\text { Minor concerns } \\
\text { about relevance }\end{array}$ & $\begin{array}{l}\text { Minor concerns } \\
\text { about coherence }\end{array}$ & $\begin{array}{l}\text { No concern about } \\
\text { adequacy of data }\end{array}$ & High confidence & $\begin{array}{l}\text { This finding was graded as high confidence because of } \\
\text { few concerns about coherence and relevance and } \\
\text { moderate methodological limitations. }\end{array}$ \\
\hline 27 & $\begin{array}{l}\text { Traditional beliefs and } \\
\text { influence of older female } \\
\text { family members (e.g., } \\
\text { grandmothers) } \\
\text { influence breastfeeding }\end{array}$ & $7 ; 16 ; 18 ; 21$ & $\begin{array}{l}\text { Moderate } \\
\text { methodological } \\
\text { limitations }\end{array}$ & $\begin{array}{l}\text { Minor concerns } \\
\text { about relevance }\end{array}$ & $\begin{array}{l}\text { Minor concerns } \\
\text { about coherence }\end{array}$ & $\begin{array}{l}\text { Moderate } \\
\text { concerns about } \\
\text { adequacy }\end{array}$ & $\begin{array}{l}\text { Moderate } \\
\text { confidence }\end{array}$ & $\begin{array}{l}\text { This finding was graded as moderate confidence because } \\
\text { of minor concerns about coherence and relevance; } \\
\text { moderate concern about methodological limitations } \\
\text { and adequacy. }\end{array}$ \\
\hline 28 & $\begin{array}{l}\text { Comments/advice from } \\
\text { families, peers or close } \\
\text { neighbors } \\
\text { influence breastfeeding }\end{array}$ & $8 ; 9 ; 13$ & $\begin{array}{l}\text { Moderate } \\
\text { methodological } \\
\text { limitations }\end{array}$ & $\begin{array}{l}\text { Minor concerns } \\
\text { about relevance }\end{array}$ & $\begin{array}{l}\text { Minor concerns } \\
\text { about coherence }\end{array}$ & $\begin{array}{l}\text { No concern about } \\
\text { adequacy of data }\end{array}$ & High confidence & $\begin{array}{l}\text { This finding was graded as high confidence because of } \\
\text { adequate data, minor concerns about coherence and } \\
\text { relevance and moderate concerns about } \\
\text { methodological limitations. }\end{array}$ \\
\hline \multicolumn{9}{|c|}{ Key Theme: Infant-Specific Factors } \\
\hline 29 & $\begin{array}{l}\text { Babies stop breastfeeding for } \\
\text { unknown reasons (lack of } \\
\text { interest in feeding, } \\
\text { self-weaning) }\end{array}$ & $9 ; 13$ & $\begin{array}{l}\text { Moderate } \\
\text { methodological } \\
\text { limitations }\end{array}$ & $\begin{array}{l}\text { Moderate } \\
\text { concerns about } \\
\text { relevance }\end{array}$ & $\begin{array}{l}\text { Minor concerns } \\
\text { about coherence }\end{array}$ & $\begin{array}{l}\text { No concern about } \\
\text { adequacy of data }\end{array}$ & $\begin{array}{l}\text { Moderate } \\
\text { confidence }\end{array}$ & $\begin{array}{l}\text { This finding was graded as moderate confidence because } \\
\text { of minor concerns about coherence, moderate concern } \\
\text { about relevance and methodological limitations. }\end{array}$ \\
\hline 30 & $\begin{array}{l}\text { Delayed breastfeeding } \\
\text { initiation of infants with } \\
\text { respiratory problems }\end{array}$ & 10 & $\begin{array}{l}\text { Minor } \\
\text { methodological } \\
\text { limitations }\end{array}$ & NA & NA & $\begin{array}{l}\text { No concern about } \\
\text { adequacy of data }\end{array}$ & NA & NA \\
\hline 31 & Baby has an illness & $4 ; 10 ; 14 ; 17 ; 19$ & $\begin{array}{l}\text { Moderate } \\
\text { methodological } \\
\text { limitations. }\end{array}$ & $\begin{array}{l}\text { Minor concerns } \\
\text { about relevance }\end{array}$ & $\begin{array}{l}\text { Minor concerns } \\
\text { about coherence }\end{array}$ & $\begin{array}{l}\text { Substantial } \\
\text { concerns about } \\
\text { adequacy }\end{array}$ & $\begin{array}{l}\text { Moderate } \\
\text { confidence }\end{array}$ & $\begin{array}{l}\text { This finding was graded as moderate confidence because } \\
\text { of substantial concerns about adequacy; moderate } \\
\text { methodological limitations; and minor concerns } \\
\text { regarding coherence and relevance. }\end{array}$ \\
\hline
\end{tabular}


Table 2. Cont

\begin{tabular}{|c|c|c|c|c|c|c|c|c|}
\hline $\begin{array}{l}\text { Finding } \\
\text { No. }\end{array}$ & Review Finding & $\begin{array}{c}\text { Studies } \\
\text { Contributing to the } \\
\text { Review Finding } \\
\end{array}$ & $\begin{array}{c}\text { Assessment of } \\
\text { Methodological } \\
\text { Limitations } \\
\end{array}$ & $\begin{array}{l}\text { Assessment of } \\
\text { Relevance }\end{array}$ & $\begin{array}{l}\text { Assessment of } \\
\text { Coherence }\end{array}$ & $\begin{array}{l}\text { Assessment of } \\
\text { Adequacy }\end{array}$ & $\begin{array}{l}\text { Overall CERQual } \\
\text { Assessment of } \\
\text { Confidence }\end{array}$ & Explanation of Judgement \\
\hline \multicolumn{9}{|c|}{ Key Theme: Mothers' Understanding } \\
\hline 32 & $\begin{array}{l}\text { Mothers do not understand } \\
\text { purpose of colostrum and } \\
\text { breastfeeding initiation }\end{array}$ & $3 ; 7 ; 8 ; 14 ; 17 ; 18 ; 20$ & $\begin{array}{l}\text { Moderate } \\
\text { methodological } \\
\text { limitations }\end{array}$ & $\begin{array}{l}\text { Minor concerns } \\
\text { about relevance }\end{array}$ & $\begin{array}{l}\text { Minor concerns } \\
\text { about coherence }\end{array}$ & $\begin{array}{l}\text { Substantial } \\
\text { concerns about } \\
\text { adequacy }\end{array}$ & $\begin{array}{l}\text { Moderate } \\
\text { confidence }\end{array}$ & $\begin{array}{l}\text { This finding was graded as moderate confidence because } \\
\text { of substantial concerns about adequacy; moderate } \\
\text { methodological limitations; and minor concerns } \\
\text { regarding coherence and relevance. }\end{array}$ \\
\hline 33 & $\begin{array}{l}\text { Mothers do not have } \\
\text { technical knowledge about } \\
\text { breastfeeding practices }\end{array}$ & $\begin{array}{l}8 ; 9 ; 10 ; 13 ; 14 ; 15 ; 17 \\
\quad 18 ; 20 ; 21 ; 22\end{array}$ & $\begin{array}{l}\text { Moderate } \\
\text { methodological } \\
\text { limitations }\end{array}$ & $\begin{array}{l}\text { Minor concerns } \\
\text { about relevance }\end{array}$ & $\begin{array}{l}\text { Moderate } \\
\text { concerns about } \\
\text { coherence }\end{array}$ & $\begin{array}{l}\text { Moderate } \\
\text { concerns about } \\
\text { adequacy }\end{array}$ & $\begin{array}{l}\text { Moderate } \\
\text { confidence }\end{array}$ & $\begin{array}{l}\text { This finding was graded as moderate confidence because } \\
\text { of moderate concerns regarding methodology, coherence, } \\
\text { adequacy and minor concerns about relevance. }\end{array}$ \\
\hline 34 & $\begin{array}{l}\text { Mothers unable to interpret } \\
\text { child's feeding cues } \\
\text { or behavior }\end{array}$ & $8 ; 17 ; 19$ & $\begin{array}{l}\text { Moderate } \\
\text { methodological } \\
\text { limitations }\end{array}$ & $\begin{array}{l}\text { Moderate } \\
\text { concerns about } \\
\text { relevance }\end{array}$ & $\begin{array}{l}\text { Minor concerns } \\
\text { about coherence }\end{array}$ & $\begin{array}{l}\text { Minor concerns } \\
\text { about adequacy }\end{array}$ & $\begin{array}{l}\text { Moderate } \\
\text { confidence }\end{array}$ & $\begin{array}{l}\text { This finding was graded as moderate confidence because } \\
\text { of moderate concerns about methodology, relevance, } \\
\text { coherence and minor concerns about adequacy. }\end{array}$ \\
\hline 35 & $\begin{array}{l}\text { Mothers do not have formal } \\
\text { schooling or education }\end{array}$ & 2 & $\begin{array}{l}\text { Moderate } \\
\text { methodological } \\
\text { limitations }\end{array}$ & $\mathrm{NA}$ & $\mathrm{NA}$ & $\begin{array}{l}\text { Minor concern } \\
\text { about adequacy } \\
\text { of data }\end{array}$ & NA & NA \\
\hline \multicolumn{9}{|c|}{ Key Theme: Mother-Specific Factors } \\
\hline 36 & $\begin{array}{l}\text { Mothers have breast or } \\
\text { nipple problems }\end{array}$ & $4 ; 5 ; 7 ; 9 ; 13 ; 14$ & $\begin{array}{l}\text { Moderate } \\
\text { methodological } \\
\text { limitations }\end{array}$ & $\begin{array}{l}\text { Minor concerns } \\
\text { about relevance }\end{array}$ & $\begin{array}{l}\text { Minor concerns } \\
\text { about coherence }\end{array}$ & $\begin{array}{l}\text { Minor concern } \\
\text { about adequacy } \\
\text { of data }\end{array}$ & High confidence & $\begin{array}{l}\text { This finding was graded as high confidence because of } \\
\text { minor concerns regarding relevance, coherence, } \\
\text { adequacy and moderate methodological limitations. }\end{array}$ \\
\hline 37 & $\begin{array}{l}\text { Mothers have an illness } \\
\text { and/or have died }\end{array}$ & $\begin{array}{c}4 ; 9 ; 12 ; 13 ; 14 ; 15 ; 17 \\
18 ; 21\end{array}$ & $\begin{array}{l}\text { Moderate } \\
\text { methodological } \\
\text { limitations }\end{array}$ & $\begin{array}{l}\text { Minor concerns } \\
\text { about relevance }\end{array}$ & $\begin{array}{l}\text { Minor concerns } \\
\text { about coherence }\end{array}$ & $\begin{array}{l}\text { Substantial } \\
\text { concerns about } \\
\text { adequacy }\end{array}$ & $\begin{array}{l}\text { Moderate } \\
\text { confidence }\end{array}$ & $\begin{array}{l}\text { This finding was graded as moderate confidence because } \\
\text { of substantial concerns about adequacy of data, moderate } \\
\text { methodological limitations and minor concerns } \\
\text { regarding relevance and coherence. }\end{array}$ \\
\hline 38 & $\begin{array}{l}\text { Breastfeeding initiation is } \\
\text { delayed due to } \\
\text { pregnancy/labor-induced } \\
\text { health conditions or activities } \\
\text { needing to be performed } \\
\text { after childbirth }\end{array}$ & $3 ; 10 ; 19$ & $\begin{array}{l}\text { Minor } \\
\text { methodological } \\
\text { limitations }\end{array}$ & $\begin{array}{l}\text { Moderate } \\
\text { concerns about } \\
\text { relevance }\end{array}$ & $\begin{array}{l}\text { Minor concerns } \\
\text { about coherence }\end{array}$ & $\begin{array}{l}\text { Substantial } \\
\text { concerns about } \\
\text { adequacy }\end{array}$ & $\begin{array}{l}\text { Moderate } \\
\text { confidence }\end{array}$ & $\begin{array}{l}\text { This finding was graded as moderate confidence because } \\
\text { of substantial concerns about adequacy, moderate } \\
\text { concerns about relevance and minor concerns about } \\
\text { coherence and methodological limitations. }\end{array}$ \\
\hline \multicolumn{9}{|c|}{ Barrie } \\
\hline 39 & Household food insecurity & $\begin{array}{c}11 ; 12 ; 14 ; 15 ; 16 ; 17 \\
20 ; 21 ; 23\end{array}$ & $\begin{array}{l}\text { Moderate } \\
\text { methodological } \\
\text { limitations }\end{array}$ & $\begin{array}{l}\text { Minor concerns } \\
\text { about relevance }\end{array}$ & $\begin{array}{l}\text { Minor concerns } \\
\text { about coherence }\end{array}$ & $\begin{array}{l}\text { Minor concerns } \\
\text { about adequacy }\end{array}$ & High confidence & $\begin{array}{l}\text { This finding was graded as high confidence because of } \\
\text { minor concerns about relevance, coherence and } \\
\text { adequacy; and moderate methodological limitations. }\end{array}$ \\
\hline 40 & $\begin{array}{l}\text { Replacement of higher } \\
\text { quality foods with lower } \\
\text { quality foods for financial } \\
\text { reasons or drought }\end{array}$ & $12 ; 15$ & $\begin{array}{l}\text { Moderate } \\
\text { methodological } \\
\text { limitations }\end{array}$ & $\begin{array}{l}\text { Moderate } \\
\text { concerns about } \\
\text { relevance }\end{array}$ & $\begin{array}{l}\text { Minor concerns } \\
\text { about coherence }\end{array}$ & $\begin{array}{l}\text { No concern about } \\
\text { adequacy of data }\end{array}$ & $\begin{array}{l}\text { Moderate } \\
\text { confidence }\end{array}$ & $\begin{array}{l}\text { This finding was graded as moderate confidence because } \\
\text { of minor concerns about coherence, moderate concern } \\
\text { about relevance and methodological limitations. }\end{array}$ \\
\hline 41 & $\begin{array}{l}\text { Lack of diversity in } \\
\text { available foods }\end{array}$ & $\begin{array}{c}12 ; 14 ; 15 ; 16 ; 17 ; 19 ; \\
20\end{array}$ & $\begin{array}{l}\text { Moderate } \\
\text { methodological } \\
\text { limitations }\end{array}$ & $\begin{array}{l}\text { Minor concerns } \\
\text { about relevance }\end{array}$ & $\begin{array}{l}\text { Minor concerns } \\
\text { about coherence }\end{array}$ & $\begin{array}{l}\text { Minor concerns } \\
\text { about adequacy }\end{array}$ & High confidence & $\begin{array}{l}\text { This finding was graded as high confidence because of } \\
\text { minor concerns about relevance, coherence and } \\
\text { adequacy; and moderate methodological limitations. }\end{array}$ \\
\hline 42 & Lack of water for cooking & $12 ; 15$ & $\begin{array}{l}\text { Moderate } \\
\text { methodological } \\
\text { limitations }\end{array}$ & $\begin{array}{l}\text { Moderate } \\
\text { concerns about } \\
\text { relevance }\end{array}$ & $\begin{array}{l}\text { Minor concerns } \\
\text { about coherence }\end{array}$ & $\begin{array}{l}\text { No concern about } \\
\text { adequacy of data }\end{array}$ & $\begin{array}{l}\text { Moderate } \\
\text { confidence }\end{array}$ & $\begin{array}{l}\text { This finding was graded as moderate confidence because } \\
\text { of minor concerns about coherence, moderate concern } \\
\text { about relevance and methodological limitations. }\end{array}$ \\
\hline
\end{tabular}


Table 2. Cont

\begin{tabular}{|c|c|c|c|c|c|c|c|c|}
\hline $\begin{array}{l}\text { Finding } \\
\text { No. }\end{array}$ & Review Finding & $\begin{array}{l}\text { Studies } \\
\text { Contributing to the } \\
\text { Review Finding }\end{array}$ & $\begin{array}{l}\text { Assessment of } \\
\text { Methodological } \\
\text { Limitations }\end{array}$ & $\begin{array}{l}\text { Assessment of } \\
\text { Relevance }\end{array}$ & $\begin{array}{l}\text { Assessment of } \\
\text { Coherence }\end{array}$ & $\begin{array}{l}\text { Assessment of } \\
\text { Adequacy }\end{array}$ & $\begin{array}{l}\text { Overall CERQual } \\
\text { Assessment of } \\
\text { Confidence }\end{array}$ & Explanation of Judgement \\
\hline 43 & $\begin{array}{l}\text { Some foods are only } \\
\text { available seasonally }\end{array}$ & 12 & $\begin{array}{l}\text { Minor } \\
\text { methodological } \\
\text { limitations }\end{array}$ & $\mathrm{NA}$ & $\mathrm{NA}$ & $\begin{array}{l}\text { Minor concern } \\
\text { about adequacy of } \\
\text { data }\end{array}$ & $\mathrm{NA}$ & NA \\
\hline 44 & $\begin{array}{l}\text { Mothers prepare foods early } \\
\text { in the day and then store for } \\
\text { later feeding }\end{array}$ & 12 & $\begin{array}{l}\text { Minor } \\
\text { methodological } \\
\text { limitations }\end{array}$ & NA & NA & $\begin{array}{l}\text { No concern about } \\
\text { adequacy of data }\end{array}$ & NA & $\mathrm{NA}$ \\
\hline 45 & $\begin{array}{l}\text { Mothers do not have } \\
\text { financial } \\
\text { decision-making power }\end{array}$ & $14 ; 20$ & $\begin{array}{l}\text { Moderate } \\
\text { methodological } \\
\text { limitations }\end{array}$ & $\begin{array}{l}\text { Moderate } \\
\text { concerns about } \\
\text { relevance }\end{array}$ & $\begin{array}{l}\text { Minor concerns } \\
\text { about coherence }\end{array}$ & $\begin{array}{l}\text { No concern about } \\
\text { adequacy of data }\end{array}$ & $\begin{array}{l}\text { Moderate } \\
\text { confidence }\end{array}$ & $\begin{array}{l}\text { This finding was graded as moderate confidence because } \\
\text { of minor concerns about coherence, moderate concern } \\
\text { about relevance and methodological limitations. }\end{array}$ \\
\hline \multicolumn{9}{|c|}{ Key Theme: Infant-Specific Factors } \\
\hline 46 & $\begin{array}{l}\text { Infants dislike animal } \\
\text { source foods }\end{array}$ & $6 ; 23$ & $\begin{array}{l}\text { Moderate } \\
\text { methodological } \\
\text { limitations }\end{array}$ & $\begin{array}{l}\text { Moderate } \\
\text { concerns about } \\
\text { relevance }\end{array}$ & $\begin{array}{l}\text { Minor concerns } \\
\text { about coherence }\end{array}$ & $\begin{array}{l}\text { Substantial } \\
\text { concern about } \\
\text { adequacy }\end{array}$ & Low confidence & $\begin{array}{l}\text { This finding was graded as low confidence because of } \\
\text { moderate concerns about methodology and relevance; } \\
\text { and substantial concerns about adequacy of data. }\end{array}$ \\
\hline 47 & $\begin{array}{l}\text { Infants do not have appetite } \\
\text { to eat animal source foods }\end{array}$ & $6 ; 18$ & $\begin{array}{l}\text { Moderate } \\
\text { methodological } \\
\text { limitations }\end{array}$ & $\begin{array}{l}\text { Moderate } \\
\text { concerns about } \\
\text { relevance }\end{array}$ & $\begin{array}{l}\text { Minor concerns } \\
\text { about coherence }\end{array}$ & $\begin{array}{l}\text { Moderate concern } \\
\text { about adequacy }\end{array}$ & $\begin{array}{l}\text { Moderate } \\
\text { confidence }\end{array}$ & $\begin{array}{l}\text { This finding was graded as moderate confidence because } \\
\text { of moderate concerns regarding methodological } \\
\text { limitations, adequacy of data, relevance and minor } \\
\text { concerns about coherence. }\end{array}$ \\
\hline 48 & $\begin{array}{l}\text { Infants refuse to eat and or } \\
\text { spit out foods }\end{array}$ & $6 ; 15 ; 23$ & $\begin{array}{l}\text { Moderate } \\
\text { methodological } \\
\text { limitations }\end{array}$ & $\begin{array}{l}\text { Moderate } \\
\text { concerns about } \\
\text { relevance }\end{array}$ & $\begin{array}{l}\text { Moderate } \\
\text { concern about } \\
\text { coherence }\end{array}$ & $\begin{array}{l}\text { Substantial } \\
\text { concerns about } \\
\text { adequacy }\end{array}$ & Low confidence & $\begin{array}{l}\text { This finding was graded as low confidence because of } \\
\text { moderate concerns about relevance and coherence and } \\
\text { substantial concerns about adequacy of data } \\
\text { and methodology. }\end{array}$ \\
\hline 49 & $\begin{array}{l}\text { Infants vomit when animal } \\
\text { source foods are offered }\end{array}$ & $6 ; 15$ & $\begin{array}{l}\text { Substantial } \\
\text { methodological } \\
\text { limitations }\end{array}$ & $\begin{array}{l}\text { Moderate } \\
\text { concerns about } \\
\text { relevance }\end{array}$ & $\begin{array}{l}\text { Moderate } \\
\text { concern about } \\
\text { coherence }\end{array}$ & $\begin{array}{l}\text { Substantial } \\
\text { concerns about } \\
\text { adequacy }\end{array}$ & Low confidence & $\begin{array}{l}\text { This finding was graded as low confidence because of } \\
\text { moderate concerns about relevance and coherence and } \\
\text { substantial concerns about adequacy of data } \\
\text { and methodology. }\end{array}$ \\
\hline 50 & Infants have an illness & $15 ; 18$ & $\begin{array}{l}\text { Moderate } \\
\text { methodological } \\
\text { limitations }\end{array}$ & $\begin{array}{l}\text { Moderate } \\
\text { concerns about } \\
\text { relevance }\end{array}$ & $\begin{array}{l}\text { Minor concerns } \\
\text { about coherence }\end{array}$ & $\begin{array}{l}\text { No concern about } \\
\text { adequacy of data }\end{array}$ & $\begin{array}{l}\text { Moderate } \\
\text { confidence }\end{array}$ & $\begin{array}{l}\text { This finding was graded as moderate confidence because } \\
\text { of moderate concerns about methods and methodology } \\
\text { and minor concerns about coherence. }\end{array}$ \\
\hline \multicolumn{9}{|c|}{ Key Theme: Family-Specific Factors } \\
\hline 51 & $\begin{array}{l}\text { Mothers and other family } \\
\text { members do not have } \\
\text { appropriate knowledge } \\
\text { about } \\
\text { complementary feeding }\end{array}$ & $\begin{array}{c}\text {; } 11 ; 14 ; 15 ; 16 ; 19 ; 20 ; \\
21 ; 23\end{array}$ & $\begin{array}{l}\text { Moderate } \\
\text { methodological } \\
\text { limitations }\end{array}$ & $\begin{array}{l}\text { Minor concerns } \\
\text { about relevance }\end{array}$ & $\begin{array}{l}\text { Minor concerns } \\
\text { about coherence }\end{array}$ & $\begin{array}{l}\text { Minor concerns } \\
\text { about adequacy }\end{array}$ & High confidence & $\begin{array}{l}\text { This finding was graded as high confidence because of } \\
\text { minor concerns about relevance, coherence and } \\
\text { adequacy; and moderate methodological limitations. }\end{array}$ \\
\hline 52 & $\begin{array}{l}\text { Mothers concern about } \\
\text { over-feeding their child } \\
\text { (which might cause them to } \\
\text { be hungrier in future) }\end{array}$ & 16 & $\begin{array}{l}\text { Moderate } \\
\text { methodological } \\
\text { limitations }\end{array}$ & $\mathrm{NA}$ & $\mathrm{NA}$ & $\begin{array}{l}\text { No concern about } \\
\text { adequacy of data }\end{array}$ & NA & NA \\
\hline
\end{tabular}


Table 2. Cont

\begin{tabular}{|c|c|c|c|c|c|c|c|c|}
\hline $\begin{array}{l}\text { Finding } \\
\text { No. }\end{array}$ & Review Finding & $\begin{array}{c}\text { Studies } \\
\text { Contributing to the } \\
\text { Review Finding }\end{array}$ & $\begin{array}{c}\text { Assessment of } \\
\text { Methodological } \\
\text { Limitations }\end{array}$ & $\begin{array}{l}\text { Assessment of } \\
\text { Relevance }\end{array}$ & $\begin{array}{l}\text { Assessment of } \\
\text { Coherence }\end{array}$ & $\begin{array}{l}\text { Assessment of } \\
\text { Adequacy }\end{array}$ & $\begin{array}{l}\text { Overall CERQual } \\
\text { Assessment of } \\
\text { Confidence }\end{array}$ & Explanation of Judgement \\
\hline 53 & $\begin{array}{l}\text { Mothers and family members } \\
\text { fear that child may choke on } \\
\text { certain solid foods }\end{array}$ & $15 ; 16 ; 19$ & $\begin{array}{l}\text { Moderate } \\
\text { methodological } \\
\text { limitations }\end{array}$ & $\begin{array}{l}\text { Moderate } \\
\text { concerns about } \\
\text { relevance }\end{array}$ & $\begin{array}{l}\text { Minor concerns } \\
\text { about coherence }\end{array}$ & $\begin{array}{l}\text { Moderate concern } \\
\text { about adequacy } \\
\text { of data }\end{array}$ & $\begin{array}{l}\text { Moderate } \\
\text { confidence }\end{array}$ & $\begin{array}{l}\text { This finding was graded as moderate confidence because } \\
\text { of moderate concerns about methodology, adequacy of } \\
\text { data, relevance and minor concern regarding coherence. }\end{array}$ \\
\hline 54 & $\begin{array}{l}\text { Religious/cultural traditions } \\
\text { around preparation of } \\
\text { non-fasting foods for } \\
\text { children by adults who } \\
\text { are fasting }\end{array}$ & 15 & $\begin{array}{l}\text { Moderate } \\
\text { methodological } \\
\text { limitations }\end{array}$ & NA & NA & $\begin{array}{l}\text { Moderate concern } \\
\text { about adequacy } \\
\text { of data }\end{array}$ & NA & NA \\
\hline 55 & $\begin{array}{l}\text { Thicker weaning porridge } \\
\text { believed to be difficult for } \\
\text { young children to } \\
\text { swallow/digest }\end{array}$ & $15 ; 16 ; 19 ; 20 ; 23$ & $\begin{array}{l}\text { Moderate } \\
\text { methodological } \\
\text { limitations }\end{array}$ & $\begin{array}{l}\text { Moderate } \\
\text { concerns about } \\
\text { relevance }\end{array}$ & $\begin{array}{l}\text { Minor concerns } \\
\text { about coherence }\end{array}$ & $\begin{array}{l}\text { Moderate concern } \\
\text { about adequacy } \\
\text { of data }\end{array}$ & $\begin{array}{l}\text { Moderate } \\
\text { confidence }\end{array}$ & $\begin{array}{l}\text { This finding was graded as moderate confidence because } \\
\text { of moderate concerns about methodology, adequacy of } \\
\text { data, relevance and minor concern regarding coherence. }\end{array}$ \\
\hline 56 & $\begin{array}{l}\text { Lack of time to cook special } \\
\text { foods for the child }\end{array}$ & $15 ; 16$ & $\begin{array}{l}\text { Moderate } \\
\text { methodological } \\
\text { limitations }\end{array}$ & $\begin{array}{l}\text { Moderate } \\
\text { concerns about } \\
\text { relevance }\end{array}$ & $\begin{array}{l}\text { Minor concerns } \\
\text { about coherence }\end{array}$ & $\begin{array}{l}\text { Minor concern } \\
\text { about adequacy } \\
\text { of data }\end{array}$ & $\begin{array}{l}\text { Moderate } \\
\text { confidence }\end{array}$ & $\begin{array}{l}\text { This finding was graded as moderate confidence because } \\
\text { of moderate concerns about methodology and relevance } \\
\text { and minor concerns regarding coherence and adequacy } \\
\text { of data. }\end{array}$ \\
\hline \multicolumn{9}{|c|}{ Cross-Cutting Barriers } \\
\hline 57 & $\begin{array}{l}\text { Inability of health workers to } \\
\text { provide optimal support } \\
\text { to mothers }\end{array}$ & 15 & $\begin{array}{l}\text { Moderate } \\
\text { methodological } \\
\text { limitations }\end{array}$ & NA & NA & $\begin{array}{l}\text { No concerns } \\
\text { about adequacy } \\
\text { of data }\end{array}$ & NA & NA \\
\hline 58 & Conflicting information & 21 & $\begin{array}{l}\text { Moderate } \\
\text { methodological } \\
\text { limitations }\end{array}$ & NA & NA & $\begin{array}{l}\text { Moderate } \\
\text { concerns }\end{array}$ & NA & NA \\
\hline \multirow{2}{*}{\multicolumn{9}{|c|}{$\begin{array}{c}\text { Facilitators } \\
\text { Facilitators to Recommended Breastfeeding } \\
\text { Key Theme: Perceptions } \\
\end{array}$}} \\
\hline & & & & & & & & \\
\hline 59 & $\begin{array}{l}\text { Breastfeeding is inexpensive } \\
\text { and hygienic }\end{array}$ & $1 ; 2 ; 4 ; 7 ; 9 ; 13$ & $\begin{array}{l}\text { Moderate } \\
\text { methodological } \\
\text { limitations }\end{array}$ & $\begin{array}{l}\text { Minor concerns } \\
\text { about relevance }\end{array}$ & $\begin{array}{l}\text { Minor concerns } \\
\text { about coherence }\end{array}$ & $\begin{array}{l}\text { Minor concerns } \\
\text { about adequacy }\end{array}$ & High confidence & $\begin{array}{l}\text { This finding was graded as high confidence because of } \\
\text { minor concerns about relevance, coherence and } \\
\text { adequacy; and moderate methodological limitations. }\end{array}$ \\
\hline 60 & $\begin{array}{l}\text { Breastfeeding relieves } \\
\text { uncomfortable fullness } \\
\text { of breasts }\end{array}$ & 4 & $\begin{array}{l}\text { Moderate } \\
\text { methodological } \\
\text { limitations }\end{array}$ & NA & NA & $\begin{array}{l}\text { Moderate } \\
\text { concerns about } \\
\text { adequacy }\end{array}$ & Low confidence & $\begin{array}{l}\text { This finding was graded as low confidence because of } \\
\text { moderate concerns about adequacy and minimal studies } \\
\text { contributing evidence. }\end{array}$ \\
\hline 61 & $\begin{array}{l}\text { Breastfeeding strengthens the } \\
\text { bond between the mother } \\
\text { and child }\end{array}$ & $5 ; 9$ & $\begin{array}{l}\text { Moderate } \\
\text { methodological } \\
\text { limitations }\end{array}$ & $\begin{array}{l}\text { Moderate } \\
\text { concerns about } \\
\text { relevance }\end{array}$ & $\begin{array}{l}\text { Minor concerns } \\
\text { about coherence }\end{array}$ & $\begin{array}{l}\text { Moderate } \\
\text { concerns about } \\
\text { adequacy }\end{array}$ & $\begin{array}{l}\text { Moderate } \\
\text { confidence }\end{array}$ & $\begin{array}{l}\text { This finding was graded as moderate confidence because } \\
\text { of moderate concerns regarding methodology, relevance } \\
\text { and adequacy and minor concerns about coherence. }\end{array}$ \\
\hline \multicolumn{9}{|c|}{ Key Theme: Healthcare Services } \\
\hline 62 & $\begin{array}{l}\text { Advice and encouragement } \\
\text { provided in some healthcare } \\
\text { setting(s) (e.g., prenatal visits, } \\
\text { delivery, home visits) }\end{array}$ & $3 ; 10 ; 13 ; 16 ; 17 ; 18$ & $\begin{array}{l}\text { Moderate } \\
\text { methodological }\end{array}$ & $\begin{array}{l}\text { Minor concerns } \\
\text { about relevance }\end{array}$ & $\begin{array}{l}\text { Minor concerns } \\
\text { about coherence }\end{array}$ & $\begin{array}{l}\text { Substantial } \\
\text { concerns about } \\
\text { adequacy }\end{array}$ & $\begin{array}{l}\text { Moderate } \\
\text { confidence }\end{array}$ & $\begin{array}{l}\text { This finding was graded as moderate confidence because } \\
\text { of substantial concerns about adequacy, moderate } \\
\text { methodological limitations, minor concerns about } \\
\text { relevance and coherence. }\end{array}$ \\
\hline
\end{tabular}


Table 2. Cont

\begin{tabular}{|c|c|c|c|c|c|c|c|c|}
\hline $\begin{array}{l}\text { Finding } \\
\text { No. }\end{array}$ & Review Finding & $\begin{array}{l}\text { Studies } \\
\text { Contributing to the } \\
\text { Review Finding }\end{array}$ & $\begin{array}{l}\text { Assessment of } \\
\text { Methodological } \\
\text { Limitations }\end{array}$ & $\begin{array}{l}\text { Assessment of } \\
\text { Relevance }\end{array}$ & $\begin{array}{l}\text { Assessment of } \\
\text { Coherence }\end{array}$ & $\begin{array}{l}\text { Assessment of } \\
\text { Adequacy }\end{array}$ & $\begin{array}{l}\text { Overall CERQual } \\
\text { Assessment of } \\
\text { Confidence }\end{array}$ & Explanation of Judgement \\
\hline 63 & $\begin{array}{l}\text { Some advice and counseling } \\
\text { from health workers on } \\
\text { importance of breastfeeding }\end{array}$ & $\begin{array}{c}2 ; 3 ; 4 ; 9 ; 13 ; 14 ; 15 ; 16 ; \\
17 ; 18 ; 19\end{array}$ & $\begin{array}{l}\text { Moderate } \\
\text { methodological } \\
\text { limitations }\end{array}$ & $\begin{array}{l}\text { Minor concerns } \\
\text { about relevance }\end{array}$ & $\begin{array}{l}\text { Minor concerns } \\
\text { about coherence }\end{array}$ & $\begin{array}{l}\text { Substantial } \\
\text { concerns about } \\
\text { adequacy }\end{array}$ & $\begin{array}{l}\text { Moderate } \\
\text { confidence }\end{array}$ & $\begin{array}{l}\text { This finding was graded as moderate confidence because } \\
\text { of substantial concerns about adequacy, moderate } \\
\text { methodological limitations, minor concerns about } \\
\text { relevance and coherence. }\end{array}$ \\
\hline \multicolumn{9}{|c|}{ Key Theme: Infant-Specific Factors } \\
\hline 64 & $\begin{array}{l}\text { Child crying provides } \\
\text { guidance when to feed }\end{array}$ & $2 ; 10$ & $\begin{array}{l}\text { Minor } \\
\text { methodological } \\
\text { limitations }\end{array}$ & $\begin{array}{l}\text { Moderate } \\
\text { concerns about } \\
\text { relevance }\end{array}$ & $\begin{array}{l}\text { Minor concerns } \\
\text { about coherence }\end{array}$ & $\begin{array}{l}\text { Minor concerns } \\
\text { about adequacy }\end{array}$ & High confidence & $\begin{array}{l}\text { This finding was graded as high confidence due to only } \\
\text { minor concerns about adequacy, relevance and } \\
\text { methodological limitations and moderate concerns } \\
\text { about relevance. }\end{array}$ \\
\hline \multicolumn{9}{|c|}{ Key Theme: Mother-Specific Factors } \\
\hline 65 & $\begin{array}{l}\text { Mothers understand the } \\
\text { importance of breastfeeding }\end{array}$ & $\begin{array}{l}1 ; 2 ; 3 ; 4 ; 5 ; 7 ; 9 ; 10 ; 11 ; \\
15 ; 16 ; 17 ; 18 ; 19 ; 21 ; \\
22\end{array}$ & $\begin{array}{l}\text { Moderate } \\
\text { methodological } \\
\text { limitations }\end{array}$ & $\begin{array}{l}\text { Minor concerns } \\
\text { about relevance }\end{array}$ & $\begin{array}{l}\text { Minor concerns } \\
\text { about coherence }\end{array}$ & $\begin{array}{l}\text { Minor concerns } \\
\text { about adequacy }\end{array}$ & High confidence & $\begin{array}{l}\text { This finding was graded as high confidence because of } \\
\text { minor concerns about relevance, coherence and } \\
\text { adequacy; and moderate methodological limitations. }\end{array}$ \\
\hline 66 & $\begin{array}{l}\text { Mothers understand the } \\
\text { importance of colostrum }\end{array}$ & $2 ; 7 ; 14 ; 19$ & $\begin{array}{l}\text { Moderate } \\
\text { methodological } \\
\text { limitations }\end{array}$ & $\begin{array}{l}\text { Minor concerns } \\
\text { about relevance }\end{array}$ & $\begin{array}{l}\text { Minor concerns } \\
\text { about coherence }\end{array}$ & $\begin{array}{l}\text { Minor concerns } \\
\text { about adequacy }\end{array}$ & High confidence & $\begin{array}{l}\text { This finding was graded as high confidence because of } \\
\text { minor concerns about relevance, coherence and } \\
\text { adequacy; and moderate methodological limitations. }\end{array}$ \\
\hline \multicolumn{9}{|c|}{ Key Theme: Social and Cultural Factors } \\
\hline 67 & $\begin{array}{l}\text { Cultures and traditions } \\
\text { facilitate early initiation } \\
\text { of breastfeeding }\end{array}$ & 3 & $\begin{array}{l}\text { Moderate } \\
\text { methodological } \\
\text { limitations }\end{array}$ & NA & NA & $\begin{array}{l}\text { No concerns } \\
\text { about adequacy }\end{array}$ & Low confidence & $\begin{array}{l}\text { This finding was graded as low confidence because of } \\
\text { minimal studies contributing evidence. }\end{array}$ \\
\hline 68 & $\begin{array}{l}\text { Family support promotes } \\
\text { breastfeeding }\end{array}$ & $1 ; 2 ; 7 ; 9$ & $\begin{array}{l}\text { Moderate } \\
\text { methodological } \\
\text { limitations }\end{array}$ & $\begin{array}{l}\text { Minor concerns } \\
\text { about relevance }\end{array}$ & $\begin{array}{l}\text { Minor concerns } \\
\text { about coherence }\end{array}$ & $\begin{array}{l}\text { Substantial } \\
\text { concerns about } \\
\text { adequacy }\end{array}$ & $\begin{array}{l}\text { Moderate } \\
\text { confidence }\end{array}$ & $\begin{array}{l}\text { This finding was graded as moderate confidence because } \\
\text { of moderate methodological limitations, minor concerns } \\
\text { regarding relevance and coherence and substantial } \\
\text { concerns about adequacy. }\end{array}$ \\
\hline 69 & $\begin{array}{l}\text { Praise and affirmation from a } \\
\text { partner and/or family } \\
\text { facilitate breastfeeding }\end{array}$ & 2 & $\begin{array}{l}\text { Moderate } \\
\text { methodological } \\
\text { limitations }\end{array}$ & NA & NA & $\begin{array}{l}\text { No concerns } \\
\text { about adequacy }\end{array}$ & NA & NA \\
\hline \multicolumn{9}{|c|}{$\begin{array}{l}\text { Facilitators to Complementary Feeding } \\
\text { Kev Theme: Food Security }\end{array}$} \\
\hline 70 & $\begin{array}{l}\text { Some healthy foods } \\
\text { are available }\end{array}$ & $16 ; 18 ; 22$ & $\begin{array}{l}\text { Moderate } \\
\text { methodological } \\
\text { limitations }\end{array}$ & $\begin{array}{l}\text { Key } \\
\text { Moderate } \\
\text { concerns about } \\
\text { relevance }\end{array}$ & $\begin{array}{l}\text { Theme: Food Secur } \\
\text { Minor concerns } \\
\text { about coherence }\end{array}$ & $\begin{array}{l}\text { Minor concerns } \\
\text { about adequacy }\end{array}$ & $\begin{array}{l}\text { Moderate } \\
\text { confidence }\end{array}$ & $\begin{array}{l}\text { This finding was graded as moderate confidence because } \\
\text { of moderate concerns about methodology and relevance; } \\
\text { and minor concerns about coherence and adequacy. }\end{array}$ \\
\hline \multicolumn{9}{|c|}{ Key Theme: Social Support } \\
\hline 71 & $\begin{array}{l}\text { Family support promotes } \\
\text { complementary feeding }\end{array}$ & $6 ; 15 ; 16 ; 21$ & $\begin{array}{l}\text { Moderate } \\
\text { methodological } \\
\text { limitations }\end{array}$ & $\begin{array}{l}\text { Moderate } \\
\text { concerns about } \\
\text { relevance }\end{array}$ & $\begin{array}{l}\text { Minor concerns } \\
\text { about coherence }\end{array}$ & $\begin{array}{l}\text { Minor concerns } \\
\text { about adequacy }\end{array}$ & $\begin{array}{l}\text { Moderate } \\
\text { confidence }\end{array}$ & $\begin{array}{l}\text { This finding was graded as moderate confidence because } \\
\text { of minor concerns about coherence and adequacy; and } \\
\text { moderate concerns regarding relevance } \\
\text { and methodology. }\end{array}$ \\
\hline
\end{tabular}


Table 2. Cont

\begin{tabular}{|c|c|c|c|c|c|c|c|c|}
\hline $\begin{array}{l}\text { Finding } \\
\text { No. }\end{array}$ & Review Finding & $\begin{array}{c}\text { Studies } \\
\text { Contributing to the } \\
\text { Review Finding }\end{array}$ & $\begin{array}{c}\text { Assessment of } \\
\text { Methodological } \\
\text { Limitations }\end{array}$ & $\begin{array}{l}\text { Assessment of } \\
\text { Relevance }\end{array}$ & $\begin{array}{l}\text { Assessment of } \\
\text { Coherence }\end{array}$ & $\begin{array}{l}\text { Assessment of } \\
\text { Adequacy }\end{array}$ & $\begin{array}{l}\text { Overall CERQual } \\
\text { Assessment of } \\
\text { Confidence }\end{array}$ & Explanation of Judgement \\
\hline 72 & $\begin{array}{l}\text { Mothers understand the } \\
\text { importance of } \\
\text { complementary feeding }\end{array}$ & $15 ; 16 ; 18$ & $\begin{array}{l}\text { Moderate } \\
\text { methodological } \\
\text { limitations }\end{array}$ & $\begin{array}{l}\text { Moderate } \\
\text { concerns about } \\
\text { relevance }\end{array}$ & $\begin{array}{l}\text { Minor concerns } \\
\text { about coherence }\end{array}$ & $\begin{array}{l}\text { Minor concerns } \\
\text { about adequacy }\end{array}$ & $\begin{array}{c}\text { Moderate } \\
\text { confidence }\end{array}$ & $\begin{array}{l}\text { This finding was graded as moderate confidence because } \\
\text { of minor concerns about coherence and adequacy; and } \\
\text { moderate concerns. }\end{array}$ \\
\hline \multicolumn{9}{|c|}{ Key Theme: Healthcare Services } \\
\hline 73 & $\begin{array}{l}\text { Mothers receive IYCF advice } \\
\text { and counseling at } \\
\text { healthcare facilities }\end{array}$ & $15 ; 18 ; 19 ; 21$ & $\begin{array}{l}\text { Moderate } \\
\text { methodological }\end{array}$ & $\begin{array}{l}\text { Minor concerns } \\
\text { about relevance }\end{array}$ & $\begin{array}{l}\text { Minor concerns } \\
\text { about coherence }\end{array}$ & $\begin{array}{l}\text { Substantial } \\
\text { concerns about } \\
\text { adequacy }\end{array}$ & $\begin{array}{l}\text { Moderate } \\
\text { confidence }\end{array}$ & $\begin{array}{l}\text { This finding was graded as moderate confidence because } \\
\text { of minor concerns about coherence and adequacy; and } \\
\text { moderate methodological limitations and substantial } \\
\text { concerns about adequacy. }\end{array}$ \\
\hline
\end{tabular}

NA: not applicable. 
Following abstraction and grading of evidence, the final step involved data from the results, discussion, and conclusion sections of the included studies, being extracted and entered into NVivo 11 qualitative software (QSR International Pty Ltd., Version 11, 2015, Burlington, MA, USA), wherein thematic analysis was employed to identify domains descriptive of the data for investigation and presentation.

This thematic analysis led to a more interpretive phase in order to understand how the themes identified may represent barriers and facilitators to change infant and young child feeding; for this we used an approach similar to that of Thomas et al. [24].

\section{Results}

After a narrative summary of themes identified through the initial analysis was available, three reviewers met jointly to consider these and produce a consensus-based listing of barriers and facilitators. This step evaluated all emergent findings in light of the summary of themes wherein several emergent findings were often merged into one synthesized finding, or individual emergent findings were discarded due to weak assessments of confidence or minimal support from the included studies. Table 3 presents the synthesized review findings of barriers and facilitators generated through the data synthesis exercise and subsequently agreed on by reviewers.

Four categories of barriers to recommended breastfeeding practices were identified, and three categories of barriers to recommended complementary feeding practices were identified. None of the studies included in the final review disclosed funding from food manufacturers, nor disclosed any related conflicts of interests. Barriers to breastfeeding included factors specific to infant or mother, and cross-cutting beliefs and perceptions, as well as a pervasive lack of support for breastfeeding, from families, health workers and due to time poverty. Several categories of facilitators were also identified from the literature reviewed, including food security, social support, and individual infant and maternal factors. 
Table 3. CERQual summary of qualitative review findings.

\begin{tabular}{|c|c|c|c|c|}
\hline Objective: & \multicolumn{4}{|c|}{$\begin{array}{l}\text { To systematically review qualitative literature related to family experiences (particularly parental ones) of infant and young child feeding in low-income and lower-middle income countries, synthesizing } \\
\text { information on the barriers and facilitators that may relate to interventions to impact nutrition, survival, growth and development. }\end{array}$} \\
\hline Perspective: & \multicolumn{4}{|c|}{ Experiences, beliefs, attitudes and perceptions of parents/caregivers who routinely engage in infant and young child feeding. } \\
\hline Included studies: & \multicolumn{4}{|c|}{$\begin{array}{c}\text { Included studies involved participants from low-income or lower-middle income countries who were parents or close family members of an infant or young child (0-2 years of age) at the time of the study } \\
\text { used widely accepted qualitative data collection methods; and provided a clear description of recognized qualitative data analysis methods. }\end{array}$} \\
\hline Key theme & Synthesized Review Finding & $\begin{array}{l}\text { Emergent findings incorporated into } \\
\text { synthesized finding (finding numbers } \\
\text { from Table 2) }\end{array}$ & $\begin{array}{l}\text { Studies contributing evidence for } \\
\text { review finding (study numbers from } \\
\text { Table 1) }\end{array}$ & Overall CERQual assessment of confidence \\
\hline \multirow{2}{*}{\multicolumn{5}{|c|}{$\begin{array}{c}\text { Barriers } \\
\text { Barriers to Recommended Breastfeeding Practices }\end{array}$}} \\
\hline & & & & \\
\hline \multirow{6}{*}{ Beliefs and perceptions } & Breastfeeding alters a woman's breasts in an undesirable way & 1 & $1 ; 4 ; 13$ & High confidence \\
\hline & $\begin{array}{l}\text { Traditional beliefs indicate that colostrum is not good for the baby, or } \\
\text { that breastfeeding should be delayed until more white milk comes in } \\
\text { • Colostrum is not healthy for baby, so mothers discard it } \\
\text { - Delay in breastmilk "coming in" following the birth (delayed onset of } \\
\text { Lactogenesis II) }\end{array}$ & $7 ; 13$ & $3 ; 7 ; 10 ; 18$ & Moderate confidence \\
\hline & $\begin{array}{l}\text { The breastmilk produced by mother is not sufficient for the infant } \\
\text { • not enough water or sustenance in the breastmilk } \\
\text { • babies are thirsty and need water } \\
\text { • baby still cries even after mother breastfeeds (so caregivers believe } \\
\text { baby must still be hungry or thirsty) } \\
\text { • mothers do not have confidence that they are producing sufficient } \\
\text { breast milk to meet baby's needs }\end{array}$ & $5 ; 8 ; 10 ; 11 ; 15 ; 16$ & $\begin{aligned} 2 ; 3 ; 4 ; 5 ; 6 ; 7 ; 8 ; 9 ; 13 ; 14 ; 15 ; 16 ; 17 ; 19 ; \\
21 ; 23\end{aligned}$ & Moderate/High confidence \\
\hline & $\begin{array}{l}\text { Traditional foods/drinks are believed to be good for baby's health and } \\
\text { encouraged by older influential family members, undermining } \\
\text { exclusive breastfeeding }\end{array}$ & $12 ; 14 ; 17 ; 18 ; 27 ; 28$ & $2 ; 7 ; 11 ; 14 ; 16 ; 17 ; 18 ; 19 ; 20 ; 21 ; 22$ & Moderate/High confidence \\
\hline & $\begin{array}{l}\text { Women should cease breastfeeding if they become pregnant before } \\
\text { nursling is } 2 \text { years old }\end{array}$ & 20 & $15 ; 17 ; 18 ; 21$ & Moderate confidence \\
\hline & $\begin{array}{l}\text { Breast milk substitutes can make a baby healthier/Media has a } \\
\text { negative impact on breastfeeding (promoting breastmilk substitutes as } \\
\text { healthy for babies) }\end{array}$ & $9 ; 26$ & $7 ; 8 ; 13$ & High confidence \\
\hline Lack of support & $\begin{array}{l}\text { Family, peers or close neighbors do not support, or undermine optimal } \\
\text { breastfeeding practices: } \\
\text { • Family members do not support ideal breastfeeding practices } \\
\text { - Traditional beliefs and influence of older female family members } \\
\text { (e.g., grandmothers) influence breastfeeding } \\
\text { - Comments/advice from families, peers or close neighbors influence } \\
\text { breastfeeding }\end{array}$ & $21 ; 27 ; 28$ & $4 ; 8 ; 9 ; 13 ; 19 ; 16 ; 18 ; 20 ; 21$ & Moderate/High confidence \\
\hline
\end{tabular}


Table 3. Cont

\begin{tabular}{|c|c|c|c|c|}
\hline & $\begin{array}{l}\text { Lack of support from HWs } \\
\bullet \text { HWs do not reassure women about their ability to breastfeed } \\
\bullet \text { HWs do not have adequate knowledge and skills to support mothers } \\
\bullet \text { Mothers do not receive advice from HWs }\end{array}$ & $23 ; 24 ; 25$ & $2 ; 3 ; 9 ; 10 ; 13 ; 23$ & Moderate/High confidence \\
\hline & $\begin{array}{l}\text { Mothers do not have enough time to breastfeed their infants due } \\
\text { to work } \\
\text { • maternity leave is short } \\
\text { - minimal acceptance of expressing breastmilk } \\
\text { - unwillingness to breastfeed in public-not wanting to expose breasts }\end{array}$ & 22 & $4 ; 6 ; 7 ; 8 ; 9 ; 13 ; 14 ; 15 ; 16 ; 17$ & Moderate confidence \\
\hline Infant-specific factors & $\begin{array}{l}\text { Baby stops or is unable to breastfeed: } \\
\bullet \text { baby has an illness } \\
\text { • babies stop breastfeeding for unknown reasons (lack of interest in } \\
\text { feeding, self-weaning) }\end{array}$ & $29 ; 30 ; 31$ & $9 ; 4 ; 10 ; 13 ; 14 ; 17 ; 19$ & Moderate confidence \\
\hline \multirow[t]{4}{*}{ Mother-specific factors } & $\begin{array}{l}\text { Lack of knowledge and education about breastfeeding which } \\
\text { influences her self confidence } \\
\text { - Mothers do not understand purpose of colostrum and } \\
\text { breastfeeding initiation } \\
\text { - Mothers do not have knowledge about breastfeeding } \\
\text { practices generally } \\
\text { - Mothers do not have formal schooling or education } \\
\end{array}$ & $32 ; 33 ; 35$ & $\begin{array}{r}2 ; 3 ; 7 ; 8 ; 9 ; 10 ; 13 ; 14 ; 15 ; 17 ; 18 ; 20 ; 21 ; \\
22\end{array}$ & Moderate confidence \\
\hline & Mothers unable to interpret child's feeding cues or behavior & 34 & $8 ; 17 ; 19$ & Moderate confidence \\
\hline & $\begin{array}{l}\text { Mother's health problems influence breastfeeding: } \\
\text { - illness } \\
\text { - death } \\
\text { - breast or nipple problems (swollen and painful breasts, sore nipples, } \\
\text { breast abscesses) } \\
\text { - childbirth complications (causing delay in initiation of breastfeeding) }\end{array}$ & $36 ; 37 ; 38$ & $3 ; 4 ; 5 ; 7 ; 9 ; 10 ; 13 ; 14 ; 15 ; 17 ; 18 ; 19 ; 21$ & Moderate/High confidence \\
\hline & $\begin{array}{l}\text { Mothers attribute their inability to produce enough breastmilk to their } \\
\text { own diet }\end{array}$ & 19 & $4 ; 11 ; 13 ; 14 ; 17 ; 18 ; 21 ; 23$ & High confidence \\
\hline \multicolumn{5}{|c|}{ Barriers to Recommended Complementary Feeding Practices } \\
\hline \multirow{4}{*}{$\begin{array}{l}\text { Food security and social } \\
\text { and cultural factors }\end{array}$} & $\begin{array}{l}\text { Household food insecurity; } \\
\text { - replacement of higher quality foods with lower quality foods for } \\
\text { financial reasons or drought }\end{array}$ & $39 ; 40$ & $11 ; 12 ; 14 ; 15 ; 16 ; 17 ; 20 ; 21 ; 23$ & High confidence \\
\hline & Lack of diversity in available foods; food shortages & 41 & $12 ; 14 ; 15 ; 16 ; 17 ; 19 ; 20$ & High confidence \\
\hline & Lack of water for cooking & 42 & $12 ; 15$ & Moderate confidence \\
\hline & $\begin{array}{l}\text { Mothers lack financial decision making power, inhibiting ability to } \\
\text { buy food }\end{array}$ & 45 & $14 ; 20$ & Moderate confidence \\
\hline
\end{tabular}


Table 3. Cont.

\begin{tabular}{|c|c|c|c|c|}
\hline \multirow[t]{3}{*}{ Infant-specific factors } & $\begin{array}{l}\text { Animal source foods are not suitable for infants: } \\
\text { - infants do not like animal source foods } \\
\text { - infants vomit when given animal source foods } \\
\text { - infants lack appetite for animal source foods } \\
\text { - animal source foods are not appropriate for infants }\end{array}$ & $46 ; 47 ; 49$ & $6 ; 15 ; 18 ; 23$ & Low/Moderate confidence \\
\hline & Infants refuse to eat and or spit out foods & 48 & $6 ; 15 ; 23$ & Low confidence \\
\hline & Infants have an illness & 50 & $15 ; 18$ & Moderate confidence \\
\hline \multirow{3}{*}{ Family factors } & $\begin{array}{l}\text { Mothers and other family members do not have appropriate } \\
\text { knowledge about complementary feeding }\end{array}$ & 51 & $6 ; 11 ; 14 ; 15 ; 16 ; 19 ; 20 ; 21 ; 23$ & High confidence \\
\hline & $\begin{array}{l}\text { Mother and other family members do not feel that baby can eat } \\
\text { solid foods } \\
\text { • fear of choking (e.g., on meat) } \\
\text { - thicker weaning porridge believed to be difficult for young children } \\
\text { to swallow/digest } \\
\text { • lack of knowledge and self confidence in how to make foods } \\
\text { appropriate for young child }\end{array}$ & $53 ; 55$ & $15 ; 16 ; 19 ; 20 ; 23$ & Moderate confidence \\
\hline & Lack of time to cook special foods for the child & 56 & $15 ; 16$ & Moderate confidence \\
\hline \multirow{2}{*}{\multicolumn{5}{|c|}{$\begin{array}{l}\text { Facilitators } \\
\text { mmended Breastfeeding Practices }\end{array}$}} \\
\hline & & & & \\
\hline \multirow[t]{2}{*}{ Perceptions } & $\begin{array}{l}\text { Breastfeeding is good for baby: makes baby grow strong, healthy and } \\
\text { intelligent, prevents and shortens duration of illness, etc. }\end{array}$ & 65 & $\begin{array}{c}1 ; 2 ; 3 ; 4 ; 5 ; 7 ; 9 ; 10 ; 11 ; 15 ; 16 ; 17 ; 18 ; \\
19 ; 21 ; 22\end{array}$ & High confidence \\
\hline & Breastfeeding is inexpensive and hygienic & 59 & $1 ; 2 ; 4 ; 7 ; 9 ; 13$ & High confidence \\
\hline Healthcare services & $\begin{array}{l}\text { Advice and encouragement provided in some healthcare setting(s) } \\
\text { (e.g., prenatal visits, delivery, home visits); Some advice and } \\
\text { counseling from health workers on importance of breastfeeding }\end{array}$ & $62 ; 63$ & $2 ; 3 ; 4 ; 9 ; 10 ; 13 ; 14 ; 15 ; 16 ; 17 ; 18 ; 19$ & Moderate confidence \\
\hline Infant-specific factors & Child crying provides guidance when to feed & 64 & $2 ; 10$ & High confidence \\
\hline \multirow{3}{*}{ Mother-specific factors } & Breastfeeding relieves the fullness of mothers' breasts & 60 & 4 & Low confidence \\
\hline & Breastfeeding strengthens mothers bond with infant & 61 & $5 ; 9$ & Moderate confidence \\
\hline & Mother understands the importance of colostrum & 66 & $2 ; 7 ; 14 ; 19$ & High confidence \\
\hline \multirow{2}{*}{ Social and cultural factors } & Cultures and traditions facilitate early initiation of breastfeeding & 67 & 3 & Low confidence \\
\hline & Family supports recommended breastfeeding practices & $68 ; 69$ & $1 ; 2 ; 7 ; 9$ & Moderate confidence \\
\hline \multicolumn{5}{|c|}{ Facilitators to Recommended Complementary Feeding Practices } \\
\hline Food security & Some healthy foods are available & 70 & $16 ; 18 ; 22$ & Moderate confidence \\
\hline Social support & Family supports recommended complementary feeding practices & 71 & $6 ; 15 ; 16 ; 21$ & Moderate confidence \\
\hline Mother-specific factors & $\begin{array}{l}\text { Mother knows the importance of proper complementary } \\
\text { feeding practices }\end{array}$ & 72 & $15 ; 16 ; 18$ & Moderate confidence \\
\hline Healthcare services & Mothers receive IYCF advice and counseling at healthcare facilities & 73 & $15 ; 18 ; 19 ; 21$ & Moderate confidence \\
\hline
\end{tabular}




\section{Conclusions}

The findings contribute to the expanding literature on family experiences related to breastfeeding and complementary feeding of young children and infants in low-income settings and constitute the most comprehensive summary of findings to date. Previous systematic reviews of qualitative literature related to infant feeding have not included as broad an approach to the topic as the current review, having focused rather on specific areas such as bottle feeding [25] and obesogenic dietary intake. [26] Another qualitative review, undertaken in 2008, focused solely on maternal support for breastfeeding mothers [27] and one carried out in 2013 considered the psychosocial correlates of exclusive breastfeeding [28]. Neither of those included a comprehensive approach to assessing the quality of included studies.

Strengths of the current review included the use of multiple reviewers experienced in qualitative research and data collection and analysis, a comprehensive search strategy, assessment and scoring of quality and confidence placed in the findings based on guidelines, and inclusion of grey literature. Limitations of the study were the exclusion of documents not available in the English language and date limitations. Two authors of the present review, who conduct research in this topical area, have authored or co-authored articles included in the review, which inclusion was based solely on having met the eligibility criteria.

Overall, this review focused on identifying qualitative and ethnographic studies that related the experiences and first-hand accounts of family members responsible for providing for the care and nutritional needs of young children under 2 years of age. Through this systematic qualitative review and synthesis, hypothesized barriers and facilitators to improving infant and young child feeding have been identified. The findings presented in this review are directly applicable to social and behavioral change initiatives in low resource settings aimed at improving dietary patterns and practices for better health and nutrition of young children.

Supplementary Materials: The following are available online at www.mdpi.com/2072-6643/9/10/1140/s1, Table S1: ENTREQ checklist (Enhancing transparency in reporting the synthesis of qualitative research).

Acknowledgments: No funding was provided for this study. All authors have read and approved the final manuscript.

Author Contributions: A.N.B. conceived and designed the study. All authors jointly contributed to the implementation of the research, to the analysis of the results, and to the writing of the manuscript.

Conflicts of Interest: All authors declare that they have no competing interests.

\section{Appendix A}

Details of search strategy.

\begin{tabular}{|c|c|c|c|}
\hline Database & $\begin{array}{l}\text { Date of } \\
\text { Search }\end{array}$ & Search Terms & $\begin{array}{c}\text { Total Articles } \\
\text { Returned }\end{array}$ \\
\hline Pubmed & 9 July 2016 & $\begin{array}{l}\text { "infant nutritional physiological phenomena" (MeSH Terms) AND “qualitative } \\
\text { research" (MeSH Terms) AND ("13 July 2006" (PDat): “9 July 2016" (PDat) AND } \\
\text { "humans" (MeSH Terms) AND English (lang)) }\end{array}$ & 180 articles \\
\hline Ebsco & 9 July 2016 & $\begin{array}{c}\text { ((MH “Infant Nutritional Physiology+”) OR (MH “Infant Feeding+”)) AND } \\
\text { Qualitative studies } \\
\text { Limiters-Published Date: } 1 \text { January 2006-31 December 2016; English Language; } \\
\text { Abstract Available; Human; Language: English } \\
\text { Search modes-Find all my search terms }\end{array}$ & 287 articles \\
\hline Embase & 9 July 2016 & $\begin{array}{l}\text { 'infant feeding'/exp OR 'infant feeding' OR 'complementary feeding'/exp OR } \\
\text { 'complementary feeding' AND ('qualitative research' / exp OR 'qualitative } \\
\text { research') AND (embase)/lim AND (2006-2016)/py AND (humans)/lim }\end{array}$ & 151 articles \\
\hline
\end{tabular}




\section{Appendix B}

\begin{tabular}{|c|c|c|c|c|}
\hline $\begin{array}{l}\text { Study } \\
\text { No. }\end{array}$ & Author and Title & Participant Characteristics & Key Outcomes Reported & $\begin{array}{l}\text { Emergent Findings } \\
\text { (Finding Numbers } \\
\text { from Table 2) }\end{array}$ \\
\hline \multicolumn{5}{|c|}{ Studies Identified from Search Databases } \\
\hline 1 & $\begin{array}{l}\text { Lynn M. Babington } \\
\text { Understanding Beliefs, Knowledge, } \\
\text { and Practices of Mothers in the } \\
\text { Dominican Republic Related to } \\
\text { Feeding Infants and Young Children }\end{array}$ & 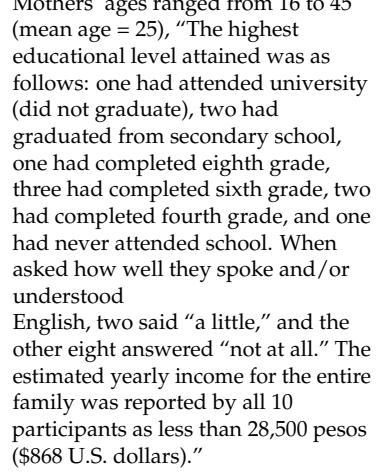 & $\begin{array}{l}\text { From abstract: "The women who participated in the focus group all breast fed their babies with the belief that breast milk } \\
\text { is better for infants than formula. Cereal, fruits, and vegetables are the main diet for children under } 3 \text { years old. Typically, } \\
\text { "fast foods" and snack foods are not readily available and therefore they are rarely consumed. Obesity was viewed as a } \\
\text { health risk for children. These mothers were practicing many healthy feeding behaviors, such as breastfeeding and } \\
\text { limiting fats and sweets for young children, and they recognized their importance in promoting the health of children." } \\
\text { Additional notes: Participants universally practiced breastfeeding in part because formula is expensive and not readily } \\
\text { available. They recognized the association between breastfeeding and the health of their babies. They all learned to } \\
\text { breastfeed from their mothers/grandmother/sisters and said that everyone in the DR learns this way. Older mothers } \\
\text { mentioned that breastfeeding has a negative effect on the physical appearance of their breasts but that this did not impact } \\
\text { their decision to breastfeed because formula is not affordable. } \\
\text { Mothers said they avoid giving fast foods and snack foods (which are not readily available), greasy / fatty foods, and } \\
\text { sweats to young children. Since they do not give greasy /fatty foods to young children, they do not give much meat until } \\
\text { around } 3 \text { years because it is greasy. Participants said that } 3 \text { years is the age when children eat what everyone else in the } \\
\text { house eats in the DR. }\end{array}$ & $\begin{array}{l}(1) \\
(59) \\
(655) \\
(68)\end{array}$ \\
\hline 2 & $\begin{array}{l}\text { Amal Omer-Salim, Lars-Ake Persson, } \\
\text { Pia Olsson } \\
\text { Whom can I rely on? Mothers' } \\
\text { approaches to support for feeding: } \\
\text { An interview study in suburban Dar } \\
\text { es Salaam, Tanzania }\end{array}$ & $\begin{array}{l}\text { Mothers were aged 16- } 30 \text { years. Four } \\
\text { women had no formal education, two } \\
\text { had completed primary education } \\
\text { and two had completed secondary } \\
\text { education. Five of the women were } \\
\text { homemakers and three were either } \\
\text { formally employed or self-employed }\end{array}$ & $\begin{array}{l}\text { From abstract: “The study revealed four categories of mothers' perceptions of baby feeding: (1) baby feeding, housework } \\
\text { and paid work have to adjust to each other; (2) breast feeding has many benefits; (3) water or breast milk can be given to } \\
\text { quench baby's thirst; and (4) crying provides guidance for baby feeding. Four different themes describing approaahes to } \\
\text { support emerged from the data: (1) adhering to diverse sources; (2) relying wholeheartedly on a mother figure; (3) } \\
\text { working as a parental team; and (4) making arrangements for absence from the child.” } \\
\text { Additional notes: Mothers believed breastfeeding makes babies healthy and prevents diseases. Also, breastffeeding was } \\
\text { viewed as a cheap and hygienic option. Some believe colostrum is good for the baby and so initiated breastfeeding soon } \\
\text { after birth, but some women did not believe this and discard it. Good nutrition for the mother thought to improve } \\
\text { breastmilk output. Some women practice exclusive breastffeeding believing that breastmilk is all a baby needs, but some } \\
\text { think the baby also needs water (but they know that the water must be clean to give to baby). It is a traditional practice to } \\
\text { give boiled water shortly after birth. Some believe that giving water relieves baby from constipation. Initial crying was } \\
\text { recognized as a prompt for breastfeeding, but too much crying led mother to give water or porridge and was seen as a } \\
\text { sign of hunger (traditional beliefs say porridge will solve this and baby will stop crying). } \\
\text { Advice from HWs was sometimes inadequate (too general, or infeasible for mother). Advice from } \\
\text { mother /mother-in-law/senior mother figure sometimes refutes the advice given by HWs. Support and agreement from } \\
\text { the husband encourages breastfeeding. Praise from partner or HW encouraged mother to continue to exclusively } \\
\text { breastfeed. } \\
\text { Work interrupted optimal baby feeding for many mothers, but some mothers reported expressing breastmilk into a clean } \\
\text { cup while working to continue to breastfeed the baby. }\end{array}$ & $\begin{array}{l}(11) \\
(18) \\
(24) \\
(35) \\
(59) \\
(63) \\
(64) \\
(65) \\
(66) \\
(68) \\
(69)\end{array}$ \\
\hline
\end{tabular}




\begin{tabular}{|c|c|c|c|c|}
\hline $\begin{array}{c}\text { Study } \\
\text { No. }\end{array}$ & Author and Title & Participant Characteristics & Key Outcomes Reported & $\begin{array}{l}\text { Emergent Findings } \\
\text { (Finding Numbers } \\
\text { from Table 2) }\end{array}$ \\
\hline 3 & $\begin{array}{l}\text { C Tawiah-Agyemang, BR Kirkwood, } \\
\text { K Edmond, A Bazzano and Z Hill } \\
\text { Early initiation of breast-feeding in } \\
\text { Ghana: barriers and facilitators }\end{array}$ & $\begin{array}{l}\text { Of the } 52 \text { recently delivered mothers: } \\
\text { age ranged from under } 20 \text { to } 45 \text {. The } \\
\text { majority of them lived in rural areas } \\
(n=43,83 \%) \text {. Nearly half the mothers } \\
\text { did not complete any education } \\
(n=24,46 \%) ; 10 \text { attained primary } \\
\text { school; and } 18 \text { had attained education } \\
\text { beyond primary school. }\end{array}$ & $\begin{array}{l}\text { From abstract: "The major reasons for delaying initiation of breast-feeding were the perception of a lack of breast milk, } \\
\text { performing postbirth activities such as bathing, perception that the mother and the baby need rest after birth and the } \\
\text { baby not crying for milk. Facilitating factors for early initiation included delivery in a health facility, where the staff } \\
\text { encouraged early breastfeeding, and the belief in some ethnic groups that putting the baby to the breast encourages the } \\
\text { milk.". } \\
\text { Additional notes: Barriers for delayed initiation: } \\
\text { The perception of a lack of breastmilk: including physical signs that milk is absent/insufficient, beliefs about colostrum, } \\
\text { and traditional beliefs of when the milk comes in (believed to be on the third day for some cultural groups in the study). } \\
\text { Some believed colostrum was not good for the baby and so discarded it or delayed initiation until white milk came in. } \\
\text { Delayed initiation of breastfeeding beyond } 12 \mathrm{~h} \text { often led to prelacteal feeding. } \\
\text { Facilitators: Delivery at a health facility facilitated early initiation of breastfeeding due to advice of HWs. }\end{array}$ & $\begin{array}{l}(7) \\
(10) \\
(13) \\
(23) \\
(25) \\
(32) \\
(38) \\
(62) \\
(63) \\
(65) \\
(67)\end{array}$ \\
\hline 4 & $\begin{array}{l}\text { Gloria E. Otoo, Anna A. Lartey, } \\
\text { Rafael Pérez-Escamilla } \\
\text { Perceived Incentives and Barriers to } \\
\text { Exclusive Breastfeeding Among } \\
\text { Peri-urban Ghanaian Women }\end{array}$ & $\begin{array}{l}\text { The ages of the } 35 \text { participants ranged } \\
\text { from } 19 \text { to } 49 \text { (mean age was } 27.5) \text {. } \\
\text { They had } 7.2 \pm 3.6 \text { years of } \\
\text { formal education. } \\
\text { All but } 3 \text { of the women were } \\
\text { employed, with about half }(n=17) \\
\text { being traders. }\end{array}$ & 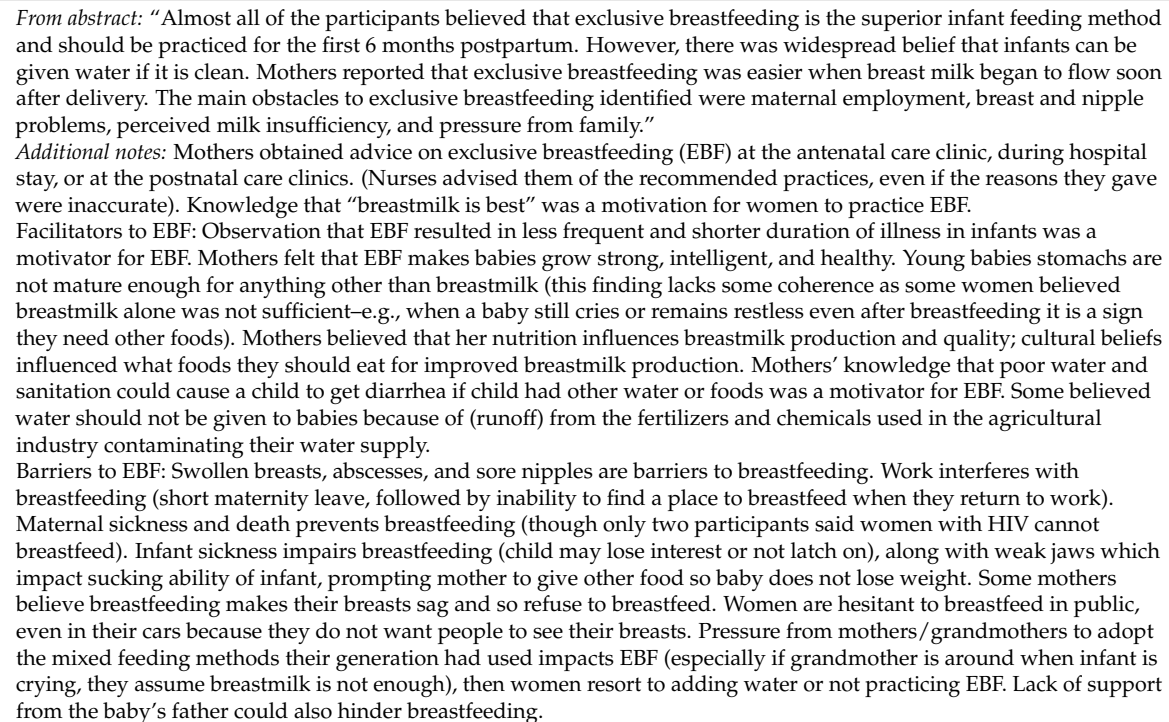 & $\begin{array}{l}(1) \\
(6) \\
(10) \\
(21) \\
(22) \\
(31) \\
(36) \\
(37) \\
(59) \\
(60) \\
(63) \\
(65)\end{array}$ \\
\hline
\end{tabular}




\begin{tabular}{|c|c|c|c|c|}
\hline $\begin{array}{l}\text { Study } \\
\text { No. }\end{array}$ & Author and Title & Participant Characteristics & Key Outcomes Reported & $\begin{array}{l}\text { Emergent Findings } \\
\text { (Finding Numbers } \\
\text { from Table 2) }\end{array}$ \\
\hline 5 & $\begin{array}{l}\text { Luzmil A Hernandez, } \\
\text { Marth A Lucia Vasqu } \\
\text { Practices and beliefs about exclusive } \\
\text { breastfeeding by women living in } \\
\text { Commune } 5 \text { in Cali, Colombia }\end{array}$ & $\begin{array}{l}\text { The women participating ranged } \\
\text { from } 20 \text { to } 33 \text { years of age. Regarding } \\
\text { the level of education, one of the } \\
\text { women had done university studies, } \\
\text { four had completed high school, } 5 \\
\text { had not finished high school, and the } \\
\text { remaining participants had been in } \\
\text { grammar School. }\end{array}$ & $\begin{array}{l}\text { From abstract: "Findings are presented in two parts: practices and beliefs in favor of exclusive breastfeeding and practices } \\
\text { and beliefs that do not support exclusive breastfeeding. The prominent practices and beliefs in favor of exclusive } \\
\text { breastfeeding are related to the mother's bond with the child, preparation for breastfeeding during pregnancy, and family } \\
\text { support. Among the practices and beliefs not supporting maternal breastfeeding, we must highlight the mother's lack of } \\
\text { confidence in her breast milk production." } \\
\text { Addititional notess: Breastfeeding was associated with providing love to their child and strengthening their bond. Women } \\
\text { were convinined that breastfed children will be healthier and smarter in the future. But, exclusive breastfeeding for six } \\
\text { months was considered difficult, and required preparation. They want their milk to be of adequate quality and quantity } \\
\text { for their child. Some grandmothers or other elders had ideas of ways mothers could improves these milk traits (e.g., } \\
\text { consuming fennel water). Mothers, mothers-in-law and grandmothers helped prepare mothers to breastfeed through } \\
\text { their own experienceses Some still felt that breastmilk alone would not be enough for their child. Especially if the child } \\
\text { continued crying. Pain in the breast was another reason for not exclusively breastfeeding. Some mothers who exclusively } \\
\text { breastfed reported their child had become dependent on the breast and it was then difficult to wean. Along this line, } \\
\text { mothers believed that weaning would be easier if the child had already had milk from other sources. } \\
\text { There is a belief that children are born with weak stomachs and something must be done to strengthen it and protect } \\
\text { them from disease. Home preparations are often given tot eh child within days after birth for this purpose; these } \\
\text { included bean tincture, bacon, or a liquid to purge the stomach. }\end{array}$ & $\begin{array}{l}(8) \\
(10) \\
(15) \\
(36) \\
(61) \\
(65)\end{array}$ \\
\hline 6 & $\begin{array}{l}\text { Sabrina Rasheed, Rukhsana Haider, } \\
\text { Nazmul Hassan, Helena Pachon, } \\
\text { Sanjeeda Islam, Chowdhury S.B. Jalal, } \\
\text { Tina G. Sanghvi } \\
\text { Why does nutrition deteriorate } \\
\text { rapidly among children under } 2 \text { years } \\
\text { of age? Using qualitative methods to } \\
\text { understand community perspectives } \\
\text { on complementary feeding practices } \\
\text { in Bangladesh }\end{array}$ & Not stated & $\begin{array}{l}\text { From abstract: "Lay perceptions about complementary feeding differ substantially from international complementary } \\
\text { feeding recommendations. A large proportion of children do not consume sufficient amounts of complementary foods to } \\
\text { meet their energy and micronutrient needs. } \\
\text { There was a agp in knowledge about appropriate complementary foods in terms of quality and quantity and strategies to } \\
\text { convert family foods to make them suitable for children. Complementary feeding advice from family members, peers, } \\
\text { and health workers, the importance given to feeding young children, and time spent by caregivers in feeding influenced } \\
\text { the timing, frequency, types of food given, and ways in which complementary feeding occurred." } \\
\text { Additional notes: Children were fed complementary foods prior to } 6 \text { months of age primarily due to the perception that } \\
\text { breastmilk alone was inadequate as the child got older. Liquids were given to young babies, including formula, which } \\
\text { was considered good to maintain the health of the baby. }\end{array}$ & $\begin{array}{l}(8) \\
(10) \\
(22) \\
(46) \\
(47) \\
(48) \\
(49) \\
(51) \\
(71)\end{array}$ \\
\hline 7 & $\begin{array}{l}\text { Pranee C. Lundberg, } \\
\text { Trieu Thi Ngoc Thu } \\
\text { Breast-feeding attitudes and practices } \\
\text { among Vietnamese mothers in Ho } \\
\text { Chi Minh City }\end{array}$ & $\begin{array}{l}\text { Age range: } 25 \text { to } 40 \text { years } \\
\text { Range of education levels: primary } \\
\text { school to bachelors degree } \\
\text { Majority lived in extended families } \\
\text { and they had one to three children. }\end{array}$ & $\begin{array}{l}\text { From abstract: "Five categories of breastfeeding attitudes and practices were identified: breast-feeding best but not } \\
\text { exclusive, cultural and traditional beliefs, infant feeding as a learning process, factors influencing decision to breastfeed, } \\
\text { and intention to feed the child.". } \\
\text { Additional notes: Mothers recognized that breastmilk is best, but none were exclusively breastfeeding. Many gave water } \\
\text { because they think the baby gets thirsty and that water will prevent tongue disease. Most discarded colostrum thinking it } \\
\text { is harmful for baby, but a few knew that it was beneficial to baby's health. Some were afraid breastmilk alone is not } \\
\text { enough for baby so they also gave formula. } \\
\text { Mothers believed that breastmilk quality was linked to health of mother (an ill mother would produce inferior milk), and } \\
\text { her ability to consume the traditional postnatal diet. Mothers followed traditional beliefs (e.g., what to eat and not eat; } \\
\text { messsaging) to improve breastmilk production. } \\
\text { Mothers, mothers-in-law, and grandmothers encouraged breastfeeding, told them how to breastfeed and helped with } \\
\text { housework to allow mother to rest (after childbirth) and care for infant. The process of learning about breastfeeding from } \\
\text { various sources gave them increased knowledge, which lead to improved self-confidence. Husband support gave } \\
\text { confidence in breastfeeding. Returning to work shortly after birth (mostly before } 6 \text { months) made breastfeeding difficult. } \\
\text { Media influenced mother's opinions of formula and baby foods and made them think they are good for baby. Lactation } \\
\text { problems (sore and cracked nipples, engorgement, inadequate lactation) interrupted breastfeeding with formula as a } \\
\text { replacement. Size of baby influenced breastfeeding: mothers said they would feed baby more if he/she was small. Some } \\
\text { mothers fed baby when he/she cried. After } 4 \text { months, mothers believed breastmilk alone was not enough so would add } \\
\text { formula and/or complementary foods. }\end{array}$ & $\begin{array}{l}(7) \\
(10) \\
(11) \\
(18) \\
(22) \\
(26) \\
(27) \\
(32) \\
(36) \\
(59) \\
(65) \\
(66) \\
(68)\end{array}$ \\
\hline
\end{tabular}




\begin{tabular}{|c|c|c|c|c|}
\hline $\begin{array}{c}\text { Study } \\
\text { No. }\end{array}$ & Author and Title & Participant Characteristics & Key Outcomes Reported & $\begin{array}{l}\text { Emergent Findings } \\
\text { (Finding Numbers } \\
\text { from Table 2) }\end{array}$ \\
\hline 8 & $\begin{array}{l}\text { Yati Afiyanti, Dyah Juliastuti } \\
\text { Exclusive breastfeeding practice in } \\
\text { Indonesia }\end{array}$ & Mothers were aged 19 to 36 years & $\begin{array}{l}\text { The initial breastfeeding practice immediately after delivery impeded the mothers' decisions to exclusively breastfeed. } \\
\text { For example, some women believed their baby had been given formula by the health provided after birth, so they } \\
\text { hesitated to breastfeed exclusively. } \\
\text { The belief of insufficient production of breast milk lead mothers to wean their babies early. } \\
\text { After weaning their babies, the mothers still had a desirir to breastfeed. Mothers kept trying to breastfeed even though } \\
\text { they had weaned their baby and reported breastmilk production had decline. Early weaning caused several } \\
\text { consequences (e.g., decline in breastmilk, increase in spending, health problems for the baby), which continued to affect } \\
\text { the mother's and baby's lives. Some women though their babies grew faster after weaning and they enjoyed the extra } \\
\text { time they had to spend on their daily activities. } \\
\text { Media promotion of formula, suggestions from peers and family, and the mothers need to work were reported as external } \\
\text { factors which impeded exclusive breastfeeding. Internal factors which did this included lack of knowledge about } \\
\text { exclusive breastfeeding, techniques to ensure effective breastfeeding (e.g., ensuring baby was satiated), not recognizing } \\
\text { baby feeding cues and behaviors, and believing formula can make babies healthier. }\end{array}$ & $\begin{array}{l}(5) \\
(8) \\
(9) \\
(10) \\
(22) \\
(26) \\
(28) \\
(32) \\
(33) \\
(34)\end{array}$ \\
\hline 9 & $\begin{array}{l}\text { Hope Mei Hong Lee, Jo Durham, } \\
\text { Jenny Booth, Vanphanom Sychareun } \\
\text { A qualitative study on the } \\
\text { breastfeeding experiences of } \\
\text { first-time mothers in Vientiane, } \\
\text { Lao PDR }\end{array}$ & $\begin{array}{l}\text { Mothers' ages ranged from } 19 \text { to } 39 . \\
\text { Three had attained primary } \\
\text { education; } 5 \text { had completed lower } \\
\text { secondary education; } 5 \text { had attained; } \\
\text { and } 3 \text { had finished tertiary education. } \\
\text { The majority of them were } \\
\text { unemployed and/or housewife ( } n= \\
\text { 9). Six were employed. }\end{array}$ & 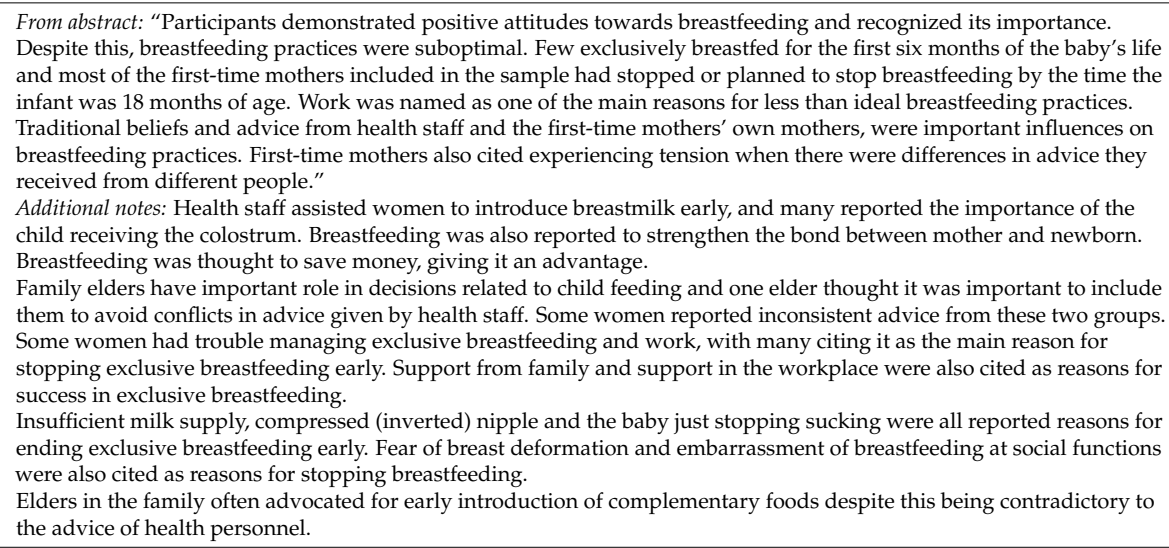 & $\begin{array}{c}(8) \\
(10) \\
(11) \\
(22) \\
(24) \\
(25) \\
(28) \\
(29) \\
(33) \\
(36) \\
(37) \\
(59) \\
(61) \\
(63) \\
(65) \\
(68)\end{array}$ \\
\hline 10 & $\begin{array}{l}\text { Christy Bomer-Norton } \\
\text { Timing of breastfeeding initiation in } \\
\text { rural Haiti: A focused ethnography }\end{array}$ & $\begin{array}{l}\text { The range of mothers' ages was } \\
\text { between } 19 \text { years and } 43 \text { years. Years } \\
\text { of education attainment ranged from } \\
0 \text { to } 13 \text { years. }\end{array}$ & $\begin{array}{l}\text { From abstract: "Mothers described baby care by birth attendants as well as actual and perceived health issues of the } \\
\text { mother and child as some of the factors influencing timing of breastfeeding initiation."” } \\
\text { Additional notes: All } 25 \text { mothers believed that breastfeeding, colostrum and mature milk, was good for baby's health and } \\
\text { they value providing these health benefits to their baby; baby's health was a priority for mothers. Mothers exhibited } \\
\text { comfort with breastfeeding (comfortable feeding in front of others) and had the feeling that breastfeeding was the normal } \\
\text { way of infant feeding. }\end{array}$ & $\begin{array}{c}(7) \\
(25) \\
(30) \\
(31) \\
(33) \\
(38) \\
(62) \\
(64) \\
(65)\end{array}$ \\
\hline
\end{tabular}




\begin{tabular}{|c|c|c|c|c|}
\hline $\begin{array}{l}\text { Study } \\
\text { No. }\end{array}$ & Author and Title & Participant Characteristics & Key Outcomes Reported & $\begin{array}{l}\text { Emergent Findings } \\
\text { (Finding Numbers } \\
\text { from Table 2) }\end{array}$ \\
\hline 11 & $\begin{array}{l}\text { Anne Laterra, Mohamed A. Ayoya, } \\
\text { Jean-Max Beauliere, } \\
\text { M'mbakwa Bienfait, Helena Pachon } \\
\text { Infant and young child feeding in } \\
\text { four departments in Haiti; } \\
\text { mixed-method study on prevalence } \\
\text { of recommended practices and } \\
\text { related attitudes, beliefs, and } \\
\text { other determinants }\end{array}$ & $\begin{array}{l}\text { Note: this was a mixed method study } \\
\text { with participants from the qualitative } \\
\text { portion being drawn from the } \\
\text { quantitative sample whose } \\
\text { charactersitics were described as: } \\
\text { "The majority of participants ( } 72.5 \%) \\
\text { were } 18-31 \text { years old. The mean age } \\
\text { of the most recent child was } 11.4 \\
\text { months and the mean number of } \\
\text { births was } 2.5 \text {. Maternal educational } \\
\text { status included (1) no formal } \\
\text { education (18.4\%); (2) completion of } \\
\text { primary school }(39.7 \%) \text {; and (3) } \\
\text { completion of secondary school } \\
\text { (37.1\%). The most recent birth of most } \\
\text { participants was attended by either a } \\
\text { physician (39.4\%) or a nurse }(19.7 \%) \text {. } \\
\text { The most recent births occurred in a } \\
\text { public hospital ( }(46.1 \%) \text { or in a home } \\
\text { (38.7\%)." }\end{array}$ & $\begin{array}{l}\text { Note: this was a mixed methods study. Only the qualitative results pertaining to this review are given here. } \\
\text { From abstract: "Qualitative data revealed that dietary diversity may be low because (1) mothers often struggle to } \\
\text { introduce complementary foods and (2) those that are traditionally introduced are not varied and primarily consist of } \\
\text { grains and starches.". } \\
\text { Additional notes: Qualitative data revealed several beliefs that may limit implementation of optimal IYCF practices. } \\
\text { Women in some communities felt it was necessary to administer purgatives shortly after birth to cleanse the child's body. } \\
\text { A poor diet is believed to weaken breast milk, making it inadequate in terms of quality and/or unsatisfying to the child. } \\
\text { Illness is thought to make breast milk "dirty" and unsafe for the child. In addition to these beliefs about breast milk, } \\
\text { women view continued breastfeeding as a possible risk for infections among older children and thus prefer earlier } \\
\text { weaning. Minimum dietary diversity achievement appears limited by difficulties mothers experience when } \\
\text { complementary feedings and the low diversity of traditional, grain-based complementary feeding. }\end{array}$ & $\begin{array}{l}(2) \\
(3) \\
(4) \\
(12) \\
(19) \\
(39) \\
(51) \\
(65)\end{array}$ \\
\hline 12 & $\begin{array}{l}\text { Gretel H. Pelto, } \\
\text { Margaret Armar-Klemesu } \\
\text { Identifying interventions to help } \\
\text { rural Kenyan mothers cope with food } \\
\text { insecurity: results of a focused } \\
\text { ethnographic study }\end{array}$ & Not stated & $\begin{array}{l}\text { From abstract: "The results provide qualitative evidence about facilitators and constraints to IYC nutrition in the two } \\
\text { geographical areas and document their inter-connections. We conclude with suggestions to consider } 13 \text { potential } \\
\text { nutrition-sensitive interventions. The studies provide empirical ethnographic support for arguments concerning the } \\
\text { importance of combining nutrition-specific and nutrition-sensitive interventions through a multi-sectoral, integrated } \\
\text { approach to improve the nutrition of infants and young children in low-income, resource-constrained populations.". } \\
\text { Additional notes: Not having enough money to buy food was commonly reported as a challenge associated with caring for } \\
\text { their children. Shortage of water /lack of access to clean water was also a difficulty, both in taking time from mothers in } \\
\text { water retrieval and in limiting food preparation. Collecting firewood and food storage limitations were also reported as } \\
\text { issues impacting food preparation for young children. Mothers knew the importance of food and nutrition to their child's } \\
\text { health and development. }\end{array}$ & $\begin{array}{l}(37) \\
(39) \\
(40) \\
(41) \\
(42) \\
(43) \\
(44)\end{array}$ \\
\hline
\end{tabular}




\begin{tabular}{|c|c|c|c|c|}
\hline $\begin{array}{c}\text { Study } \\
\text { No. }\end{array}$ & Author and Title & Participant Characteristics & Key Outcomes Reported & $\begin{array}{l}\text { Emergent Findings } \\
\text { (Finding Numbers } \\
\text { from Table 2) }\end{array}$ \\
\hline \multirow{2}{*}{\multicolumn{5}{|c|}{ Grey Literature }} \\
\hline \multirow{17}{*}{13} & \multirow{17}{*}{$\begin{array}{l}\text { Alessandra N Bazzano, } \\
\text { Richard A Oberhelman, } \\
\text { Kaitlin Storck Potts, Leah D Taub, } \\
\text { Chivorn Var } \\
\text { What health service support do } \\
\text { families need for optimal } \\
\text { breastfeeding? An in-depth } \\
\text { exploration of young infant feeding } \\
\text { practices in Cambodia }\end{array}$} & & & \\
\hline & & \multirow{16}{*}{$\begin{array}{l}\text { Mothers' ages ranged from } 21 \text { to } 55 . \\
\text { The range of education attainment } \\
\text { was between } 0 \text { and } 16 \text { years. Ten } \\
\text { mothers were housewives. Other } \\
\text { mothers worked as a factory worker } \\
(n=4) \text {, farmer }(n=3) \text {, or, } \\
\text { sewer /weaver }(n=4) \text {, The rest of } \\
\text { mothers' occupation included small } \\
\text { convenience store worker, clothes } \\
\text { seller, nighttime guard (father), and } \\
\text { high school teacher. }\end{array}$} & $\begin{array}{l}\text { barriers, particularly in the areas of infant latch, milk production, feeding frequency, and the use of breast milk } \\
\text { substitutes. The predominant theme identified in the research was a dearth of detailed information, advice, and }\end{array}$ & \\
\hline & & & $\begin{array}{l}\text { substitutes. The predominant theme identified in the research was a dearth of detailed information, advice, and } \\
\text { counseling for mothers beyond the message to exclusively breastfeed for } 6 \text { months." }\end{array}$ & (10) \\
\hline & & & Additional notes: Women knew to practice exclusive breastfeeding for 6 months and reported following this behavior, & (11) \\
\hline & & & however some mothers that reported this also said they used breastmilk substitutes on occasion. Some women described & (1) \\
\hline & & & giving prelacteal feeds or waiting for the milk to come in prior to initiating breastfeeding. Some mothers gave water to & \\
\hline & & & newborns. & (22) \\
\hline & & & Women reported that their own mothers, other female relatives or their midwives advised them on how to breastfeed. & (25) \\
\hline & & & One mother reported that the midwife advised her to exclusively breastfeed but did not give her specific instructions to & (26) \\
\hline & & & $\begin{array}{l}\text { do so. } \\
\text {. }\end{array}$ & $(28)$ \\
\hline & & & Participants believed milk supply was related to the mother's nutrition. Some felt they did not have enough milk and & (29) \\
\hline & & & one was concerned about transferring illness to her newborn via breastmilk. Some felt that newborns required less milk & (33) \\
\hline & & & $\begin{array}{l}\text { or shorter feedings (or from just one breast) than older babies required. Some also did not want to rouse a sleepy } \\
\text { newborn to feed. }\end{array}$ & (36) \\
\hline & & & Many women had concerns that breastfeeding would impact their breast shape and size. They especially felt this could & (59) \\
\hline & & & occur if they used both breasts during one feeding session. & (62) \\
\hline & & & Positioning the newborn was also difficult for some mothers. & \\
\hline & & & $\begin{array}{l}\text { Observations confirmed problems of poor latch, pain from suboptimal infant positioning and the practice of feeding from } \\
\text { only one breast per session. }\end{array}$ & \\
\hline \multirow{17}{*}{14} & \multirow{17}{*}{$\begin{array}{l}\text { Jennifer Burns, Jillian A. Emerson, } \\
\text { Kimberly Amundson, } \\
\text { Shannon Doocy, Laura E. Caulfield, } \\
\text { Rolf D. W. Klemm } \\
\text { A Qualitative Analysis of Barriers } \\
\text { and Facilitators to Optimal } \\
\text { Breastfeeding and Complementary } \\
\text { Feeding Practices in South Kivu, } \\
\text { Democratic Republic of Congo }\end{array}$} & \multirow{17}{*}{$\begin{array}{l}\text { The mean age of mothers was } \\
25.8 \text { years. } \\
\text { Almost half of the mothers were } \\
\text { literate }(44 \%) \text {, however, around } 28 \% \\
\text { had never attended school. } \\
\text { Most of the mothers reported small } \\
\text { businesses }(47 \%) \text { and agriculture } \\
(46 \%) \text { as sources of income for } \\
\text { their households. }\end{array}$} & From abstract: "Although breastfeeding was prevalent, few mothers engaged in optimal feeding practices. Barriers & \\
\hline & & & & \\
\hline & & & Health provider guidance and mothers' motivation to breastfeed and feed nutrient-dense foods emerged as facilitators to & (11) \\
\hline & & & & \\
\hline & & & Additional notes: Many women recognized the benefits of colostrum for their newborn and gave it to them. Many said & (19) \\
\hline & & & their mothers or grandmothers did not practice this and discarded the colostrum, but advice from health centers has & (22) \\
\hline & & & changed this behavior. Common reasons for discontinuing exclusive breastfeeding included the feeling of insufficient & (31) \\
\hline & & & milk, clogged milk duct, and the child being ill. These reason led to early introduction of complementary foods, water, or & (32) \\
\hline & & & sugar water. The weather being hot was another reason cited for & \\
\hline & & & was considered an indication of the child being unsatisfied & \\
\hline & & & complementary foods like porridges. They were encouraged to continu this practice when giving other foods led to the & (37) \\
\hline & & & baby stopping crying. & (39) \\
\hline & & & Women who succeeded in exclusive breastfeeding for 6 months had encouragement during ANC visits. Mothers also & (41) \\
\hline & & & reported that their own diets were important to the success of exclusive breastfeeding. & (45) \\
\hline & & & Children's diets (older than 6 months) have little diversity, and they eat what the family eats. Families with livestock do & 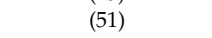 \\
\hline & & & not tend to give the meat and milk available to their children as they prefer to sell these products in the market. The little & (63) \\
\hline & & & meat the family eats is usually purchased, and milk is not commonly given to children as it is expensive. & \\
\hline
\end{tabular}




\begin{tabular}{|c|c|c|c|c|}
\hline $\begin{array}{l}\text { Study } \\
\text { No. }\end{array}$ & Author and Title & Participant Characteristics & Key Outcomes Reported & $\begin{array}{l}\text { Emergent Findings } \\
\text { (Finding Numbers } \\
\text { from Table 2) }\end{array}$ \\
\hline \multicolumn{5}{|c|}{ Grey Literature } \\
\hline 15 & $\begin{array}{l}\text { Alive and Thrive-Ethiopia } \\
\text { IYCF Practices, Beliefs, and } \\
\text { Influences in Tigrey Region, Ethiopia }\end{array}$ & Not stated & $\begin{array}{l}\text { From summary: "The findings from this study showed that although mothers have basic breastfeeding and } \\
\text { complementary feeding information, visible gaps remain, such as not practicing exclusive breastfeeding, the existence of } \\
\text { faulty traditional beliefs, and specific misconceptions about breastfeeding and complementary feeding. } \\
\text { Among the major misconceptions is the belief of mothers that giving only breastmilk without adding fenugreek juice will } \\
\text { expose the baby to intestinal worms. Beliefs also keep mothers from providing extra breastfeeding for a sick child. Some } \\
\text { mothers reported the belief that encouraging suckling when the child is ill will only contribute to its illness. } \\
\text { In Tigrey Region, mothers, community members, and HEWs alike stressed the unavailability of diverse foods and lack of } \\
\text { resources to purchase foods as the major reasons for not following recommended complementary feeding practices. Lack } \\
\text { of food was also mentioned in explaining why mothers introduce complementary foods later than } 6 \text { months of age, fail to } \\
\text { offer children a variety of food types, and are unable to give children extra meals when they are sick or recovering from } \\
\text { illness. } \\
\text { The study identified misconceptions that affect complementary feeding habits. Mothers, community leaders, and even } \\
\text { voluntary community health promoters (VCHPs) hold widespread beliefs that children cannot digest meat or other } \\
\text { animal products, that children will choke on thick porridges, that extra food when a child is sick will contribute to illness, } \\
\text { that children will refuse to eat during or after sickness, and that bottle feeding is more sanitary than using cups or hands } \\
\text { to feed. } \\
\text { Mothers and other community members, HEWs, VCHPs, and supervisors all expressed interest in learning about the } \\
\text { recommended IYCF practices, as long as they trust that the person providing advice is properly trained and has reliable } \\
\text { information. } \\
\text { The findings from this study reveal that HEWs have reasonably better knowledge in the area of breastfeeding than in } \\
\text { complementary feeding. Regarding their own knowledge and skills related to counseling, almost all of the HEWs are } \\
\text { keen to improve those skills." }\end{array}$ & $\begin{array}{l}(8) \\
(20) \\
(22) \\
(33) \\
(37) \\
(39) \\
(40) \\
(41) \\
(42) \\
(48) \\
(49) \\
(50) \\
(51) \\
(53) \\
(54) \\
(55) \\
(56) \\
(57) \\
(63) \\
(65) \\
(71) \\
(72) \\
(73)\end{array}$ \\
\hline 16 & $\begin{array}{l}\text { Alive and Thrive } \\
\text { IYCF Practices, Beliefs, and } \\
\text { Influences in SNNP Region, Ethiopia }\end{array}$ & Not stated & $\begin{array}{l}\text { From summary: This study indicates that breastfeeding is widely practiced in the research communities and is expected of } \\
\text { all mothers and valued in the community for its contribution towards child growth and healthy development. } \\
\text { Mothers in the study areas seem to know the expected breastfeeding requirements such as: on-demand feeding, } \\
\text { frequency of breastfeeeding, and the need to feed from both breasts. Yet exclusive breastfeeding is not widely practiced in } \\
\text { the study communities. Mothers commonly introduce water, fenugreek, or linseed water as early as } 2 \text { months. } \\
\text { In regards to complementary foods, the study indicates that the age for introduction of additional foods ranges from } 2 \\
\text { months to } 8 \text { months, with a majority of mothers reporting that they had started giving foods at } 6 \text { months or earlier. } \\
\text { Certain foods such as kale, enichila, banana, egg, pumpkin, carrot, and green vegetables are considered unsuitable and } \\
\text { "too strong" for young children to digest before } 1 \text { year of age. Mothers consider meat in particular as too difficult to } \\
\text { digest. Most mothers recommend meat for children above } 1 \text { or } 2 \text { years old. Mothers also expressed that they need to have } \\
\text { a good income to buy or feed their children such foods. } \\
\text { The study also revealed that HEWs feel they have a need to learn more about child feeding in order to effectively counsel } \\
\text { mothers on IYCF. Several pointed out that they need more detailed information about additional foods, types of foods, } \\
\text { and best approaches for preparing food for young children, with follow-up mechanism. }\end{array}$ & $\begin{array}{l}(5) \\
(8) \\
(10) \\
(11) \\
(18) \\
(22) \\
(27) \\
(39) \\
(41) \\
(51) \\
(52) \\
(53) \\
(55) \\
(56) \\
(62) \\
(63) \\
(65) \\
(70) \\
(71) \\
(72)\end{array}$ \\
\hline
\end{tabular}




\begin{tabular}{|c|c|c|c|c|}
\hline $\begin{array}{c}\text { Study } \\
\text { No. }\end{array}$ & Author and Title & Participant Characteristics & Key Outcomes Reported & $\begin{array}{l}\text { Emergent Findings } \\
\text { (Finding Numbers } \\
\text { from Table 2) }\end{array}$ \\
\hline 17 & $\begin{array}{l}\text { United States Agency for } \\
\text { International Development } \\
\text { Formative assessment of infant and } \\
\text { young child feeding practices at the } \\
\text { community level in Zambia }\end{array}$ & Not stated & $\begin{array}{l}\text { Breastfeeding is recognized as extremely important by mothers and other members of the community and remains the } \\
\text { first choice for infant feeding. There is widespread awareness about the message of exclusive breastfeeding for six } \\
\text { months, and generally mothers, fathers and grandmothers expressed their support for the practice. However, in reality } \\
\text { many respondents expressed doubts as to whether breastmilk was really sufficient during the first six months and } \\
\text { whether mothers could produce enough breastmilk to meet their child's needs. In particular, families tended to introduce } \\
\text { water before six months due to their perception that infants gesture to indicate their needs - and the belief that not } \\
\text { responding is cruel to the child. And it is traditional to introduce water and watery foods early. When women are away } \\
\text { from their infants, most respondents did not feel it was acceptable to express breastmill. Many respondents strongly } \\
\text { oppose it because it is considered untraditional, unhygienic, and unnecessary if the mother can stay close to her child. } \\
\text { Multiple respondents commented that when mothers have an inadequate diet, they do not produce enough breastmilk, } \\
\text { and need to introduce foods and liquids before } 6 \text { monthts of age, or must wean their child from breastmilk. } \\
\text { The study identified a number of factors that represent barriers to the uptake of recommended infant and young child } \\
\text { feeding practices. Respondents noted that cultural beliefs, traditions, and lack of knowledge about the nutritional needs } \\
\text { of young children are barriers (e.g., most women give thin unenriched porridges as complementary foods). Many } \\
\text { respondents sidentified lack of food and inability to afford food as a major reason for not providing appropriate foods to } \\
\text { young children. } \\
\text { Mothers did not cite grandmothers and fathers as important influencers of IYCF. The media was cited as a source of } \\
\text { information on IYCF. }\end{array}$ & $\begin{array}{l}(8) \\
(10) \\
(11) \\
(18) \\
(19) \\
(20) \\
(22) \\
(31) \\
(32) \\
(33) \\
(34) \\
(37) \\
(39) \\
(41) \\
(62) \\
(63) \\
(65)\end{array}$ \\
\hline 18 & $\begin{array}{l}\text { United States Agency for } \\
\text { International Development } \\
\text { Formative assessment of infant and } \\
\text { young child feeding practices-federal } \\
\text { capital territory, Nigeria }\end{array}$ & Not stated & $\begin{array}{l}\text { Prelacteal feeding of water and sometimes water with herbs was widespread. There were different beliefs regarding } \\
\text { colostrum but some believed (with influence by the grandmother) that it was bad and dangerous for the infant. A } \\
\text { traditional practice is to give herbal infusions in water to treat and prevent illness, from the time of birth. Being away } \\
\text { from baby inhibited breastfeeding, and often results in introduction of water. Many of the women are farmers and it is } \\
\text { difficult to breastfeed when they are working on the farm. Breastmilk is not believed to be sufficient in either water or } \\
\text { sustenance for babies } 3 \text { months and older, so thin gruels are introduced at this age. Some women believed that a mother } \\
\text { with HIV could not breastfeed her baby. } \\
\text { A sick child with a lack of appetite requires "forced feeding" but some felt concern that this might make the child choke } \\
\text { (child sickness impacts child feeding). } \\
\text { Maternal nutrition is recognized as important for pregnant and lactating women, and inadequate nutrition leads to } \\
\text { inadequate milk production, but there was a lack of consensus of what foods are best for mothers and affordability may } \\
\text { impact their abilitity to attain good foods. Fathers were supportive of the women and children achieving good nutrition by } \\
\text { letting them eat first. }\end{array}$ & $\begin{array}{l}(7) \\
(17) \\
(18) \\
(19) \\
(20) \\
(22) \\
(27) \\
(32) \\
(33) \\
(37) \\
(47) \\
(50) \\
(62) \\
(63) \\
(65) \\
(70) \\
(72) \\
(73)\end{array}$ \\
\hline
\end{tabular}




\begin{tabular}{|c|c|c|c|c|}
\hline $\begin{array}{c}\text { Study } \\
\text { No. }\end{array}$ & Author and Title & Participant Characteristics & Key Outcomes Reported & $\begin{array}{l}\text { Emergent Findings } \\
\text { (Finding Numbers } \\
\text { from Table 2) }\end{array}$ \\
\hline 19 & $\begin{array}{l}\text { United States Agency for } \\
\text { International Development } \\
\text { Consulting with caregivers-formative } \\
\text { research to determine the barriers } \\
\text { and facilitators to optimal infant and } \\
\text { young child feeding in three regions } \\
\text { of Malawi }\end{array}$ & $\begin{array}{l}\text { Mothers in both phases were young, } \\
\text { with more than half in each sample } \\
\text { less than } 25 \text { years old. The overall } \\
\text { literacy rate of the women } \\
\text { in both samples (63.3 percent in } \\
\text { Phase } 1 \text { and } 69 \text { percent in Phase } 2) \\
\text { was slightly less than the national } \\
\text { literacy average of } 70 \text { percent, } 19 \text { and } \\
\text { in both samples, there was a wide } \\
\text { variation in years of schooling, with } \\
\text { approximately one-fifth to } \\
\text { one-quarter of each group having no } \\
\text { schooling at all and another quarter } \\
\text { having more than six years } \\
\text { of schooling. }\end{array}$ & $\begin{array}{l}\text { Crying after feeding influenced the mother or other family members to give the baby other foods or liquids, assuming } \\
\text { that the breastmilk was inadequate to satiate the baby's hunger. Giving water during hot times of the year was thought to } \\
\text { be necessary by some mothers, interfering with exclusive breastfeeding. Mothers did not know that breastmilk alone was } \\
\text { acceptable for infants less than } 6 \text { months; once they were told this and tried it many were successful at transitioning to } \\
\text { the improved practice. } \\
\text { Observations identified two suboptimal breastfeeding practices: breastfeeds of very short duration, and breastfeeding } \\
\text { from just one breast. When mothers transitions to improved practices, positive outcomes reinforced this behavior, } \\
\text { including less crying and better sleep for babies, which give mothers more time for household chores, babies took more } \\
\text { milk and did not have an upset stomach. Other family members support also facilitated good practice, for example by } \\
\text { identifying that longer duration of breastfeeding indicated mother was showing more love to the child. } \\
\text { Fear of HIV transmission was indicated as a reason for stopping continued breastfeeding early, along with having } \\
\text { another pregnancy. The study also found that using breastfeeding to prevent pregnancy was also a facilitator of } \\
\text { continued breastfeeding, but that mothers need counseling on the use of this method. } \\
\text { Families given infants } 6 \text { to } 8 \text { months thin/watery porridges because they think this is the only food this age child can } \\
\text { swallow and digest. }\end{array}$ & $\begin{array}{c}(8) \\
(10) \\
(11) \\
(18) \\
(21) \\
(31) \\
(34) \\
(38) \\
(41) \\
(51) \\
(53) \\
(55) \\
(63) \\
(65) \\
(66) \\
(73)\end{array}$ \\
\hline 20 & $\begin{array}{l}\text { Academy for Educational } \\
\text { Development (AED)/ } \\
\text { Alive and Thrive } \\
\text { Ethiopia Country Office } \\
\text { Initial Insight Mining and Pretest } \\
\text { Research for Alive and Thrive } \\
\text { Ethiopia }\end{array}$ & Not stated & $\begin{array}{l}\text { Breastfeeding is common, but exclusive breastfeeding remains difficult for most mothers are they are responsible for a lot } \\
\text { of work both in and out of the home. They are very busy and have limited access to information, do not have time to } \\
\text { prepare special foods for children or exclusively breastfeed for six months (especially for mothers who work in the fields). } \\
\text { Overall, there is a lack of awareness about nutritional requirements for infants and young children. Adding oil to baby } \\
\text { food is common, and meat is considered healthy but not given to children under } 2 \text { years because it is thought babies } \\
\text { cannot digest meat. Thick porridge is also considered to be hard to digest, so thin porridge is given to young children. } \\
\text { Eggs are sold in the market rather than given to children because they are expensive; the same applies to milk- if they } \\
\text { have a cow they sell the milk in market, and many cannot afford a cow or goat. There was a lack of awareness that } \\
\text { different foods provided different nutrients, or that nutrition was related to mental and physical development. } \\
\text { Respondents were not aware of the importance of the first two years of life. } \\
\text { In most locations, there is lack of availability of several food categories, especially meat and fish, and they are expensive } \\
\text { when they are available. }\end{array}$ & $\begin{array}{l}(14) \\
(21) \\
(22) \\
(32) \\
(33) \\
(39) \\
(41) \\
(45) \\
(51) \\
(55)\end{array}$ \\
\hline
\end{tabular}




\begin{tabular}{|c|c|c|c|c|}
\hline $\begin{array}{c}\text { Study } \\
\text { No. }\end{array}$ & Author and Title & Participant Characteristics & Key Outcomes Reported & $\begin{array}{l}\text { Emergent Findings } \\
\text { (Finding Numbers } \\
\text { from Table 2) }\end{array}$ \\
\hline 21 & $\begin{array}{l}\text { United States Agency for } \\
\text { International Development } \\
\text { Engaging grandmothers and men in } \\
\text { infant and young child feeing and } \\
\text { maternal nutrition—-Report of a } \\
\text { formative assessment in Eastern and } \\
\text { Western Kenya }\end{array}$ & Not stated & 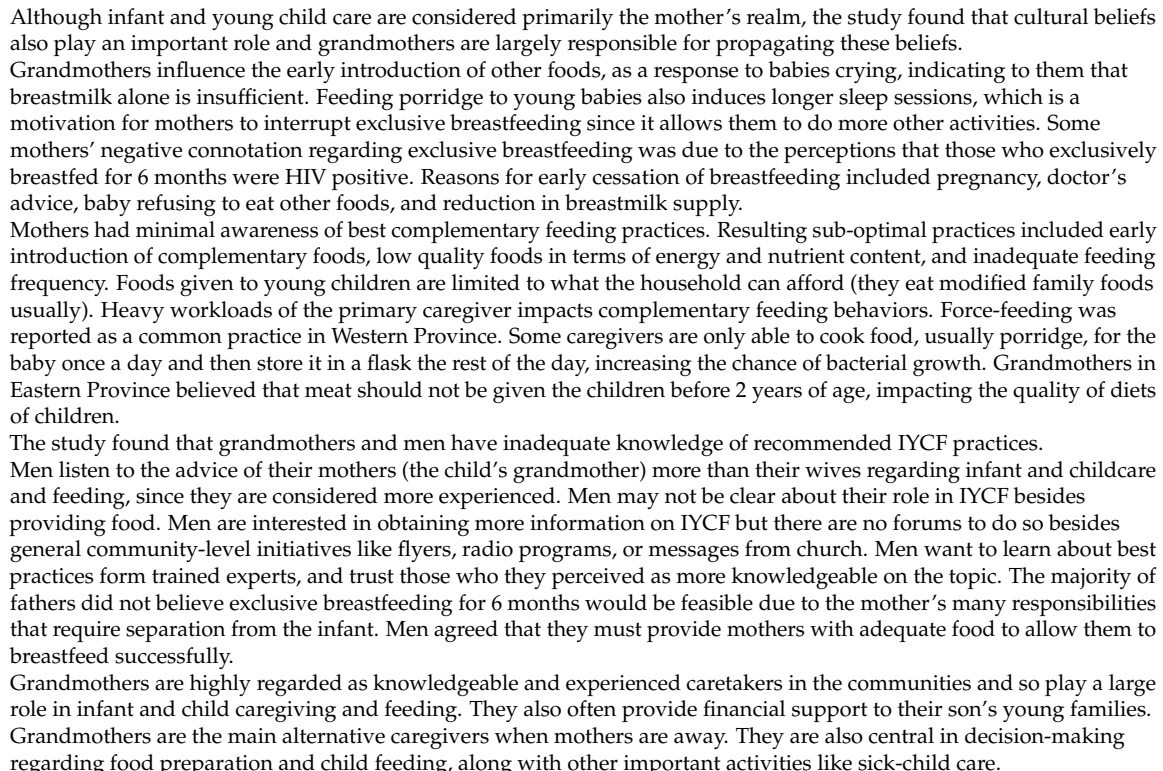 & $\begin{array}{c}(8) \\
(10) \\
(11) \\
(16) \\
(18) \\
(19) \\
(20) \\
(21) \\
(27) \\
(33) \\
(37) \\
(39) \\
(51) \\
(58) \\
(65) \\
(71) \\
(73)\end{array}$ \\
\hline
\end{tabular}




\begin{tabular}{|c|c|c|c|c|}
\hline $\begin{array}{c}\text { Study } \\
\text { No. }\end{array}$ & Author and Title & Participant Characteristics & Key Outcomes Reported & $\begin{array}{l}\text { Emergent Findings } \\
\text { (Finding Numbers } \\
\text { from Table 2) }\end{array}$ \\
\hline \multicolumn{5}{|c|}{ Grey Literature } \\
\hline 22 & $\begin{array}{l}\text { United States Agency for } \\
\text { International Development } \\
\text { Ghana promotion of complementary } \\
\text { feeding practices project-baseline } \\
\text { survey report }\end{array}$ & $\begin{array}{l}\text { The majority of respondents }(42.2 \%) \\
\text { were between the ages of } 22 \text { and } 29 \\
\text { years. The median age was } 28 \text { years. } \\
\text { More than half of the respondents } \\
(56.3 \%) \text { in the nine districts had little } \\
\text { or no education (primary school or } \\
\text { less). Only one-third had completed } \\
\text { basic education (middle school or } \\
\text { junior high school). District-specific } \\
\text { data showed a similar result in only } \\
\text { three of the districts: Sene, Sunyani } \\
\text { West, and Kintampo South having } \\
\text { respondents with tertiary level } \\
\text { education. The majority were peasant } \\
\text { farmers, including a few fishermen } \\
(55.2 \%) \text {; followed by petty traders } \\
(13.7 \%) \text { and artisans (12.2\%). A little } \\
\text { more than } 10 \%(11.5 \%) \text { reported that } \\
\text { they were unemployed. About } 85 \% \\
\text { of the respondents were married. } \\
\text { Only } 7 \% \text { of participants were single } \\
\text { (never married), and } 6.3 \% \text { were } \\
\text { cohabiting. The majority of the study } \\
\text { participants reported Christianity as } \\
\text { their religion ( } 81.5 \%) \text {. This was } \\
\text { followed by Islam (11.5\%), with a } \\
\text { small percentage being traditionalists. }\end{array}$ & $\begin{array}{l}\text { Note: The majority of findings presented are from the quantitative portions of this study, so are not relevant for this review. } \\
\text { Mothers and fathers who believed exclusive breastfeeding was good for baby }<6 \text { months said that it helps a baby grow } \\
\text { and protects them from breastfeeding. } \\
\text { Those who did not believe exclusive breastfeeding was best said that this was because a baby needs more than just } \\
\text { breastmilk to satisfy them (they also need water). }\end{array}$ & $\begin{array}{l}(18) \\
(33) \\
(65) \\
(70)\end{array}$ \\
\hline 23 & $\begin{array}{l}\text { Alive and Thrive Bangladesh } \\
\text { Perceptions, practices, and promotion } \\
\text { of infant and young child } \\
\text { feeding-results and program } \\
\text { implications of assessments } \\
\text { in Bangladesh }\end{array}$ & Not stated & $\begin{array}{l}\text { From summary: "Delayed initiation of breastfeeding, pre-lacteal feeding, and lack of exclusive breastfeeding were found } \\
\text { to be common largely due to a lack of understanding of their importance and confusion about colostrum feeding as well } \\
\text { as the definition of EBF. Also, mothers lack support for appropriate infant feeding after delivery. The perception that } \\
\text { baby's crying indicates insufficient treastmilk and that insufficient breastmilk is caused by poor maternal diets is } \\
\text { widespread and a major barrier to EBF.". } \\
\text { Additional notes: Perceptions of what foods should be given for complementary feeding of children } 6 \text { to } 24 \text { months differ } \\
\text { from the recommended practices. There is a lack of diversity of type of food available to give children, especially animal } \\
\text { sourced foods. Child's poor appetite causes them to reject foods, inhibiting optimal feeding. Mother's time constraints } \\
\text { and lack of knowledge of appropriate complementary feeding were viewed as larger barriers than limited food } \\
\text { availability. Mothers lack knowledge of how to make family foods appropriate for young babies and therefore do not } \\
\text { give them meat because it is presumed to be not appropriate for them. }\end{array}$ & $\begin{array}{l}(10) \\
(11) \\
(19) \\
(24) \\
(39) \\
(46) \\
(48) \\
(51) \\
(55)\end{array}$ \\
\hline
\end{tabular}




\section{Appendix C}

Details of CERQual qualitative evidence profile of barriers and facilitators to recommended breastfeeding and complementary feeding practices.

\begin{tabular}{|c|c|c|c|c|c|c|c|c|}
\hline $\begin{array}{l}\text { Finding } \\
\text { No. }\end{array}$ & Review Finding & $\begin{array}{c}\text { Studies } \\
\text { Contributing to the } \\
\text { Review Finding }\end{array}$ & $\begin{array}{l}\text { Assessment of } \\
\text { Methodological } \\
\text { Limitations }\end{array}$ & $\begin{array}{l}\text { Assessment of } \\
\text { Relevance }\end{array}$ & Assessment of Coherence & Assessment of Adequacy & $\begin{array}{l}\text { Overall CERQual } \\
\text { Assessment of } \\
\text { Confidence }\end{array}$ & Explanation of Judgement \\
\hline \multirow{2}{*}{\multicolumn{9}{|c|}{$\begin{array}{c}\text { Barriers } \\
\text { Barriers to Recommended Breastfeeding }\end{array}$}} \\
\hline \multicolumn{5}{|c|}{$\begin{array}{c}\text { Barriers to Recommended Breastfeeding } \\
\text { Key Theme: Beliefs and Perceptions }\end{array}$} & & & & \\
\hline 1 & $\begin{array}{l}\text { Breastfeeding } \\
\text { deforms breasts }\end{array}$ & $1 ; 4 ; 13$ & $\begin{array}{l}\text { Minor methodological } \\
\text { limitations (the authors } \\
\text { do not clarify } \\
\text { potential bias) }\end{array}$ & $\begin{array}{l}\text { Minor concerns about } \\
\text { relevance (the finding } \\
\text { comes from studies in } \\
\text { three settings across } \\
\text { different locales: } \\
\text { Cambodia, Dominican } \\
\text { Republic, and Ghana) }\end{array}$ & $\begin{array}{l}\text { Minor concerns about } \\
\text { coherence (data consistent } \\
\text { within and across both } \\
\text { studies) }\end{array}$ & $\begin{array}{l}\text { No concern about adequacy } \\
\text { of data (the authors provide } \\
\text { rich data) }\end{array}$ & High confidence & $\begin{array}{l}\text { This finding was graded as high } \\
\text { confidence because of few } \\
\text { concerns about coherence and } \\
\text { relevance and moderate } \\
\text { methodological limitations. }\end{array}$ \\
\hline 2 & $\begin{array}{l}\text { Breastfeeding for } \\
\text { more than one year } \\
\text { makes children } \\
\text { susceptible } \\
\text { to infection }\end{array}$ & 11 & $\begin{array}{l}\text { Minor methodological } \\
\text { limitations (the authors } \\
\text { do not state } \\
\text { potential bias) }\end{array}$ & $\mathrm{NA}$ & NA & $\begin{array}{l}\text { Minor concern about } \\
\text { adequacy (the authors offer } \\
\text { moderately rich data overall) }\end{array}$ & $\mathrm{NA}$ & $\mathrm{NA}$ \\
\hline 3 & $\begin{array}{l}\text { Breastmilk is bad } \\
\text { because a mother } \\
\text { is mad }\end{array}$ & 11 & $\begin{array}{l}\text { Minor methodological } \\
\text { limitations (the authors } \\
\text { do not state } \\
\text { potential bias) }\end{array}$ & $\mathrm{NA}$ & NA & $\begin{array}{l}\text { Minor concern about } \\
\text { adequacy (the authors offer } \\
\text { moderately rich data overall) }\end{array}$ & $\mathrm{NA}$ & $\mathrm{NA}$ \\
\hline 4 & $\begin{array}{l}\text { Breastmilk is dirty } \\
\text { because a mother has } \\
\text { an illness }\end{array}$ & 11 & $\begin{array}{l}\text { Minor methodological } \\
\text { limitations (the authors } \\
\text { do not state } \\
\text { potential bias) }\end{array}$ & $\mathrm{NA}$ & NA & $\begin{array}{l}\text { Minor concern about } \\
\text { adequacy (the authors offer } \\
\text { moderately rich data overall) }\end{array}$ & $\mathrm{NA}$ & $\mathrm{NA}$ \\
\hline 5 & $\begin{array}{l}\text { Breastmilk is not } \\
\text { enough for a } \\
\text { small baby }\end{array}$ & $8 ; 16$ & $\begin{array}{l}\text { Substantial } \\
\text { methodological } \\
\text { limitations (both } \\
\text { studies do not explain } \\
\text { the sampling method, } \\
\text { eligibility criteria and } \\
\text { potential bias) }\end{array}$ & $\begin{array}{l}\text { Moderate concerns about } \\
\text { relevance (partial } \\
\text { relevance, as the studies } \\
\text { were from only two } \\
\text { settings: Indonesia } \\
\text { and Ethiopia) }\end{array}$ & $\begin{array}{l}\text { Minor concerns about } \\
\text { coherence (data consistent } \\
\text { within and across both } \\
\text { studies) }\end{array}$ & $\begin{array}{l}\text { Moderate concerns about } \\
\text { adequacy (only two studies, } \\
\text { one provides rich data, but } \\
\text { the other one offers thin data) }\end{array}$ & Low confidence & $\begin{array}{l}\text { This finding was graded as low } \\
\text { confidence because of moderate } \\
\text { concerns about relevance and } \\
\text { adequacy of data and substantial } \\
\text { methodological limitations. }\end{array}$ \\
\hline 6 & $\begin{array}{l}\text { When baby belches } \\
\text { on the breast during } \\
\text { breastfeeding the } \\
\text { mother can develop } \\
\text { swollen breasts }\end{array}$ & 4 & $\begin{array}{l}\text { Moderate } \\
\text { methodological } \\
\text { limitations (the study } \\
\text { does not state analysis } \\
\text { methods and } \\
\text { potential bias) }\end{array}$ & $\mathrm{NA}$ & NA & $\begin{array}{l}\text { No concern about adequacy } \\
\text { of data (the authors provide } \\
\text { rich data) }\end{array}$ & $\mathrm{NA}$ & $\mathrm{NA}$ \\
\hline 7 & $\begin{array}{l}\text { Colostrum is not } \\
\text { healthy for baby, so } \\
\text { mothers discard } \\
\text { colostrum }\end{array}$ & $3 ; 7 ; 10 ; 18$ & $\begin{array}{l}\text { Moderate } \\
\text { methodological } \\
\text { limitations (all studies } \\
\text { do not discuss potential } \\
\text { bias; two studies do not } \\
\text { state analysis methods; } \\
\text { one does not say } \\
\text { eligibility criteria) }\end{array}$ & $\begin{array}{l}\text { Minor concerns about } \\
\text { relevance (studies of } \\
\text { breastfeeding from four } \\
\text { countries: Ghana, } \\
\text { Vietnam, Haiti and } \\
\text { Nigeria) }\end{array}$ & $\begin{array}{l}\text { Minor concerns about } \\
\text { coherence (data reasonably } \\
\text { consistent within and across } \\
\text { all studies) }\end{array}$ & $\begin{array}{l}\text { Minor concerns about } \\
\text { adequacy (three studies offer } \\
\text { rich data overall while one } \\
\text { study shows thin data) }\end{array}$ & Moderate confidence & $\begin{array}{l}\text { This finding was graded as } \\
\text { moderate confidence because of } \\
\text { minor concerns regarding } \\
\text { relevance, coherence, and } \\
\text { adequacy and moderate } \\
\text { methodological limitations. }\end{array}$ \\
\hline
\end{tabular}




\begin{tabular}{|c|c|c|c|c|c|c|c|c|}
\hline $\begin{array}{l}\text { Finding } \\
\text { No. }\end{array}$ & Review Finding & $\begin{array}{c}\text { Studies } \\
\text { Contributing to the } \\
\text { Review Finding }\end{array}$ & $\begin{array}{c}\text { Assessment of } \\
\text { Methodological } \\
\text { Limitations }\end{array}$ & $\begin{array}{l}\text { Assessment of } \\
\text { Relevance }\end{array}$ & Assessment of Coherence & Assessment of Adequacy & $\begin{array}{l}\text { Overall CERQual } \\
\text { Assessment of } \\
\text { Confidence }\end{array}$ & Explanation of Judgement \\
\hline 8 & $\begin{array}{l}\text { Baby remains hungry } \\
\text { after breastfeeding } \\
\text { (e.g., a baby is fussy } \\
\text { even after } \\
\text { breastfeeding) }\end{array}$ & $\begin{array}{c}5 ; 6 ; 8 ; 9 ; 14 ; 15 ; 16 ; 17 \\
19 ; 21\end{array}$ & $\begin{array}{l}\text { Moderate } \\
\text { methodological } \\
\text { limitations (all studies } \\
\text { with moderate } \\
\text { methodological } \\
\text { limitations) }\end{array}$ & $\begin{array}{l}\text { Minor concerns about } \\
\text { relevance (the review } \\
\text { finding is applicable from } \\
\text { four countries: Colombia, } \\
\text { Ethiopia, Zambia } \\
\text { and Kenya) }\end{array}$ & $\begin{array}{l}\text { Minor concerns about } \\
\text { coherence (data reasonably } \\
\text { consistent within and across } \\
\text { all studies) }\end{array}$ & $\begin{array}{l}\text { Moderate concerns about } \\
\text { adequacy (two studies } \\
\text { provide rich data, but three } \\
\text { studies offer only thin data) }\end{array}$ & Moderate confidence & $\begin{array}{l}\text { This finding was graded as } \\
\text { moderate confidence because of } \\
\text { moderate concerns regarding } \\
\text { methodological limitations and } \\
\text { adequacy of data and minor } \\
\text { concerns about relevance } \\
\text { and coherence. }\end{array}$ \\
\hline 9 & $\begin{array}{l}\text { Formula milk can } \\
\text { make a baby healthier }\end{array}$ & 8 & $\begin{array}{l}\text { Moderate } \\
\text { methodological } \\
\text { limitations (the authors } \\
\text { do not explain the } \\
\text { sampling method, } \\
\text { eligibility criteria and } \\
\text { potential bias) }\end{array}$ & NA & NA & $\begin{array}{l}\text { No concern about adequacy } \\
\text { (the authors provide } \\
\text { rich data) }\end{array}$ & NA & NA \\
\hline 10 & $\begin{array}{l}\text { Mothers do not } \\
\text { produce sufficient } \\
\text { breastmilk }\end{array}$ & $\begin{array}{c}3 ; 4 ; 5 ; 6 ; 7 ; 8 ; 9 ; 13 ; 14 \\
\quad 16 ; 17 ; 1 ; ; 21 ; 23\end{array}$ & $\begin{array}{l}\text { Moderate } \\
\text { methodological } \\
\text { limitations (all studies } \\
\text { with moderate } \\
\text { methodological } \\
\text { limitations) }\end{array}$ & $\begin{array}{l}\text { Minor concerns about } \\
\text { relevance (the review } \\
\text { finding is universal in } 13 \\
\text { countries: Cambodia, } \\
\text { Democratic Republic of } \\
\text { Congo, Ghana, Colombia, } \\
\text { Bangladesh, Vietnam, } \\
\text { Indonesia, Laos PDR, } \\
\text { Ethiopia, Zambia, } \\
\text { Malawi, Kenya and } \\
\text { Bangladesh) }\end{array}$ & $\begin{array}{l}\text { Minor concerns about } \\
\text { coherence (data reasonably } \\
\text { consistent within and across } \\
\text { all studies) }\end{array}$ & $\begin{array}{l}\text { Minor concerns about } \\
\text { adequacy (the majority of the } \\
\text { studies provide rich data. } \\
\text { Only one study offers } \\
\text { thin data) }\end{array}$ & High confidence & $\begin{array}{l}\text { This finding was graded as high } \\
\text { confidence because of minor } \\
\text { concerns about relevance, } \\
\text { coherence and adequacy and } \\
\text { moderate } \\
\text { methodological limitations. }\end{array}$ \\
\hline 11 & A baby is thirsty & $\begin{array}{c}2 ; 7 ; 9 ; 13 ; 14 ; 16 ; 17 \\
19 ; 21 ; 23\end{array}$ & $\begin{array}{l}\text { Moderate } \\
\text { methodological } \\
\text { limitations (all studies } \\
\text { with moderate } \\
\text { methodological } \\
\text { limitations) }\end{array}$ & $\begin{array}{l}\text { Minor concerns about } \\
\text { relevance (the review } \\
\text { finding is relevant across } \\
\text { nine countries: } \\
\text { Cambodia, Democratic } \\
\text { Republic of Congo, } \\
\text { Tanzania, Vietnam, Lao } \\
\text { PDR, Ethiopia, Zambia, } \\
\text { Malawi, Kenya and } \\
\text { Bangladesh) }\end{array}$ & $\begin{array}{l}\text { Minor concerns about } \\
\text { coherence (data reasonably } \\
\text { consistent within and across } \\
\text { all studies) }\end{array}$ & $\begin{array}{l}\text { Moderate concerns about } \\
\text { adequacy (six studies } \\
\text { provide moderately rich data } \\
\text { overall, but four studies offer } \\
\text { thin data) }\end{array}$ & Moderate confidence & $\begin{array}{l}\text { This finding was graded as } \\
\text { moderate confidence because of } \\
\text { moderate concerns regarding } \\
\text { methodological limitations and } \\
\text { adequacy of data and minor } \\
\text { concerns about relevance } \\
\text { and coherence. }\end{array}$ \\
\hline 12 & $\begin{array}{l}\text { Purgative cleans } \\
\text { a baby }\end{array}$ & 11 & $\begin{array}{l}\text { Minor methodological } \\
\text { limitations (the authors } \\
\text { do not state } \\
\text { potential bias) }\end{array}$ & NA & NA & $\begin{array}{l}\text { Substantial concerns about } \\
\text { adequacy (only short quotes } \\
\text { are available) }\end{array}$ & NA & NA \\
\hline 13 & $\begin{array}{l}\text { Arrival of breastmilk } \\
\text { is late }\end{array}$ & 3 & $\begin{array}{l}\text { Moderate } \\
\text { methodological } \\
\text { limitations (the authors } \\
\text { do not explain analysis } \\
\text { methods and } \\
\text { potential bias) }\end{array}$ & NA & NA & $\begin{array}{l}\text { No concern about adequacy } \\
\text { of data (the authors provide } \\
\text { rich data) }\end{array}$ & NA & NA \\
\hline
\end{tabular}




\begin{tabular}{|c|c|c|c|c|c|c|c|c|}
\hline $\begin{array}{l}\text { Finding } \\
\text { No. }\end{array}$ & Review Finding & $\begin{array}{c}\text { Studies } \\
\text { Contributing to the } \\
\text { Review Finding }\end{array}$ & $\begin{array}{c}\text { Assessment of } \\
\text { Methodological } \\
\text { Limitations }\end{array}$ & $\begin{array}{l}\text { Assessment of } \\
\text { Relevance }\end{array}$ & Assessment of Coherence & Assessment of Adequacy & $\begin{array}{l}\text { Overall CERQual } \\
\text { Assessment of } \\
\text { Confidence }\end{array}$ & Explanation of Judgement \\
\hline 14 & $\begin{array}{l}\text { Giving fenugreek and } \\
\text { traditional herbs to } \\
\text { newborns or young } \\
\text { babies is a } \\
\text { traditional practice }\end{array}$ & 20 & $\begin{array}{l}\text { Moderate } \\
\text { methodological } \\
\text { limitations (the authors } \\
\text { do not state sampling, } \\
\text { analysis method and } \\
\text { potential bias. }\end{array}$ & NA & $\mathrm{NA}$ & $\begin{array}{l}\text { Minor concerns about } \\
\text { adequacy (the authors offer } \\
\text { only thin data) }\end{array}$ & $\mathrm{NA}$ & NA \\
\hline 15 & $\begin{array}{l}\text { Mothers do not have } \\
\text { confidence in } \\
\text { sufficient production } \\
\text { of breast milk }\end{array}$ & 5 & $\begin{array}{l}\text { Moderate } \\
\text { methodological } \\
\text { limitations (the authors } \\
\text { do not state sampling } \\
\text { and potential bias) }\end{array}$ & $\mathrm{NA}$ & NA & $\begin{array}{l}\text { No concern about adequacy } \\
\text { of data (the study provides } \\
\text { rich data) }\end{array}$ & $\mathrm{NA}$ & NA \\
\hline 16 & $\begin{array}{l}\text { A baby still cries even } \\
\text { after a mother } \\
\text { breastfeeds }\end{array}$ & 21 & $\begin{array}{l}\text { Moderate } \\
\text { methodological } \\
\text { limitations (the authors } \\
\text { do not state sampling } \\
\text { and potential bias) }\end{array}$ & NA & NA & $\begin{array}{l}\text { No concern about adequacy } \\
\text { of data (the authors provide } \\
\text { rich data) }\end{array}$ & NA & NA \\
\hline 17 & $\begin{array}{l}\text { Herbal infusions in } \\
\text { water prevents a baby } \\
\text { from diseases. }\end{array}$ & 18 & $\begin{array}{l}\text { Moderate } \\
\text { methodological } \\
\text { limitations (the authors } \\
\text { do not state sampling } \\
\text { and potential bias) }\end{array}$ & NA & $\mathrm{NA}$ & $\begin{array}{l}\text { No concern about adequacy } \\
\text { of data (the study shows } \\
\text { rich data) }\end{array}$ & NA & NA \\
\hline 18 & $\begin{array}{l}\text { Traditional practices } \\
\text { do not require } \\
\text { exclusive } \\
\text { breastfeeding (e.g., } \\
\text { pre-lacteal feeds, } \\
\text { giving water) }\end{array}$ & $\begin{array}{c}2 ; 7 ; 14 ; 16 ; 17 ; 18 ; 19 ; \\
21 ; 22\end{array}$ & $\begin{array}{l}\text { Moderate } \\
\text { methodological } \\
\text { limitations (all studies } \\
\text { with moderate } \\
\text { methodological } \\
\text { limitations) }\end{array}$ & $\begin{array}{l}\text { Minor concerns about } \\
\text { relevance (the review } \\
\text { finding is relevant across } \\
\text { nine countries: } \\
\text { Democratic Republic of } \\
\text { Congo, Tanzania, } \\
\text { Vietnam, Ethiopia, } \\
\text { Zambia, Nigeria, Malawi, } \\
\text { Kenya and Ghana) }\end{array}$ & $\begin{array}{l}\text { Moderate concerns about } \\
\text { coherence (data in four } \\
\text { studies lack a convincing } \\
\text { explanation for the patterns } \\
\text { found in these data while } \\
\text { data in other five studies } \\
\text { relatively are consistent } \\
\text { within and across studies) }\end{array}$ & $\begin{array}{l}\text { Moderate concerns about } \\
\text { adequacy of data (three } \\
\text { studies with thin data. Six } \\
\text { studies with rich data). }\end{array}$ & Moderate confidence & $\begin{array}{l}\text { This finding was graded as } \\
\text { moderate confidence because of } \\
\text { minor concerns about relevance } \\
\text { and moderate concerns regarding } \\
\text { methodological limitations and } \\
\text { coherence and adequacy of data. }\end{array}$ \\
\hline 19 & $\begin{array}{l}\text { Mothers attribute } \\
\text { their inability to } \\
\text { produce enough } \\
\text { breastmilk to not } \\
\text { eating well }\end{array}$ & $\begin{array}{c}11 ; 13 ; 14 ; 17 ; 18 ; 21 ; \\
23\end{array}$ & $\begin{array}{l}\text { Moderate } \\
\text { methodological } \\
\text { limitations (five studies } \\
\text { with moderate } \\
\text { methodological } \\
\text { limitations and one } \\
\text { study with minor } \\
\text { methodological } \\
\text { limitations) }\end{array}$ & $\begin{array}{l}\text { Minor concerns about } \\
\text { relevance (the review } \\
\text { finding is universal in } \\
\text { seven countries: } \\
\text { Democratic Republic of } \\
\text { Congo, Zambia, Nigeria, } \\
\text { Kenya, Haiti, Cambodia } \\
\text { and Bangladesh) }\end{array}$ & $\begin{array}{l}\text { Minor concerns about } \\
\text { coherence (data reasonably } \\
\text { consistent within and across } \\
\text { all studies) }\end{array}$ & $\begin{array}{l}\text { Minor concerns about } \\
\text { adequacy of data (all data } \\
\text { provides moderately rich } \\
\text { data overall) }\end{array}$ & High confidence & $\begin{array}{l}\text { This finding was graded as high } \\
\text { confidence because of minor } \\
\text { concerns about relevance, } \\
\text { coherence and adequacy and } \\
\text { moderate } \\
\text { methodological limitations. }\end{array}$ \\
\hline 20 & $\begin{array}{l}\text { Pregnant women } \\
\text { should not breastfeed } \\
\text { (Mothers become } \\
\text { pregnant before the } \\
\text { child is } 2 \text { years old) }\end{array}$ & $15 ; 17 ; 18 ; 21$ & $\begin{array}{l}\text { Moderate } \\
\text { methodological } \\
\text { limitations (all studies } \\
\text { with moderate } \\
\text { methodological } \\
\text { limitations) }\end{array}$ & $\begin{array}{l}\text { Minor concerns about } \\
\text { relevance (the review } \\
\text { finding is relevant across } \\
\text { four countries: Ethiopia, } \\
\text { Zambia, Nigeria and } \\
\text { Kenya) }\end{array}$ & $\begin{array}{l}\text { Minor concerns about } \\
\text { coherence (data reasonably } \\
\text { consistent within and across } \\
\text { all studies) }\end{array}$ & $\begin{array}{l}\text { Moderate concerns about } \\
\text { adequacy of data (two } \\
\text { studies offer relatively rich } \\
\text { data, but other studies show } \\
\text { only thin data) }\end{array}$ & Moderate confidence & $\begin{array}{l}\text { This finding was graded as } \\
\text { moderate confidence because of } \\
\text { minor concerns about relevance, } \\
\text { coherence and moderate concerns } \\
\text { about methodological limitations } \\
\text { and adequacy of data. }\end{array}$ \\
\hline
\end{tabular}




\begin{tabular}{|c|c|c|c|c|c|c|c|c|}
\hline $\begin{array}{l}\text { Finding } \\
\text { No. }\end{array}$ & Review Finding & $\begin{array}{c}\text { Studies } \\
\text { Contributing to the } \\
\text { Review Finding }\end{array}$ & $\begin{array}{c}\text { Assessment of } \\
\text { Methodological } \\
\text { Limitations }\end{array}$ & $\begin{array}{l}\text { Assessment of } \\
\text { Relevance }\end{array}$ & Assessment of Coherence & Assessment of Adequacy & $\begin{array}{l}\text { Overall CERQual } \\
\text { Assessment of } \\
\text { Confidence }\end{array}$ & Explanation of Judgement \\
\hline \multicolumn{9}{|c|}{ Key Theme: Lack of Support from Families, Health Workers (HWs) and Others } \\
\hline 21 & $\begin{array}{l}\text { Family members do } \\
\text { not support mothers } \\
\text { to breastfeed }\end{array}$ & $4 ; 19 ; 20 ; 21$ & $\begin{array}{l}\text { Moderate } \\
\text { methodological } \\
\text { limitations (all studies } \\
\text { with moderate } \\
\text { methodological } \\
\text { limitations) }\end{array}$ & $\begin{array}{l}\text { Minor concerns about } \\
\text { relevance (the review } \\
\text { finding is relevant across } \\
\text { four countries: Ghana, } \\
\text { Kenya, Malawi and } \\
\text { Ethiopia) }\end{array}$ & $\begin{array}{l}\text { Minor concerns about } \\
\text { coherence (data reasonably } \\
\text { consistent within and across } \\
\text { all studies) }\end{array}$ & $\begin{array}{l}\text { Substantial concerns about } \\
\text { adequacy (three studies } \\
\text { provide poor data. Only one } \\
\text { study offers rich data). }\end{array}$ & Moderate confidence & $\begin{array}{l}\text { This finding was graded as } \\
\text { moderate confidence because of } \\
\text { minor concerns about coherence } \\
\text { and relevance; moderate } \\
\text { methodological limitations; and } \\
\text { substantial concerns about } \\
\text { adequacy. }\end{array}$ \\
\hline 22 & $\begin{array}{l}\text { Mothers do not have } \\
\text { enough time to } \\
\text { breastfeed their } \\
\text { infants due to their } \\
\text { work and short } \\
\text { maternity leave }\end{array}$ & $\begin{array}{c}4 ; 6 ; 7 ; 8 ; 9 ; 13 ; 14 ; 15 ; \\
16 ; 17 ; 18 ; 20\end{array}$ & $\begin{array}{l}\text { Moderate } \\
\text { methodological } \\
\text { limitations (all studies } \\
\text { with moderate } \\
\text { methodological } \\
\text { limitations) }\end{array}$ & $\begin{array}{l}\text { Minor concerns about } \\
\text { relevance (the review } \\
\text { finding is relevant across } \\
\text { ten countries: Ghana, } \\
\text { Vietnam, Bangladesh, } \\
\text { Indonesia, Lao PDR, } \\
\text { Cambodia, Democratic } \\
\text { Republic of Congo, } \\
\text { Ethiopia, Zambia } \\
\text { and Nigeria) }\end{array}$ & $\begin{array}{l}\text { Minor concerns about } \\
\text { coherence (data reasonably } \\
\text { consistent within and across } \\
\text { all studies) }\end{array}$ & $\begin{array}{l}\text { Moderate concerns about } \\
\text { adequacy (the majority of the } \\
\text { studies provide moderately } \\
\text { rich data overall, but three } \\
\text { studies offer thin data) }\end{array}$ & Moderate confidence & $\begin{array}{l}\text { This finding was graded as } \\
\text { moderate confidence because of } \\
\text { minor concerns about relevance } \\
\text { and moderate concerns regarding } \\
\text { methodological limitations and } \\
\text { coherence and adequacy of data. }\end{array}$ \\
\hline 23 & $\begin{array}{l}\text { HWs believe mothers } \\
\text { do not produce } \\
\text { enough breastmilk }\end{array}$ & 3 & $\begin{array}{l}\text { Moderate } \\
\text { methodological } \\
\text { limitations (the authors } \\
\text { do not explain analysis } \\
\text { methods and potential } \\
\text { bias) }\end{array}$ & NA & NA & $\begin{array}{l}\text { No concern about adequacy } \\
\text { of data (the authors provide } \\
\text { rich data) }\end{array}$ & NA & NA \\
\hline 24 & $\begin{array}{l}\text { HWs do not have } \\
\text { adequate knowledge } \\
\text { and skills to help } \\
\text { mothers }\end{array}$ & $2 ; 9 ; 23$ & $\begin{array}{l}\text { Moderate } \\
\text { methodological } \\
\text { limitations (all studies } \\
\text { have moderate } \\
\text { methodological } \\
\text { limitations) }\end{array}$ & $\begin{array}{l}\text { Moderate concerns about } \\
\text { relevance (partial } \\
\text { relevance, as the studies } \\
\text { were from only two } \\
\text { settings: Tanzania, Laos } \\
\text { and Bangladesh) }\end{array}$ & $\begin{array}{l}\text { Minor concerns about } \\
\text { coherence (data consistent } \\
\text { within and across both } \\
\text { studies) }\end{array}$ & $\begin{array}{l}\text { Minor concerns about } \\
\text { adequacy (all studies provide } \\
\text { rich data) }\end{array}$ & Moderate confidence & $\begin{array}{l}\text { This finding was graded as } \\
\text { moderate confidence because of } \\
\text { minor concerns about coherence } \\
\text { and adequacy; moderate concern } \\
\text { about relevance and } \\
\text { methodological limitations. }\end{array}$ \\
\hline 25 & $\begin{array}{l}\text { Mothers do not } \\
\text { receive any advice } \\
\text { from HWs }\end{array}$ & $3 ; 9 ; 10 ; 13$ & $\begin{array}{l}\text { Minor methodological } \\
\text { limitations (two studies } \\
\text { with minor limitations } \\
\text { and the other studies } \\
\text { with moderate } \\
\text { limitations) }\end{array}$ & $\begin{array}{l}\text { Minor concerns about } \\
\text { relevance (the review } \\
\text { finding is universal } \\
\text { across four countries: } \\
\text { Ghana, Laos PDR, Haiti } \\
\text { and Cambodia) }\end{array}$ & $\begin{array}{l}\text { Minor concerns about } \\
\text { coherence (data consistent } \\
\text { within and across both } \\
\text { studies) }\end{array}$ & $\begin{array}{l}\text { Minor concerns about } \\
\text { adequacy (three studies } \\
\text { provide rich data while only } \\
\text { one study offers thin data) }\end{array}$ & High confidence & $\begin{array}{l}\text { This finding was graded as high } \\
\text { confidence because of few } \\
\text { concerns about methodological } \\
\text { limitations, relevance, coherence } \\
\text { and adequacy. }\end{array}$ \\
\hline 26 & $\begin{array}{l}\text { Media has an impact } \\
\text { on use of formula } \\
\text { milk (and encourages } \\
\text { it by instilling the } \\
\text { belief that formula is } \\
\text { healthy for baby) }\end{array}$ & $7 ; 8 ; 13$ & $\begin{array}{l}\text { Moderate } \\
\text { methodological } \\
\text { limitations (all studies } \\
\text { do not consider } \\
\text { potential bias. One } \\
\text { study do not describe } \\
\text { sampling methods) }\end{array}$ & $\begin{array}{l}\text { Minor concerns about } \\
\text { relevance (the review } \\
\text { finding is relevant across } \\
\text { three countries in Asia: } \\
\text { Indonesia, Cambodia and } \\
\text { Vietnam) }\end{array}$ & $\begin{array}{l}\text { Minor concerns about } \\
\text { coherence (data consistent } \\
\text { within and across both } \\
\text { studies) }\end{array}$ & $\begin{array}{l}\text { No concern about adequacy } \\
\text { of data (both studies offer } \\
\text { rich data) }\end{array}$ & High confidence & $\begin{array}{l}\text { This finding was graded as high } \\
\text { confidence because of few } \\
\text { concerns about coherence and } \\
\text { relevance and moderate } \\
\text { methodological limitations. }\end{array}$ \\
\hline
\end{tabular}




\begin{tabular}{|c|c|c|c|c|c|c|c|c|}
\hline $\begin{array}{l}\text { Finding } \\
\text { No. }\end{array}$ & Review Finding & $\begin{array}{c}\text { Studies } \\
\text { Contributing to the } \\
\text { Review Finding }\end{array}$ & $\begin{array}{l}\text { Assessment of } \\
\text { Methodological } \\
\text { Limitations }\end{array}$ & $\begin{array}{l}\text { Assessment of } \\
\text { Relevance }\end{array}$ & Assessment of Coherence & Assessment of Adequacy & $\begin{array}{l}\text { Overall CERQual } \\
\text { Assessment of } \\
\text { Confidence }\end{array}$ & Explanation of Judgement \\
\hline 27 & $\begin{array}{l}\text { Traditional cultural } \\
\text { beliefs of female } \\
\text { family members (e.g., } \\
\text { grandmothers) } \\
\text { influence } \\
\text { breastfeeding }\end{array}$ & $7 ; 16 ; 18 ; 21$ & $\begin{array}{l}\text { Moderate } \\
\text { methodological } \\
\text { limitations (all studies } \\
\text { with moderate } \\
\text { methodological } \\
\text { limitations) }\end{array}$ & $\begin{array}{l}\text { Minor concerns about } \\
\text { relevance (the review } \\
\text { finding is relevant across } \\
\text { four countries: Vietnam, } \\
\text { Ethiopia, Kenya } \\
\text { and Nigeria) }\end{array}$ & $\begin{array}{l}\text { Minor concerns about } \\
\text { coherence (data consistent } \\
\text { within and across } \\
\text { both studies) }\end{array}$ & $\begin{array}{l}\text { Moderate concerns about } \\
\text { adequacy (two studies show } \\
\text { poor data. The other studies } \\
\text { provide moderately rich } \\
\text { data overall) }\end{array}$ & Moderate confidence & $\begin{array}{l}\text { This finding was graded as } \\
\text { moderate confidence because of } \\
\text { minor concerns about coherence } \\
\text { and relevance; moderate concern } \\
\text { about methodological limitations } \\
\text { and adequacy. }\end{array}$ \\
\hline 28 & $\begin{array}{l}\text { Suggestions from } \\
\text { families, peers or } \\
\text { close neighbors } \\
\text { influence } \\
\text { breastfeeding }\end{array}$ & $8 ; 9 ; 13$ & $\begin{array}{l}\text { Moderate } \\
\text { methodological } \\
\text { limitations (two studies } \\
\text { do not consider } \\
\text { potential bias. One } \\
\text { does not state sampling. } \\
\text { One does not explain } \\
\text { analysis method) }\end{array}$ & $\begin{array}{l}\text { Minor concerns about } \\
\text { relevance (partial } \\
\text { relevance, as the studies } \\
\text { were from three settings: } \\
\text { Cambodia, Indonesia } \\
\text { and Laos) }\end{array}$ & $\begin{array}{l}\text { Minor concerns about } \\
\text { coherence (data consistent } \\
\text { within and across } \\
\text { both studies) }\end{array}$ & $\begin{array}{l}\text { No concern about adequacy } \\
\text { of data (all studies offer } \\
\text { rich data) }\end{array}$ & High confidence & $\begin{array}{l}\text { This finding was graded as high } \\
\text { confidence because of adequate } \\
\text { data, minor concerns about } \\
\text { coherence and relevance and } \\
\text { moderate concerns about } \\
\text { methodological limitations }\end{array}$ \\
\hline \multicolumn{9}{|c|}{ Key Theme: Infant-Specific Factors } \\
\hline 29 & $\begin{array}{l}\text { A baby stops } \\
\text { sucking milk }\end{array}$ & $9 ; 13$ & $\begin{array}{l}\text { Moderate } \\
\text { methodological } \\
\text { limitations (one study } \\
\text { do not explain potential } \\
\text { bias. The other one } \\
\text { states response bias and } \\
\text { does not explain } \\
\text { analysis methods) }\end{array}$ & $\begin{array}{l}\text { Moderate concerns about } \\
\text { relevance (partial } \\
\text { relevance, as the studies } \\
\text { were from only two } \\
\text { settings: Cambodia } \\
\text { and Laos) }\end{array}$ & $\begin{array}{l}\text { Minor concerns about } \\
\text { coherence (data consistent } \\
\text { within and across } \\
\text { both studies) }\end{array}$ & $\begin{array}{l}\text { No concern about adequacy } \\
\text { of data (both studies offer } \\
\text { rich data) }\end{array}$ & Moderate confidence & $\begin{array}{l}\text { This finding was graded as } \\
\text { moderate confidence because of } \\
\text { minor concerns about coherence, } \\
\text { moderate concern about relevance } \\
\text { and methodological limitations }\end{array}$ \\
\hline 30 & $\begin{array}{l}\text { Delayed } \\
\text { breastfeeding } \\
\text { initiation occurs with } \\
\text { infants with } \\
\text { respiratory problems } \\
\end{array}$ & 10 & $\begin{array}{l}\text { Minor methodological } \\
\text { limitations (the study } \\
\text { has some missing data) }\end{array}$ & $\mathrm{NA}$ & NA & $\begin{array}{l}\text { No concern about adequacy } \\
\text { of data (rich data) }\end{array}$ & $\mathrm{NA}$ & $\mathrm{NA}$ \\
\hline 31 & Baby has a sickness & $4 ; 10 ; 14 ; 17 ; 19$ & $\begin{array}{l}\text { Moderate } \\
\text { methodological } \\
\text { limitations (the } \\
\text { majority of the studies } \\
\text { have moderate } \\
\text { methodological } \\
\text { limitations. Only one } \\
\text { study has minor } \\
\text { limitations. }\end{array}$ & $\begin{array}{l}\text { Minor concerns about } \\
\text { relevance (the review } \\
\text { finding is relevant across } \\
\text { five countries: Ghana, } \\
\text { Haiti, Democratic } \\
\text { Republic of Congo, } \\
\text { Nigeria and Malawi) }\end{array}$ & $\begin{array}{l}\text { Minor concerns about } \\
\text { coherence (data consistent } \\
\text { within and across both } \\
\text { studies) }\end{array}$ & $\begin{array}{l}\text { Substantial concerns about } \\
\text { adequacy (only one study } \\
\text { offers rich data. Others show } \\
\text { thin data) }\end{array}$ & Moderate confidence & $\begin{array}{l}\text { This finding was graded as } \\
\text { moderate confidence because of } \\
\text { substantial concerns about } \\
\text { adequacy; moderate } \\
\text { methodological limitations; and } \\
\text { minor concerns regarding } \\
\text { coherence and relevance. }\end{array}$ \\
\hline
\end{tabular}




\begin{tabular}{|c|c|c|c|c|c|c|c|c|}
\hline $\begin{array}{l}\text { Finding } \\
\text { No. }\end{array}$ & Review Finding & $\begin{array}{c}\text { Studies } \\
\text { Contributing to the } \\
\text { Review Finding }\end{array}$ & $\begin{array}{l}\text { Assessment of } \\
\text { Methodological } \\
\text { Limitations }\end{array}$ & $\begin{array}{l}\text { Assessment of } \\
\text { Relevance }\end{array}$ & Assessment of Coherence & Assessment of Adequacy & $\begin{array}{l}\text { Overall CERQual } \\
\text { Assessment of } \\
\text { Confidence }\end{array}$ & Explanation of Judgement \\
\hline \multicolumn{9}{|c|}{ Key Theme: Mothers' Knowledge } \\
\hline 32 & $\begin{array}{l}\text { Mothers do not have } \\
\text { knowledge about } \\
\text { colostrum and } \\
\text { breastfeeding } \\
\text { initiation }\end{array}$ & $3 ; 7 ; 8 ; 14 ; 17 ; 18 ; 20$ & $\begin{array}{l}\text { Moderate } \\
\text { methodological } \\
\text { limitations (only one } \\
\text { study with minor } \\
\text { methodological } \\
\text { limitations. Other } \\
\text { studies with moderate } \\
\text { methodological } \\
\text { limitations) }\end{array}$ & $\begin{array}{l}\text { Minor concerns about } \\
\text { relevance (the review } \\
\text { finding is relevant across } \\
\text { seven countries: Ghana, } \\
\text { Vietnam, Indonesia, } \\
\text { Democratic Republic of } \\
\text { Congo, Zambia, Nigeria } \\
\text { and Ethiopia) }\end{array}$ & $\begin{array}{l}\text { Minor concerns about } \\
\text { coherence (data consistent } \\
\text { within and across } \\
\text { both studies) }\end{array}$ & $\begin{array}{l}\text { Substantial concerns about } \\
\text { adequacy (only three studies } \\
\text { provide moderately rich data } \\
\text { overall. Others offer } \\
\text { thin data) }\end{array}$ & Moderate confidence & $\begin{array}{l}\text { This finding was graded as } \\
\text { moderate confidence because of } \\
\text { substantial concerns about } \\
\text { adequacy; moderate } \\
\text { methodological limitations; and } \\
\text { minor concerns regarding } \\
\text { coherence and relevance }\end{array}$ \\
\hline 33 & $\begin{array}{l}\text { Mothers do not have } \\
\text { knowledge about } \\
\text { breastfeeding }\end{array}$ & $\begin{array}{c}8 ; 9 ; 10 ; 13 ; 14 ; 15 ; 17 \\
18 ; 20 ; 21 ; 22\end{array}$ & $\begin{array}{l}\text { Moderate } \\
\text { methodological } \\
\text { limitations (only one } \\
\text { study with minor } \\
\text { methodological } \\
\text { limitations. Other } \\
\text { studies with moderate } \\
\text { methodological } \\
\text { limitations) }\end{array}$ & $\begin{array}{l}\text { Minor concerns about } \\
\text { relevance (the review } \\
\text { finding is relevant across } \\
\text { ten countries: Indonesia, } \\
\text { Lao PDR, Haiti, } \\
\text { Cambodia, Democratic } \\
\text { Republic of Congo, } \\
\text { Zambia, Nigeria, } \\
\text { Ethiopia, Kenya } \\
\text { and Ghana) }\end{array}$ & $\begin{array}{l}\text { Moderate concerns about } \\
\text { coherence (three studies lack } \\
\text { a convincing explanation. } \\
\text { Data in other studies } \\
\text { consistent within and across } \\
\text { all studies) }\end{array}$ & $\begin{array}{l}\text { Moderate concerns about } \\
\text { adequacy (three studies } \\
\text { provide poor data. Other } \\
\text { studies offer moderately rich } \\
\text { data overall) }\end{array}$ & Moderate confidence & $\begin{array}{l}\text { This finding was graded as } \\
\text { moderate confidence because of } \\
\text { moderate concerns regarding } \\
\text { methodology, coherence, adequacy } \\
\text { and minor concerns about } \\
\text { relevance. }\end{array}$ \\
\hline 34 & $\begin{array}{l}\text { Mothers do not } \\
\text { recognize between } \\
\text { baby feeding cues } \\
\text { and behaviors }\end{array}$ & $8 ; 17 ; 19$ & $\begin{array}{l}\text { Moderate } \\
\text { methodological } \\
\text { limitations (all studies } \\
\text { with moderate } \\
\text { methodological } \\
\text { limitations) }\end{array}$ & $\begin{array}{l}\text { Moderate concerns about } \\
\text { relevance (the review } \\
\text { finding is found only in } \\
\text { three settings: Indonesia, } \\
\text { Zambia and Malawi) }\end{array}$ & $\begin{array}{l}\text { Minor concerns about } \\
\text { coherence (data consistent } \\
\text { within and across both } \\
\text { studies) }\end{array}$ & $\begin{array}{l}\text { Minor concerns about } \\
\text { adequacy (all studies provide } \\
\text { moderately rich data) }\end{array}$ & Moderate confidence & $\begin{array}{l}\text { This finding was graded as } \\
\text { moderate confidence because of } \\
\text { moderate concerns about } \\
\text { methodology, relevance, coherence } \\
\text { and minor concerns about } \\
\text { adequacy. }\end{array}$ \\
\hline 35 & $\begin{array}{l}\text { Mothers do not have } \\
\text { education }\end{array}$ & 2 & $\begin{array}{l}\text { Moderate } \\
\text { methodological } \\
\text { limitations (the authors } \\
\text { do not explain } \\
\text { sampling and potential } \\
\text { bias) }\end{array}$ & NA & NA & $\begin{array}{l}\text { Minor concern about } \\
\text { adequacy of data (the } \\
\text { authors provide moderately } \\
\text { rich data overall) }\end{array}$ & NA & NA \\
\hline \multicolumn{9}{|c|}{ Key Theme: Mother-Specific Factors } \\
\hline 36 & $\begin{array}{l}\text { Mothers have breast } \\
\text { and nipple problems }\end{array}$ & $4 ; 5 ; 7 ; 9 ; 13 ; 14$ & $\begin{array}{l}\text { Moderate } \\
\text { methodological } \\
\text { limitations (all studies } \\
\text { with moderate } \\
\text { methodological } \\
\text { limitations) }\end{array}$ & $\begin{array}{l}\text { Minor concerns about } \\
\text { relevance (the review } \\
\text { finding is relevant across } \\
\text { seven countries: Ghana, } \\
\text { Colombia, Vietnam, Lao } \\
\text { PDR, Cambodia and } \\
\text { Democratic Republic } \\
\text { of Congo) }\end{array}$ & $\begin{array}{l}\text { Minor concerns about } \\
\text { coherence (data consistent } \\
\text { within and across } \\
\text { both studies) }\end{array}$ & $\begin{array}{l}\text { Minor concern about } \\
\text { adequacy of data (the } \\
\text { majority of the studies } \\
\text { provide moderately rich data } \\
\text { overall, but one study shows } \\
\text { only thin data) }\end{array}$ & High confidence & $\begin{array}{l}\text { This finding was graded as high } \\
\text { confidence because of minor } \\
\text { concerns regarding relevance, } \\
\text { coherence, adequacy and moderate } \\
\text { methodological limitations. }\end{array}$ \\
\hline
\end{tabular}




\begin{tabular}{|c|c|c|c|c|c|c|c|c|}
\hline $\begin{array}{l}\text { Finding } \\
\text { No. }\end{array}$ & Review Finding & $\begin{array}{c}\text { Studies } \\
\text { Contributing to the } \\
\text { Review Finding }\end{array}$ & $\begin{array}{c}\text { Assessment of } \\
\text { Methodological } \\
\text { Limitations }\end{array}$ & $\begin{array}{l}\text { Assessment of } \\
\text { Relevance }\end{array}$ & Assessment of Coherence & Assessment of Adequacy & $\begin{array}{l}\text { Overall CERQual } \\
\text { Assessment of } \\
\text { Confidence }\end{array}$ & Explanation of Judgement \\
\hline 37 & $\begin{array}{l}\text { Mothers have a } \\
\text { sickness and/or died }\end{array}$ & $\begin{array}{c}4 ; 9 ; 12 ; 13 ; 14 ; 15 ; 17 \\
18 ; 21\end{array}$ & $\begin{array}{l}\text { Moderate } \\
\text { methodological } \\
\text { limitations (the most } \\
\text { studies with moderate } \\
\text { methodological } \\
\text { limitations except one } \\
\text { study with } \\
\text { minor limitations) }\end{array}$ & $\begin{array}{l}\text { Minor concerns about } \\
\text { relevance (the review } \\
\text { finding is relevant across } \\
\text { seven countries: Ghana, } \\
\text { Lao PDR, Kenya, } \\
\text { Cambodia, Democratic } \\
\text { Republic of Congo, } \\
\text { Ethiopia, Zambia, } \\
\text { and Nigeria) }\end{array}$ & $\begin{array}{l}\text { Minor concerns about } \\
\text { coherence (data consistent } \\
\text { within and across } \\
\text { both studies) }\end{array}$ & $\begin{array}{l}\text { Substantial concerns about } \\
\text { adequacy (only three studies } \\
\text { show rich data, other studies } \\
\text { provide only thin data) }\end{array}$ & Moderate confidence & $\begin{array}{l}\text { This finding was graded as } \\
\text { moderate confidence because of } \\
\text { substantial concerns about } \\
\text { adequacy of data, moderate } \\
\text { methodological limitations and } \\
\text { minor concerns regarding } \\
\text { relevance and coherence. }\end{array}$ \\
\hline 38 & $\begin{array}{l}\text { Breastfeeding } \\
\text { initiation is delayed } \\
\text { due to pregnancy } \\
\text { induced hypertension } \\
\text { and other } \\
\text { complications after } \\
\text { childbirth } \\
\text { (performance of } \\
\text { post-birth activities) }\end{array}$ & $3 ; 10 ; 19$ & $\begin{array}{l}\text { Minor methodological } \\
\text { limitations (two studies } \\
\text { with minor concerns } \\
\text { and one study with } \\
\text { moderate concerns) }\end{array}$ & $\begin{array}{l}\text { Moderate concerns about } \\
\text { relevance (the review } \\
\text { finding is found in only } \\
\text { three studies: Chana, } \\
\text { Haiti and Malawi) }\end{array}$ & $\begin{array}{l}\text { Minor concerns about } \\
\text { coherence (data consistent } \\
\text { within and across } \\
\text { both studies) }\end{array}$ & $\begin{array}{l}\text { Substantial concerns about } \\
\text { adequacy (all studies show } \\
\text { poor data). }\end{array}$ & Moderate confidence & $\begin{array}{l}\text { This finding was graded as } \\
\text { moderate confidence because of } \\
\text { substantial concerns about } \\
\text { adequacy, moderate concerns } \\
\text { about relevance and minor } \\
\text { concerns about coherence and } \\
\text { methodological limitations. }\end{array}$ \\
\hline \multicolumn{9}{|c|}{$\begin{array}{c}\text { Barriers to Complementary Feeding } \\
\text { Kev Theme: Food Security and Social and Cultural F }\end{array}$} \\
\hline 39 & $\begin{array}{l}\text { Mothers and families } \\
\text { do not have enough } \\
\text { money to buy foods }\end{array}$ & $\begin{array}{c}11 ; 12 ; 14 ; 15 ; 16 ; 17 \\
20 ; 21 ; 23\end{array}$ & $\begin{array}{l}\text { Moderate } \\
\text { methodological } \\
\text { limitations (seven } \\
\text { studies with moderate } \\
\text { methodological } \\
\text { limitations and one } \\
\text { study with minor } \\
\text { limitations) }\end{array}$ & $\begin{array}{l}\text { Minor concerns about } \\
\text { relevance (the review } \\
\text { finding is relevant across } \\
\text { seven countries: Haiti, } \\
\text { Kenya, Democratic } \\
\text { Republic of Congo, } \\
\text { Ethiopia, Zambia and } \\
\text { Bangladesh) }\end{array}$ & $\begin{array}{l}\text { Minor concerns about } \\
\text { coherence (data consistent } \\
\text { within and across } \\
\text { both studies) }\end{array}$ & $\begin{array}{l}\text { Minor concerns about } \\
\text { adequacy (all studies provide } \\
\text { moderately rich data overall } \\
\text { except one study with } \\
\text { thin data) }\end{array}$ & High confidence & $\begin{array}{l}\text { This finding was graded as high } \\
\text { confidence because of minor } \\
\text { concerns about relevance, } \\
\text { coherence and adequacy; and } \\
\text { moderate methodological } \\
\text { limitations. }\end{array}$ \\
\hline 40 & $\begin{array}{l}\text { In periods of food } \\
\text { insecurity, especially } \\
\text { during drought, the } \\
\text { purchase of higher } \\
\text { quality foods are } \\
\text { replaced with } \\
\text { cheaper, less } \\
\text { nourishing foods }\end{array}$ & $12 ; 15$ & $\begin{array}{l}\text { Moderate } \\
\text { methodological } \\
\text { limitations (both } \\
\text { studies do not consider } \\
\text { potential bias. One } \\
\text { study does not state } \\
\text { analysis methods). }\end{array}$ & $\begin{array}{l}\text { Moderate concerns about } \\
\text { relevance (partial } \\
\text { relevance, as the studies } \\
\text { were from only two } \\
\text { settings: Kenya } \\
\text { and Ethiopia) }\end{array}$ & $\begin{array}{l}\text { Minor concerns about } \\
\text { coherence (data consistent } \\
\text { within and across } \\
\text { both studies) }\end{array}$ & $\begin{array}{l}\text { No concern about adequacy } \\
\text { of data (both studies provide } \\
\text { rich data) }\end{array}$ & Moderate confidence & $\begin{array}{l}\text { This finding was graded as } \\
\text { moderate confidence because of } \\
\text { minor concerns about coherence, } \\
\text { moderate concern about relevance } \\
\text { and methodological limitations }\end{array}$ \\
\hline 41 & $\begin{array}{l}\text { There are shortages of } \\
\text { diverse foods to meet } \\
\text { nutritional needs }\end{array}$ & $\begin{array}{c}12 ; 14 ; 15 ; 16 ; 17 ; 19 ; \\
20\end{array}$ & $\begin{array}{l}\text { Moderate } \\
\text { methodological } \\
\text { limitations (the } \\
\text { majority of studies with } \\
\text { moderate } \\
\text { methodological } \\
\text { limitations except with } \\
\text { one study with minor } \\
\text { concerns) }\end{array}$ & $\begin{array}{l}\text { Minor concerns about } \\
\text { relevance (the review } \\
\text { finding is relevant across } \\
\text { countries: Kenya, } \\
\text { Democratic Republic of } \\
\text { Congo, Ethiopia, Zambia } \\
\text { and Malawi) }\end{array}$ & $\begin{array}{l}\text { Minor concerns about } \\
\text { coherence (data consistent } \\
\text { within and across } \\
\text { both studies) }\end{array}$ & $\begin{array}{l}\text { Minor concerns about } \\
\text { adequacy (all studies provide } \\
\text { moderately rich data overall) }\end{array}$ & High confidence & $\begin{array}{l}\text { This finding was graded as high } \\
\text { confidence because of minor } \\
\text { concerns about relevance, } \\
\text { coherence and adequacy; and } \\
\text { moderate methodological } \\
\text { limitations. }\end{array}$ \\
\hline
\end{tabular}




\begin{tabular}{|c|c|c|c|c|c|c|c|c|}
\hline $\begin{array}{l}\text { Finding } \\
\text { No. }\end{array}$ & Review Finding & $\begin{array}{c}\text { Studies } \\
\text { Contributing to the } \\
\text { Review Finding }\end{array}$ & $\begin{array}{l}\text { Assessment of } \\
\text { Methodological } \\
\text { Limitations }\end{array}$ & $\begin{array}{l}\text { Assessment of } \\
\text { Relevance }\end{array}$ & Assessment of Coherence & Assessment of Adequacy & $\begin{array}{l}\text { Overall CERQual } \\
\text { Assessment of } \\
\text { Confidence }\end{array}$ & Explanation of Judgement \\
\hline 42 & $\begin{array}{l}\text { There is no piped } \\
\text { water in the houses } \\
\text { for cooking }\end{array}$ & $12 ; 15$ & $\begin{array}{l}\text { Moderate } \\
\text { methodological } \\
\text { limitations (both } \\
\text { studies do not consider } \\
\text { potential bias. One } \\
\text { study does not state } \\
\text { analysis methods). }\end{array}$ & $\begin{array}{l}\text { Moderate concerns about } \\
\text { relevance (partial } \\
\text { relevance, as the studies } \\
\text { were from only two } \\
\text { settings: Kenya } \\
\text { and Ethiopia) }\end{array}$ & $\begin{array}{l}\text { Minor concerns about } \\
\text { coherence (data consistent } \\
\text { within and across both } \\
\text { studies) }\end{array}$ & $\begin{array}{l}\text { No concern about adequacy } \\
\text { of data (both studies provide } \\
\text { rich data) }\end{array}$ & Moderate confidence & $\begin{array}{l}\text { This finding was graded as } \\
\text { moderate confidence because of } \\
\text { minor concerns about coherence, } \\
\text { moderate concern about relevance } \\
\text { and methodological limitations }\end{array}$ \\
\hline 43 & $\begin{array}{l}\text { Some foods are only } \\
\text { available seasonally }\end{array}$ & 12 & $\begin{array}{l}\text { Minor methodological } \\
\text { limitations (the authors } \\
\text { do not clarify } \\
\text { potential bias) }\end{array}$ & $\mathrm{NA}$ & NA & $\begin{array}{l}\text { Minor concern about } \\
\text { adequacy of data (the study } \\
\text { offers moderately rich data } \\
\text { overall) }\end{array}$ & NA & $\mathrm{NA}$ \\
\hline 44 & $\begin{array}{l}\text { Mothers prepare } \\
\text { foods early in the day } \\
\text { and then store it to } \\
\text { give to their } \\
\text { children later. }\end{array}$ & 12 & $\begin{array}{l}\text { Minor methodological } \\
\text { limitations (the authors } \\
\text { do not clarify } \\
\text { potential bias) }\end{array}$ & $\mathrm{NA}$ & NA & $\begin{array}{l}\text { No concern about adequacy } \\
\text { of data (the study provides } \\
\text { rich data) }\end{array}$ & $\mathrm{NA}$ & $\mathrm{NA}$ \\
\hline 45 & $\begin{array}{l}\text { Mothers do not have } \\
\text { financial } \\
\text { decision-making } \\
\text { power }\end{array}$ & $14 ; 20$ & $\begin{array}{l}\text { Moderate } \\
\text { methodological } \\
\text { limitations (both } \\
\text { studies do not consider } \\
\text { potential bias and } \\
\text { analysis methods. One } \\
\text { study does not } \\
\text { describe sampling) } \\
\end{array}$ & $\begin{array}{l}\text { Moderate concerns about } \\
\text { relevance (partial } \\
\text { relevance, as the studies } \\
\text { were from only two } \\
\text { settings: Democratic } \\
\text { Republic of Congo } \\
\text { and Ethiopia) }\end{array}$ & $\begin{array}{l}\text { Minor concerns about } \\
\text { coherence (data consistent } \\
\text { within and across both } \\
\text { studies) }\end{array}$ & $\begin{array}{l}\text { No concern about adequacy } \\
\text { of data (both studies offer } \\
\text { rich data) }\end{array}$ & Moderate confidence & $\begin{array}{l}\text { This finding was graded as } \\
\text { moderate confidence because of } \\
\text { minor concerns about coherence, } \\
\text { moderate concern about relevance } \\
\text { and methodological limitations }\end{array}$ \\
\hline \multicolumn{9}{|c|}{ Key Theme: Infant-Specific Factors } \\
\hline 46 & $\begin{array}{l}\text { Infants dislike animal } \\
\text { source foods }\end{array}$ & $6 ; 23$ & $\begin{array}{l}\text { Moderate } \\
\text { methodological } \\
\text { limitations (both } \\
\text { studies do not consider } \\
\text { potential bias and } \\
\text { analysis methods.) }\end{array}$ & $\begin{array}{l}\text { Moderate concerns about } \\
\text { relevance (partial } \\
\text { relevance, as the studies } \\
\text { were from only one } \\
\text { country: Bangladesh) }\end{array}$ & $\begin{array}{l}\text { Minor concerns about } \\
\text { coherence (data consistent } \\
\text { within and across } \\
\text { both studies) }\end{array}$ & $\begin{array}{l}\text { Substantial concern about } \\
\text { adequacy (both studies } \\
\text { provide thin data) }\end{array}$ & Low confidence & $\begin{array}{l}\text { This finding was graded as low } \\
\text { confidence because of moderate } \\
\text { concerns about methodology and } \\
\text { relevance; and substantial } \\
\text { concerns about adequacy of data. }\end{array}$ \\
\hline 47 & $\begin{array}{l}\text { Infants do not have } \\
\text { appetite to eat animal } \\
\text { source foods }\end{array}$ & $6 ; 18$ & $\begin{array}{l}\text { Moderate } \\
\text { methodological } \\
\text { limitations (the authors } \\
\text { in both studies do not } \\
\text { consider potential bias. } \\
\text { One study does not } \\
\text { describe sampling. The } \\
\text { other one does not } \\
\text { explain } \\
\text { analysis methods.) }\end{array}$ & $\begin{array}{l}\text { Moderate concerns about } \\
\text { relevance (partial } \\
\text { relevance, as the studies } \\
\text { were from only two } \\
\text { settings: Bangladesh } \\
\text { and Nigeria) }\end{array}$ & $\begin{array}{l}\text { Minor concerns about } \\
\text { coherence (data consistent } \\
\text { within and across } \\
\text { both studies) }\end{array}$ & $\begin{array}{l}\text { Moderate concern about } \\
\text { adequacy (One study } \\
\text { provides rich data. The other } \\
\text { one offers only thin data) }\end{array}$ & Moderate confidence & $\begin{array}{l}\text { This finding was graded as } \\
\text { moderate confidence because of } \\
\text { moderate concerns regarding } \\
\text { methodological limitations, } \\
\text { adequacy of data, relevance and } \\
\text { minor concerns about coherence. }\end{array}$ \\
\hline
\end{tabular}




\begin{tabular}{|c|c|c|c|c|c|c|c|c|}
\hline $\begin{array}{l}\text { Finding } \\
\text { No. }\end{array}$ & Review Finding & $\begin{array}{c}\text { Studies } \\
\text { Contributing to the } \\
\text { Review Finding }\end{array}$ & $\begin{array}{c}\text { Assessment of } \\
\text { Methodological } \\
\text { Limitations }\end{array}$ & $\begin{array}{l}\text { Assessment of } \\
\text { Relevance }\end{array}$ & Assessment of Coherence & Assessment of Adequacy & $\begin{array}{l}\text { Overall CERQual } \\
\text { Assessment of } \\
\text { Confidence }\end{array}$ & Explanation of Judgement \\
\hline 48 & $\begin{array}{l}\text { Infants refuse to eat } \\
\text { and or spit out } \\
\text { of foods }\end{array}$ & $6 ; 15 ; 23$ & $\begin{array}{l}\text { Moderate } \\
\text { methodological } \\
\text { limitations (all studies } \\
\text { with moderate } \\
\text { methodological } \\
\text { limitations) }\end{array}$ & $\begin{array}{l}\text { Moderate concerns about } \\
\text { relevance (partial } \\
\text { relevance, as the studies } \\
\text { were from only two } \\
\text { settings: Bangladesh } \\
\text { and Ethiopia) }\end{array}$ & $\begin{array}{l}\text { Moderate concern about } \\
\text { coherence (data partially } \\
\text { consistent within and across } \\
\text { both studies) }\end{array}$ & $\begin{array}{l}\text { Substantial concerns about } \\
\text { adequacy (all studies offer } \\
\text { thin data) }\end{array}$ & Low confidence & $\begin{array}{l}\text { This finding was graded as low } \\
\text { confidence because of moderate } \\
\text { concerns about relevance and } \\
\text { coherence and substantial } \\
\text { concerns about adequacy of data } \\
\text { and methodology. }\end{array}$ \\
\hline 49 & $\begin{array}{l}\text { Infants vomit when } \\
\text { animal source foods } \\
\text { are offered }\end{array}$ & $6 ; 15$ & $\begin{array}{l}\text { Substantial } \\
\text { methodological } \\
\text { limitations (the authors } \\
\text { in both studies do not } \\
\text { consider potential bias } \\
\text { and analysis methods. } \\
\text { One study does not } \\
\text { describe sampling.) }\end{array}$ & $\begin{array}{l}\text { Moderate concerns about } \\
\text { relevance (partial } \\
\text { relevance, as the studies } \\
\text { were from only two } \\
\text { settings: Bangladesh } \\
\text { and Ethiopia) }\end{array}$ & $\begin{array}{l}\text { Moderate concern about } \\
\text { coherence (data partially } \\
\text { consistent within and across } \\
\text { both studies) }\end{array}$ & $\begin{array}{l}\text { Substantial concerns about } \\
\text { adequacy (only two studies, } \\
\text { both offering thin data) }\end{array}$ & Low confidence & $\begin{array}{l}\text { This finding was graded as low } \\
\text { confidence because of moderate } \\
\text { concerns about relevance and } \\
\text { coherence and substantial } \\
\text { concerns about adequacy of data } \\
\text { and methodology. }\end{array}$ \\
\hline 50 & $\begin{array}{l}\text { Infants have } \\
\text { a sickness }\end{array}$ & $15 ; 18$ & $\begin{array}{l}\text { Moderate } \\
\text { methodological } \\
\text { limitations (the authors } \\
\text { in both studies do not } \\
\text { consider potential bias } \\
\text { and analysis methods.) }\end{array}$ & $\begin{array}{l}\text { Moderate concerns about } \\
\text { relevance (partial } \\
\text { relevance, as the studies } \\
\text { were from only two } \\
\text { settings: Nigeria and } \\
\text { Ethiopia) }\end{array}$ & $\begin{array}{l}\text { Minor concerns about } \\
\text { coherence (data consistent } \\
\text { within and across } \\
\text { both studies) }\end{array}$ & $\begin{array}{l}\text { No concern about adequacy } \\
\text { of data (both studies offer } \\
\text { rich data) }\end{array}$ & Moderate confidence & $\begin{array}{l}\text { This finding was graded as } \\
\text { moderate confidence because of } \\
\text { moderate concerns about methods } \\
\text { and methodology and minor } \\
\text { concerns about coherence. }\end{array}$ \\
\hline 51 & $\begin{array}{l}\text { Mothers and other } \\
\text { family members do } \\
\text { not have knowledge } \\
\text { about complementary } \\
\text { feeding }\end{array}$ & $\begin{array}{c}6 ; 11 ; 14 ; 15 ; 16 ; 19 ; 20 \\
21 ; 23\end{array}$ & $\begin{array}{l}\text { Moderate } \\
\text { methodological } \\
\text { limitations (all studies } \\
\text { with moderate } \\
\text { methodological } \\
\text { limitations) }\end{array}$ & $\begin{array}{l}\text { Key Them } \\
\text { Minor concerns about } \\
\text { relevance (the review } \\
\text { finding is relevant in six } \\
\text { countries: Bangladesh, } \\
\text { Haiti, Democratic } \\
\text { Republic of Congo, } \\
\text { Ethiopia, Malawi } \\
\text { and Kenya) }\end{array}$ & $\begin{array}{l}\text { Minor concerns about } \\
\text { coherence (data consistent } \\
\text { within and across } \\
\text { both studies) }\end{array}$ & $\begin{array}{l}\text { Minor concerns about } \\
\text { adequacy (two studies offer } \\
\text { only thin data, but other } \\
\text { studies provide rich data } \\
\text { overall) }\end{array}$ & High confidence & $\begin{array}{l}\text { This finding was graded as high } \\
\text { confidence because of minor } \\
\text { concerns about relevance, } \\
\text { coherence and adequacy; and } \\
\text { moderate methodological } \\
\text { limitations. }\end{array}$ \\
\hline 52 & $\begin{array}{l}\text { Mothers are } \\
\text { concerned about } \\
\text { over-feeding } \\
\text { their child }\end{array}$ & 16 & $\begin{array}{l}\text { Moderate } \\
\text { methodological } \\
\text { limitations (the study } \\
\text { does not consider } \\
\text { potential bias and } \\
\text { analysis methods) }\end{array}$ & NA & NA & $\begin{array}{l}\text { No concern about adequacy } \\
\text { of data (the study provides } \\
\text { rich data) }\end{array}$ & NA & NA \\
\hline 53 & $\begin{array}{l}\text { Mothers and family } \\
\text { members have fear } \\
\text { that child may choke } \\
\text { on certain solid foods }\end{array}$ & $15 ; 16 ; 19$ & $\begin{array}{l}\text { Moderate } \\
\text { methodological } \\
\text { limitations (all studies } \\
\text { do not consider } \\
\text { potential bias. Two } \\
\text { studies do not explain } \\
\text { analysis methods) }\end{array}$ & $\begin{array}{l}\text { Moderate concerns about } \\
\text { relevance (partial } \\
\text { relevance, as the studies } \\
\text { were from only three } \\
\text { settings: Malawi and } \\
\text { SNNP and } \\
\text { Tigrey Ethiopia) }\end{array}$ & $\begin{array}{l}\text { Minor concerns about } \\
\text { coherence (data consistent } \\
\text { within and across } \\
\text { both studies) }\end{array}$ & $\begin{array}{l}\text { Moderate concern about } \\
\text { adequacy of data (one study } \\
\text { provides moderately rich } \\
\text { data overall, but other } \\
\text { studies offer only thin data) }\end{array}$ & Moderate confidence & $\begin{array}{l}\text { This finding was graded as } \\
\text { moderate confidence because of } \\
\text { moderate concerns about } \\
\text { methodology, adequacy of data, } \\
\text { relevance and minor concern } \\
\text { regarding coherence. }\end{array}$ \\
\hline
\end{tabular}




\begin{tabular}{|c|c|c|c|c|c|c|c|c|}
\hline $\begin{array}{l}\text { Finding } \\
\text { No. }\end{array}$ & Review Finding & $\begin{array}{c}\text { Studies } \\
\text { Contributing to the } \\
\text { Review Finding }\end{array}$ & $\begin{array}{c}\text { Assessment of } \\
\text { Methodological } \\
\text { Limitations }\end{array}$ & $\begin{array}{l}\text { Assessment of } \\
\text { Relevance }\end{array}$ & Assessment of Coherence & Assessment of Adequacy & $\begin{array}{l}\text { Overall CERQual } \\
\text { Assessment of } \\
\text { Confidence }\end{array}$ & Explanation of Judgement \\
\hline 54 & $\begin{array}{l}\text { Mothers and other } \\
\text { family members are } \\
\text { not willing to prepare } \\
\text { non-fasting foods for } \\
\text { children }\end{array}$ & 15 & $\begin{array}{l}\text { Moderate } \\
\text { methodological } \\
\text { limitations (the study } \\
\text { does not consider } \\
\text { potential bias and } \\
\text { analysis methods) }\end{array}$ & NA & NA & $\begin{array}{l}\text { Moderate concern about } \\
\text { adequacy of data (the study } \\
\text { provides only thin data) }\end{array}$ & NA & NA \\
\hline 55 & $\begin{array}{l}\text { Thick porridge is } \\
\text { believed to be } \\
\text { difficult for young } \\
\text { children to digest and } \\
\text { thus avoided }\end{array}$ & $15 ; 16 ; 19 ; 20 ; 23$ & $\begin{array}{l}\text { Moderate } \\
\text { methodological } \\
\text { limitations (all studies } \\
\text { with moderate } \\
\text { methodological } \\
\text { limitations) }\end{array}$ & $\begin{array}{l}\text { Moderate concerns about } \\
\text { relevance (partial } \\
\text { relevance, as the studies } \\
\text { were from only three } \\
\text { countries: Malawi, } \\
\text { Ethiopia and Bangladesh) }\end{array}$ & $\begin{array}{l}\text { Minor concerns about } \\
\text { coherence (data consistent } \\
\text { within and across } \\
\text { both studies) }\end{array}$ & $\begin{array}{l}\text { Moderate concern about } \\
\text { adequacy of data (one study } \\
\text { provides moderately rich } \\
\text { data overall, but other } \\
\text { studies offer only thin data) }\end{array}$ & Moderate confidence & $\begin{array}{l}\text { This finding was graded as } \\
\text { moderate confidence because of } \\
\text { moderate concerns about } \\
\text { methodology, adequacy of data, } \\
\text { relevance and minor concern } \\
\text { regarding coherence. }\end{array}$ \\
\hline 56 & $\begin{array}{l}\text { Mothers and family } \\
\text { members do not have } \\
\text { enought time to cook } \\
\text { for their child }\end{array}$ & $15 ; 16$ & $\begin{array}{l}\text { Moderate } \\
\text { methodological } \\
\text { limitations (both } \\
\text { studies do not consider } \\
\text { potential bias and } \\
\text { analysis methods) } \\
\end{array}$ & $\begin{array}{l}\text { Moderate concerns about } \\
\text { relevance (partial } \\
\text { relevance, as the studies } \\
\text { were from only two } \\
\text { settings: Tigrey and } \\
\text { SNNP Ethiopia) } \\
\end{array}$ & $\begin{array}{l}\text { Minor concerns about } \\
\text { coherence (data consistent } \\
\text { within and across } \\
\text { both studies) }\end{array}$ & $\begin{array}{l}\text { Minor concern about } \\
\text { adequacy of data (both } \\
\text { studies provide moderately } \\
\text { rich data overall) }\end{array}$ & Moderate confidence & $\begin{array}{l}\text { This finding was graded as } \\
\text { moderate confidence because of } \\
\text { moderate concerns about } \\
\text { methodology and relevance and } \\
\text { minor concerns regarding } \\
\text { coherence and adequacy of data. }\end{array}$ \\
\hline \multicolumn{9}{|c|}{$\begin{array}{lll} & \text { Cross-Cutting Barriers } \\
\end{array}$} \\
\hline 57 & $\begin{array}{l}\text { Inability of HWs } \\
\text { to support }\end{array}$ & 15 & $\begin{array}{l}\text { Moderate } \\
\text { methodological } \\
\text { limitations (the authors } \\
\text { do not consider } \\
\text { potential bias and } \\
\text { analysis methods) }\end{array}$ & $\mathrm{NA}$ & NA & $\begin{array}{l}\text { No concerns about adequacy } \\
\text { of data (the study provides } \\
\text { rich data) }\end{array}$ & NA & NA \\
\hline 58 & $\begin{array}{l}\text { Conflicting } \\
\text { information }\end{array}$ & 21 & $\begin{array}{l}\text { Moderate } \\
\text { methodological } \\
\text { limitations (the authors } \\
\text { do not explain potential } \\
\text { bias and sampling } \\
\text { methods) }\end{array}$ & NA & NA & $\begin{array}{l}\text { Moderate concerns (the } \\
\text { study offers thin data) }\end{array}$ & NA & NA \\
\hline \multirow{2}{*}{\multicolumn{9}{|c|}{$\begin{array}{l}\text { Facilitators } \\
\text { Facilitators to Recommended Breastfeeding }\end{array}$}} \\
\hline \multicolumn{7}{|c|}{ Key Theme: Perceptions } & & \\
\hline 59 & $\begin{array}{l}\text { Breastfeeding is a } \\
\text { cheap and hygienic } \\
\text { way of feeding }\end{array}$ & $1 ; 2 ; 4 ; 7 ; 9 ; 13$ & $\begin{array}{l}\text { Moderate } \\
\text { methodological } \\
\text { limitations (all studies } \\
\text { with moderate } \\
\text { methodological } \\
\text { limitations) }\end{array}$ & $\begin{array}{l}\text { Minor concerns about } \\
\text { relevance (the review } \\
\text { fining is relevant in six } \\
\text { countries: Dominican } \\
\text { Republic, Tanzania, } \\
\text { Ghana, Vietnam, Lao } \\
\text { PDR and Cambodia) }\end{array}$ & $\begin{array}{l}\text { Minor concerns about } \\
\text { coherence (data consistent } \\
\text { within and across } \\
\text { both studies) }\end{array}$ & $\begin{array}{l}\text { Minor concerns about } \\
\text { adequacy (four studies } \\
\text { provide rich data, but two } \\
\text { studies show only thin data) }\end{array}$ & High confidence & $\begin{array}{l}\text { This finding was graded as high } \\
\text { confidence because of minor } \\
\text { concerns about relevance, } \\
\text { coherence and adequacy; and } \\
\text { moderate methodological } \\
\text { limitations. }\end{array}$ \\
\hline
\end{tabular}




\begin{tabular}{|c|c|c|c|c|c|c|c|c|}
\hline $\begin{array}{l}\text { Finding } \\
\text { No. }\end{array}$ & Review Finding & $\begin{array}{c}\text { Studies } \\
\text { Contributing to the } \\
\text { Review Finding }\end{array}$ & $\begin{array}{l}\text { Assessment of } \\
\text { Methodological } \\
\text { Limitations }\end{array}$ & $\begin{array}{l}\text { Assessment of } \\
\text { Relevance }\end{array}$ & Assessment of Coherence & Assessment of Adequacy & $\begin{array}{l}\text { Overall CERQual } \\
\text { Assessment of } \\
\text { Confidence }\end{array}$ & Explanation of Judgement \\
\hline 60 & $\begin{array}{l}\text { Breastfeeding relieves } \\
\text { uncomfortable } \\
\text { fullness of breasts }\end{array}$ & 4 & $\begin{array}{l}\text { Moderate } \\
\text { methodological } \\
\text { limitations (the study } \\
\text { does not state analysis } \\
\text { methods and } \\
\text { potential bias) }\end{array}$ & NA & NA & $\begin{array}{l}\text { Moderate concerns about } \\
\text { adequacy (the authors } \\
\text { provide only thin data) }\end{array}$ & NA & NA \\
\hline 61 & $\begin{array}{l}\text { Breastfeeding } \\
\text { strengthens the bond } \\
\text { between the mother } \\
\text { and child }\end{array}$ & $5 ; 9$ & $\begin{array}{l}\text { Moderate } \\
\text { methodological } \\
\text { limitations (both } \\
\text { studies do not consider } \\
\text { potential bias. One } \\
\text { does not state analysis } \\
\text { methods. The other one } \\
\text { does not describe } \\
\text { sampling) }\end{array}$ & $\begin{array}{l}\text { Moderate concerns about } \\
\text { relevance (partial } \\
\text { relevance as the studies } \\
\text { were from only two } \\
\text { settings: Colombia and } \\
\text { Laos PDR) }\end{array}$ & $\begin{array}{l}\text { Minor concerns about } \\
\text { coherence (data consistent } \\
\text { within and across both } \\
\text { studies) }\end{array}$ & $\begin{array}{l}\text { Moderate concerns about } \\
\text { adequacy (one study } \\
\text { provides only thin data. The } \\
\text { other one provides rich data) }\end{array}$ & Moderate confidence & $\begin{array}{l}\text { This finding was graded as } \\
\text { moderate confidence because of } \\
\text { moderate concerns regarding } \\
\text { methodology, relevance and } \\
\text { adequacy and minor concerns } \\
\text { about coherence. }\end{array}$ \\
\hline \multicolumn{9}{|c|}{ Key Theme: Healthcare Services } \\
\hline 62 & $\begin{array}{l}\text { Mothers and family } \\
\text { members received } \\
\text { advice and } \\
\text { encouragement at } \\
\text { healthcare setting(s) } \\
\text { (e.g., prenatal visits, } \\
\text { delivery, health posts, } \\
\text { health worker } \\
\text { home visits) }\end{array}$ & $3 ; 10 ; 13 ; 16 ; 17 ; 18$ & $\begin{array}{l}\text { Moderate } \\
\text { methodological } \\
\text { limitations (five studies } \\
\text { with moderate } \\
\text { methodological } \\
\text { limitations, one study } \\
\text { with minor limitations) }\end{array}$ & $\begin{array}{l}\text { Minor concerns about } \\
\text { relevance e the review } \\
\text { fining is relevant in six } \\
\text { countries: Ghana, Haiti, } \\
\text { Cambodia, Ethiopia, } \\
\text { Zambia and Nigeria) }\end{array}$ & $\begin{array}{l}\text { Minor concerns about } \\
\text { coherence (data consistent } \\
\text { within and across } \\
\text { both studies) }\end{array}$ & $\begin{array}{l}\text { Substantial concerns about } \\
\text { adequacy (four studies show } \\
\text { poor data. Too studies show } \\
\text { moderately rich data) }\end{array}$ & Moderate confidence & $\begin{array}{l}\text { This finding was graded as } \\
\text { moderate confidence because of } \\
\text { substantial concerns about } \\
\text { adequacy, moderate } \\
\text { methodological limitations, minor } \\
\text { concerns about relevance and } \\
\text { coherence. }\end{array}$ \\
\hline 63 & $\begin{array}{l}\text { Mothers and family } \\
\text { members receive } \\
\text { advice and } \\
\text { counseling from HWs }\end{array}$ & $\begin{array}{c}2 ; 3 ; 4 ; 9 ; 13 ; 14 ; 15 ; 16 ; \\
17 ; 18 ; 19\end{array}$ & $\begin{array}{l}\text { Moderate } \\
\text { methodological } \\
\text { limitations (ten studies } \\
\text { with moderate } \\
\text { methodological } \\
\text { limitations, one study } \\
\text { with minor limitations) }\end{array}$ & $\begin{array}{l}\text { Minor concerns about } \\
\text { relevance (the review } \\
\text { fining is universal in six } \\
\text { countries: Tanzania, } \\
\text { Ghana, Lao PDR, } \\
\text { Cambodia, Democratic } \\
\text { Republic of Congo, } \\
\text { Ethiopia, Zambia, } \\
\text { Nigeria and Malawi) }\end{array}$ & $\begin{array}{l}\text { Minor concerns about } \\
\text { coherence (data consistent } \\
\text { within and across } \\
\text { both studies) }\end{array}$ & $\begin{array}{l}\text { Substantial concerns about } \\
\text { adequacy (four studies } \\
\text { provide moderately rich data. } \\
\text { Other studies offer only } \\
\text { thin data) }\end{array}$ & Moderate confidence & $\begin{array}{l}\text { This finding was graded as } \\
\text { moderate confidence because of } \\
\text { substantial concerns about } \\
\text { adequacy, moderate } \\
\text { methodological limitations, minor } \\
\text { concerns about relevance and } \\
\text { coherence. }\end{array}$ \\
\hline \multicolumn{9}{|c|}{ Key Theme: $\operatorname{In}$} \\
\hline 64 & $\begin{array}{l}\text { Crying provides } \\
\text { guidance for baby } \\
\text { feeding practices }\end{array}$ & $2 ; 10$ & $\begin{array}{l}\text { Minor methodological } \\
\text { limitations (one study } \\
\text { does not state sampling } \\
\text { methods and } \\
\text { potential bias) }\end{array}$ & $\begin{array}{l}\text { Moderate concerns about } \\
\text { relevance (partial } \\
\text { relevance as the studies } \\
\text { were from only two } \\
\text { settings: Tanzania } \\
\text { and Haiti) }\end{array}$ & $\begin{array}{l}\text { Minor concerns about } \\
\text { coherence (data consistent } \\
\text { within and across } \\
\text { both studies) }\end{array}$ & $\begin{array}{l}\text { Minor concerns about } \\
\text { adequacy (both studies } \\
\text { provide rich data overall) }\end{array}$ & High confidence & $\begin{array}{l}\text { This finding was graded as high } \\
\text { confidence due to only minor } \\
\text { concerns about adequacy, } \\
\text { relevance and methodological } \\
\text { limitations and moderate concerns } \\
\text { about relevance. }\end{array}$ \\
\hline
\end{tabular}




\begin{tabular}{|c|c|c|c|c|c|c|c|c|}
\hline $\begin{array}{l}\text { Finding } \\
\text { No. }\end{array}$ & Review Finding & $\begin{array}{c}\text { Studies } \\
\text { Contributing to the } \\
\text { Review Finding }\end{array}$ & $\begin{array}{c}\text { Assessment of } \\
\text { Methodological } \\
\text { Limitations }\end{array}$ & $\begin{array}{l}\text { Assessment of } \\
\text { Relevance }\end{array}$ & Assessment of Coherence & Assessment of Adequacy & $\begin{array}{l}\text { Overall CERQual } \\
\text { Assessment of } \\
\text { Confidence }\end{array}$ & Explanation of Judgement \\
\hline \multicolumn{9}{|c|}{ Key Theme: Mother-Specific Factors } \\
\hline 65 & $\begin{array}{l}\text { Mothers understand } \\
\text { the importance of } \\
\text { breastfeeding }\end{array}$ & $\begin{array}{c}1 ; 2 ; 3 ; 4 ; 5 ; 7 ; 9 ; 10 ; 11 ; \\
15 ; 16 ; 17 ; 18 ; 19 ; 21 \\
22\end{array}$ & $\begin{array}{l}\text { Moderate } \\
\text { methodological } \\
\text { limitations }(14 \text { studies } \\
\text { with moderate } \\
\text { methodological } \\
\text { limitations, two studies } \\
\text { with minor limitations) }\end{array}$ & $\begin{array}{l}\text { Minor concerns about } \\
\text { relevance (the review } \\
\text { fining is universal in } 12 \\
\text { countries: Dominican } \\
\text { Republic, Tanzania, } \\
\text { Ghana, Colombia, } \\
\text { Vietnam, Lao PDR, Haiti, } \\
\text { Ethiopia, Zambia, } \\
\text { Nigeria, Malawi } \\
\text { and Kenya) }\end{array}$ & $\begin{array}{l}\text { Minor concerns about } \\
\text { coherence (data consistent } \\
\text { within and across } \\
\text { both studies) }\end{array}$ & $\begin{array}{l}\text { Minor concerns about } \\
\text { adequacy (the majority of } \\
\text { studies provide rich } \\
\text { information while three } \\
\text { studies offer only thin data) }\end{array}$ & High confidence & $\begin{array}{l}\text { This finding was graded as high } \\
\text { confidence because of minor } \\
\text { concerns about relevance, } \\
\text { coherence and adequacy; and } \\
\text { moderate } \\
\text { methodological limitations. }\end{array}$ \\
\hline 66 & $\begin{array}{l}\text { Mothers understand } \\
\text { the importance } \\
\text { of colostrum }\end{array}$ & $2 ; 7 ; 14 ; 19$ & $\begin{array}{l}\text { Moderate } \\
\text { methodological } \\
\text { limitations (all studies } \\
\text { with moderate } \\
\text { methodological } \\
\text { limitations) }\end{array}$ & $\begin{array}{l}\text { Minor concerns about } \\
\text { relevance (the review } \\
\text { finding is relevant in four } \\
\text { countries: Tanzania, } \\
\text { Vietnam, Democratic } \\
\text { Republic of Congo } \\
\text { and Malawi) }\end{array}$ & $\begin{array}{l}\text { Minor concerns about } \\
\text { coherence (data consistent } \\
\text { within and across both } \\
\text { studies) }\end{array}$ & $\begin{array}{l}\text { Minor concerns about } \\
\text { adequacy (all studies provide } \\
\text { moderately rich data overall) }\end{array}$ & High confidence & $\begin{array}{l}\text { This finding was graded as high } \\
\text { confidence because of minor } \\
\text { concerns about relevance, } \\
\text { coherence and adequacy; and } \\
\text { moderate } \\
\text { methodological limitations. }\end{array}$ \\
\hline \multicolumn{9}{|c|}{ Key Theme: Social and Cultural Factors } \\
\hline 67 & $\begin{array}{l}\text { Cultures and } \\
\text { ethnicity facilitate } \\
\text { early initiation } \\
\text { of breastfeeding }\end{array}$ & 3 & $\begin{array}{l}\text { Moderate } \\
\text { methodological } \\
\text { limitations (the authors } \\
\text { do not explain analysis } \\
\text { methods and } \\
\text { potential bias) }\end{array}$ & NA & NA & $\begin{array}{l}\text { No concerns about adequacy } \\
\text { (the study provides rich data) }\end{array}$ & NA & NA \\
\hline 68 & $\begin{array}{l}\text { Family support } \\
\text { promotes } \\
\text { breastfeeding }\end{array}$ & $1 ; 2 ; 7 ; 9$ & $\begin{array}{l}\text { Moderate } \\
\text { methodological } \\
\text { limitations (all studies } \\
\text { with moderate } \\
\text { methodological } \\
\text { limitations) }\end{array}$ & $\begin{array}{l}\text { Minor concerns about } \\
\text { relevance (the review } \\
\text { finding is relevant in four } \\
\text { countries: Dominican } \\
\text { Republic, Tanzania, } \\
\text { Vietnam and Lao PDR) }\end{array}$ & $\begin{array}{l}\text { Minor concerns about } \\
\text { coherence (data consistent } \\
\text { within and across } \\
\text { both studies) }\end{array}$ & $\begin{array}{l}\text { Substantial concerns about } \\
\text { adequacy (only one study } \\
\text { provides rich data. Other } \\
\text { studies show poor data) }\end{array}$ & Moderate confidence & $\begin{array}{l}\text { This finding was graded as } \\
\text { moderate confidence because of } \\
\text { moderate methodological } \\
\text { limitations, minor concerns } \\
\text { regarding relevance and coherence } \\
\text { and substantial concerns } \\
\text { about adequacy. }\end{array}$ \\
\hline 69 & $\begin{array}{l}\text { Praise and } \\
\text { affirmation from a } \\
\text { partner and/or family } \\
\text { facilitate } \\
\text { breastfeeding }\end{array}$ & 2 & $\begin{array}{l}\text { Moderate } \\
\text { methodological } \\
\text { limitations (the authors } \\
\text { do not explain } \\
\text { sampling and } \\
\text { potential bias) }\end{array}$ & $\mathrm{NA}$ & $\mathrm{NA}$ & $\begin{array}{l}\text { No concerns about adequacy } \\
\text { (the study provides rich data) }\end{array}$ & $\mathrm{NA}$ & $\mathrm{NA}$ \\
\hline \multicolumn{9}{|c|}{$\begin{array}{l}\text { Facilitators to Complementary Feeding } \\
\text { Key Theme: Food Security }\end{array}$} \\
\hline 70 & $\begin{array}{l}\text { Some foods are } \\
\text { available }\end{array}$ & $16 ; 18 ; 22$ & $\begin{array}{l}\text { Moderate } \\
\text { methodological } \\
\text { limitations (all studies } \\
\text { with moderate } \\
\text { methodological } \\
\text { limitations) }\end{array}$ & $\begin{array}{l}\text { Moderate concerns about } \\
\text { relevance (partial } \\
\text { relevance as the studies } \\
\text { are from only three } \\
\text { settings: } \\
\text { Ethiopia, Nigeria } \\
\text { and Ghana) }\end{array}$ & $\begin{array}{l}\text { Minor concerns about } \\
\text { coherence (data consistent } \\
\text { within and across } \\
\text { both studies) }\end{array}$ & $\begin{array}{l}\text { Minor concerns about } \\
\text { adequacy (all studies provide } \\
\text { moderately rich data overall) }\end{array}$ & Moderate confidence & $\begin{array}{l}\text { This finding was graded as } \\
\text { moderate confidence because of } \\
\text { moderate concerns about } \\
\text { methodology and relevance; and } \\
\text { minor concerns about coherence } \\
\text { and adequacy. }\end{array}$ \\
\hline
\end{tabular}




\begin{tabular}{|c|c|c|c|c|c|c|c|c|}
\hline $\begin{array}{l}\text { Finding } \\
\text { No. }\end{array}$ & Review Finding & $\begin{array}{l}\text { Studies } \\
\text { Contributing to the } \\
\text { Review Finding }\end{array}$ & $\begin{array}{l}\text { Assessment of } \\
\text { Methodological } \\
\text { Limitations }\end{array}$ & $\begin{array}{l}\text { Assessment of } \\
\text { Relevance }\end{array}$ & Assessment of Coherence & Assessment of Adequacy & $\begin{array}{l}\text { Overall CERQual } \\
\text { Assessment of } \\
\text { Confidence }\end{array}$ & Explanation of Judgement \\
\hline \multicolumn{9}{|c|}{ Key Theme: Social Support } \\
\hline 71 & $\begin{array}{l}\text { Family support } \\
\text { promotes } \\
\text { complementary } \\
\text { feeding }\end{array}$ & $6 ; 15 ; 16 ; 21$ & $\begin{array}{l}\text { methodological } \\
\text { limitations (all studies } \\
\text { with moderate } \\
\text { methodological } \\
\text { limitations) }\end{array}$ & $\begin{array}{l}\text { relevance (partial } \\
\text { relevance as the studies } \\
\text { are from only three } \\
\text { countries: Bangladesh, } \\
\text { Ethiopia and Kenya) }\end{array}$ & $\begin{array}{l}\text { Minor concerns about } \\
\text { coherence (data consistent } \\
\text { within and across } \\
\text { both studies) }\end{array}$ & $\begin{array}{l}\text { Minor concerns about } \\
\text { adequacy (three studies } \\
\text { provide rich data, but one } \\
\text { study offers only thin data) }\end{array}$ & Moderate confidence & $\begin{array}{l}\text { moderate confidence because of } \\
\text { minor concerns about coherence } \\
\text { and adequacy; and moderate } \\
\text { concerns regarding relevance } \\
\text { and methodology. }\end{array}$ \\
\hline \multicolumn{9}{|c|}{ Key Theme: Mother-Specific Factors } \\
\hline 72 & $\begin{array}{l}\text { Mothers understand } \\
\text { the importance of } \\
\text { complementary } \\
\text { feeding }\end{array}$ & $15 ; 16 ; 18$ & $\begin{array}{l}\text { Moderate } \\
\text { methodological } \\
\text { limitations (all studies } \\
\text { with moderate } \\
\text { methodological } \\
\text { limitations) }\end{array}$ & $\begin{array}{l}\text { Moderate concerns about } \\
\text { relevance (partial } \\
\text { relevance as the studies } \\
\text { are from only two } \\
\text { countries, Ethiopia } \\
\text { and Nigeria) }\end{array}$ & $\begin{array}{l}\text { Minor concerns about } \\
\text { coherence (data consistent } \\
\text { within and across } \\
\text { both studies) }\end{array}$ & $\begin{array}{l}\text { Minor concerns about } \\
\text { adequacy (all studies provide } \\
\text { rich data) }\end{array}$ & Moderate confidence & $\begin{array}{l}\text { This finding was graded as } \\
\text { moderate confidence because of } \\
\text { minor concerns about coherence } \\
\text { and adequacy; and } \\
\text { moderate concerns }\end{array}$ \\
\hline \multicolumn{9}{|c|}{ Key Theme: Healthcare Services } \\
\hline 73 & $\begin{array}{l}\text { Mothers receive IYCF } \\
\text { advice and } \\
\text { counseling at } \\
\text { healthcare facilities }\end{array}$ & $15 ; 18 ; 19 ; 21$ & $\begin{array}{l}\text { Moderate } \\
\text { methodological } \\
\text { limitations (all studies } \\
\text { with moderate } \\
\text { methodological } \\
\text { limitations) }\end{array}$ & $\begin{array}{l}\text { Minor concerns about } \\
\text { relevancece (the review } \\
\text { findings is relevant in } \\
\text { four countries: Ethiopia, } \\
\text { Nigeria, Malawi } \\
\text { and Kenya) }\end{array}$ & $\begin{array}{l}\text { Minor concerns about } \\
\text { coherence (data consistent } \\
\text { within and across } \\
\text { both studies) }\end{array}$ & $\begin{array}{l}\text { Substantial concerns about } \\
\text { adequacy (three studies } \\
\text { provide thin data. Only one } \\
\text { study shows moderately } \\
\text { rich data) }\end{array}$ & Moderate confidence & $\begin{array}{l}\text { This finding was graded as } \\
\text { moderate confidence because of } \\
\text { minor concerns about coherence } \\
\text { and adequacy; and moderate } \\
\text { methodological limitations and } \\
\text { substantial concerns } \\
\text { about adequacy. }\end{array}$ \\
\hline
\end{tabular}

NA: refers to nothing. 


\section{References}

1. Dewey, K.G.; Begum, K. Long-term consequences of stunting in early life. Matern. Child Nutr. 2011, 7, 5-18. [CrossRef] [PubMed]

2. Black, R.E.; Victora, C.G.; Walker, S.P. Maternal and child undernutrition and overweight in low-income and middle-income countries. Lancet 2013, 382, 427-451. [CrossRef]

3. Martorell, R.; Zongrone, A. Intergenerational influences on child growth and undernutrition. Paediatr. Perinat. Epidemiol. 2012, 26, 302-314. [CrossRef] [PubMed]

4. Koletzko, B.; Brands, B.; Poston, L.; Godfrey, K.; Demmelmair, H. Early nutrition programming of long-term health. Proc. Nutr. Soc. 2012, 71, 371-378. [CrossRef] [PubMed]

5. Das, J.K.; Salam, R.A.; Imdad, A.; Bhutta, Z.A. Infant and Young Child Growth. In Reproductive, Maternal, Newborn, and Child Health: Disease Control Priorities, 3rd ed.; Black, R.E., Laxminarayan, R., Temmerman, M., Walker, N., Eds.; The International Bank for Reconstruction and Development/The World Bank(c): Washington, DC, USA, 2016; Volume 2.

6. Mangasaryan, N.; Martin, L.; Brownlee, A.; Ogunlade, A.; Rudert, C.; Cai, X. Breastfeeding promotion, support and protection: Review of six country programmes. Nutrients 2012, 4, 990-1014. [CrossRef] [PubMed]

7. Daelmans, B.; Ferguson, E.; Lutter, C.K. Designing appropriate complementary feeding recommendations: Tools for programmatic action. Matern. Child Nutr. 2013, 9, 116-130. [CrossRef] [PubMed]

8. Menon, P.; Rawat, R.; Ruel, M. Bringing rigor to evaluations of large-scale programs to improve infant and young child feeding and nutrition: The evaluation designs for the Alive \& Thrive initiative. Food Nutr. Bull. 2013, 34, S195-S211. [PubMed]

9. Dhingra, U.; Gittelsohn, J.; Suleiman, A.M. Delivery, immediate newborn and cord care practices in Pemba Tanzania: A qualitative study of community, hospital staff and community level care providers for knowledge, attitudes, belief systems and practices. BMC Pregnancy Childbirth 2014, 14, 173. [CrossRef] [PubMed]

10. World Health Organization; UNICEF. Strengthening Action to Improve Feeding of Infants and Young Children 6-23 Months of Age in Nutrition and Child Health Programmes; World Health Organization: Geneva, Switzerland, 2008.

11. World Health Organization. Indicators for Assessing Infant and Young Child Feeding Practices Part I: Definitions; WHO/World Health Organization: Geneva, Switzerland, 2008.

12. Ruel, M.T.; Brown, K.H.; Caulfield, L.E. Moving forward with complementary feeding: Indicators and research priorities. International Food Policy Research Institute (IFPRI) discussion paper 146 (April 2003). Food Nutr. Bull. 2003, 24, 289-290. [CrossRef] [PubMed]

13. Arimond, M.; Daelmans, B.; Dewey, K. Indicators for feeding practices in children. Lancet 2008, 371, 541-542. [CrossRef]

14. Roche, M.L.; Sako, B.; Osendarp, S.J.; Adish, A.A.; Tolossa, A.L. Community-based grain banks using local foods for improved infant and young child feeding in Ethiopia. Matern. Child Nutr. 2017, 13, e12219. [CrossRef] [PubMed]

15. Burns, J.; Emerson, J.A.; Amundson, K.; Doocy, S.; Caulfield, L.E.; Klemm, R.D. A Qualitative Analysis of Barriers and Facilitators to Optimal Breastfeeding and Complementary Feeding Practices in South Kivu, Democratic Republic of Congo. Food Nutr. Bull. 2016, 37, 119-131. [CrossRef] [PubMed]

16. Jones, A.D.; Cruz Agudo, Y.; Galway, L.; Bentley, J.; Pinstrup-Andersen, P. Heavy agricultural workloads and low crop diversity are strong barriers to improving child feeding practices in the Bolivian Andes. Soc. Sci. Med. 2012, 75, 1673-1684. [CrossRef] [PubMed]

17. Fabrizio, C.S.; van Liere, M.; Pelto, G. Identifying determinants of effective complementary feeding behaviour change interventions in developing countries. Matern. Child Nutr. 2014, 10, 575-592. [CrossRef] [PubMed]

18. Pelto, G.H.; Martin, S.L.; van Liere, M.J.; Fabrizio, C.S. Perspectives and reflections on the practice of behaviour change communication for infant and young child feeding. Matern. Child Nutr. 2015, 12, 245-261. [CrossRef] [PubMed]

19. Tong, A.; Flemming, K.; McInnes, E.; Oliver, S.; Craig, J. Enhancing transparency in reporting the synthesis of qualitative research: ENTREQ. BMC Med. Res. Methodol. 2012, 12, 181. [CrossRef] [PubMed] 
20. Moher, D.; Liberati, A.; Tetzlaff, J.; Altman, D.G. Preferred reporting items for systematic reviews and meta-analyses: The PRISMA statement. PLoS Med. 2009, 6, e1000097. [CrossRef] [PubMed]

21. The World Bank Group. Country and Lending Groups. 2016. Available online: http://data.worldbank.org/ about/country-and-lending-groups (accessed on 24 March 2016).

22. Critical Appraisal Skills Programme. CASP Qualitative Research Checklist. 2017. Available online: http: / / www.casp-uk.net/casp-tools-checklists (accessed on 12 November 2016).

23. Lewin, S.; Glenton, C.; Munthe-Kaas, H. Using qualitative evidence in decision making for health and social interventions: An approach to assess confidence in findings from qualitative evidence syntheses (GRADE-CERQual). PLoS Med. 2015, 12, e1001895. [CrossRef] [PubMed]

24. Thomas, J.; Harden, A. Methods for the thematic synthesis of qualitative research in systematic reviews. BMC Med. Res. Methodol. 2008, 8, 45. [CrossRef] [PubMed]

25. Lakshman, R.; Ogilvie, D.; Ong, K. Mothers' experiences of bottle-feeding: A systematic review of qualitative and quantitative studies. Arch. Dis. Child. 2009, 94, 596-601. [CrossRef] [PubMed]

26. Mazarello Paes, V.; Ong, K.; Lakshman, R. Factors influencing obesogenic dietary intake in young children (0-6 years): Systematic review of qualitative evidence. BMJ Open 2015, 5, e007396. [CrossRef] [PubMed]

27. McInnes, R.J.; Chambers, J.A. Supporting breastfeeding mothers: Qualitative synthesis. J. Adv. Nurs. 2008, 62,407-427. [CrossRef] [PubMed]

28. De Jager, E.; Skouteris, H.; Broadbent, J.; Amir, L.; Mellor, K. Psychosocial correlates of exclusive breastfeeding: A systematic review. Midwifery 2013, 29, 506-518. [CrossRef] [PubMed]

(C) 2017 by the authors. Licensee MDPI, Basel, Switzerland. This article is an open access article distributed under the terms and conditions of the Creative Commons Attribution (CC BY) license (http:/ / creativecommons.org/licenses/by/4.0/). 